evidence that sibutramine achieve this goal, and weight loss with sibutramine is associated with competing risks that have not been assessed long-term. Several RCT quality issues are discussed in detail, and we suggest a need for updated efficacy and safety reporting guidelines for in this area.

THE COMING EPIDEMIC OF OBESITY IN ELDERLY AMERICANS. D.E. Arterburn ${ }^{1}$, P.K. Crane ${ }^{2}$, S.D. Sullivan ${ }^{2} ;{ }^{1}$ VA Puget Sound Health Care System, Seattle, WA; ${ }^{2}$ University of Washington, Seattle, WA (Tracking ID \#76608)

BACKGROUND: The prevalence of obesity among the elderly is increasing. If this trend among the elderly continues, obesity may present new challenges to the health-care delivery and financing systems in the United States. No published models exist predicting future prevalence of obesity in the elderly.

METHODS: We examined changes in obesity prevalence by U.S. birth cohorts using data from five nationally-representative surveys, NHES; NHANES I, II, and III; and NHANES-C, and the US Census Bureau. Using these estimates, we calculated the prevalence of obesity (body mass index $(\mathrm{BMI})>30)$ and normal weight $(\mathrm{BMI}<25)$ among the elderly in 2010 under three different scenarios of obesity prevalence change (worst-case, best-case, and mid point scenario).

RESULTS: We estimate that the prevalence of obesity among adults over age 60 will increase from $32.0 \%$ in 2000 to $37.4 \%$ in 2010 (mid point scenario). Under the best-case scenario, the prevalence of obesity would still increase to $33.6 \%$, and under the worst-case it would reach $39.6 \%$. The number of obese elders will increase from 14.6 to 20.9 million (mid point scenario). Similarly, using our mid point scenario, we estimate that the prevalence of normal weight will decline from $38.2 \%$ in 2000 to $26.7 \%$ in 2010 among elderly individuals. We also present changes in obesity prevalence for four US birth cohorts over a forty year period. Our results were robust to sensitivity analyses that used alternate US Census population projections and estimates of prevalence change.

CONCLUSION: Given the rapid rise in obesity prevalence among middle-aged adults over the last 40 years in the United States, we predict that the proportions and especially the numbers of obese elderly individuals will continue to increase substantially. Since obesity is associated with increased risk of several chronic diseases, reduced quality of life, reduced functional status, increased health costs, and increased utilization, this trend may challenge the health care delivery and financing systems in the United States. Our work demonstrates the effect of birth cohorts on future obesity prevalence. Other published models used to estimate the effect of the aging baby-boom generation and increased longevity on future health care spending have failed to account for the changing prevalence of obesity and associated diseases. We conclude with a detailed discussion of the health and economic implications of this trend for elderly Americans.

\title{
SCIENTIFIC ABSTRACTS
}

\section{CHRONIC DISEASES E/CM}

THE EFFICACY AND SAFETY OF SIBUTRAMINE FOR WEIGHT LOSS: A SYSTEMATIC REVIEW. D.E. Arterburn ${ }^{1}$, P.K. Crane ${ }^{2}$, D.L. Veenstra ${ }^{2}$; ${ }^{1}$ VA Puget Sound Health Care System, Seattle, WA; ${ }^{2}$ University of Washington, Seattle, WA (Tracking ID \#76057)

BACKGROUND: Over 100 million adults are currently eligible to receive drug therapy for obesity in the United States, and this number is likely to continue to grow. Unfortunately, few drug treatment options for obesity exist. We conducted a systematic review to assess the efficacy and safety of sibutramine for weight loss.

METHODS: In April 2002, we searched MEDLINE, EMBASE, The Cochrane Library, and 7 orther computerized bibliographic search tools using the key words "sibutramine," "meridia," and "reductil", and identified 1245 citations. These citations were reviewed to identify randomized, placebo-controlled trials (RCT) of sibutramine 10-20 mg daily in adults. The methodological quality of each RCT was assessed. We identified 44 potentially relevant RCTs. Trials with complete data for weight loss and similar treatment duration (3 months, 6 months, and one year trials) were pooled for meta-analysis. Heterogeneity and publication bias were assessed. Authors of RCTs with incomplete data for abstraction were contacted and missing data was requested ( $70 \%$ response rate).

RESULTS: A total of 29 RCTs had sufficient data for analysis after including unpublished data from 11 contacted authors. Trials had varying durations of treatment ( 3 months, 6 months, one year, two-year weight maintenance trial). The weighted mean differences in weight loss, sibutramine minus placebo, for the 3 month and one year trials were $-2.8 \mathrm{~kg}(95 \% \mathrm{CI}:-2.3$ to $-3.3)$ and $-4.4 \mathrm{~kg}(95 \% \mathrm{CI}:-3.6$ to -5.3$)$, respectively. The 6 month trials were statistically heterogeneous, and evidence of publication bias was found. One trial found sibutramine maintains weight better than placebo at two years. One adverse effect was reported with enough consistency to allow pooling: sibutramine increases heart rate by $3.8 \mathrm{bpm}$ ( $95 \%$ CI 2.70 to 4.82). Due to heterogeneity and publication bias, sibutramine's effects on blood pressure, cholesterol, and glucose control could not be pooled. There is no evidence of heart valve injury at 6 months. Sibutramine's efficacy and safety have not been tested beyond two years. Average quality score of RCTs was high (3.2) on Jadad scale, however several additional quality issues were identified.

CONCLUSION: The accepted goal of obesity drug therapy is to reduce obesity-associated morbidity and mortality by improving cardiovascular and metabolic profiles. There is little
A METHOD TO IMPUTE MISSING DATE VALUES. A.M. Bayoumi ${ }^{1}$, C. Goia ${ }^{2} ;{ }^{1}$ Inner City Health Research Unit, St. Michael's Hospital, Toronto, Ontario; ${ }^{2}$ Ontario HIV Treatment Network, Toronto, Ontario (Tracking ID \#76505)

BACKGROUND: Although techniques to impute missing values have been elaborated for continuous and categorical data, similar methods for missing date values are not well established. Such methods may be particularly important for studies where several date values interact, such as the construction of antiretroviral drug regimens for human immunodeficiency virus (HIV) positive patients. We developed and evaluated a method of imputing missing date values for antiretroviral drug records.

METHODS: We studied participants in the HIV Ontario Observational Database, an observational longitudinal cohort study. Antiretroviral drug records were abstracted from medical chart review by trained data collectors. The year, month, and day of initiating an discontinuing antiretrovirals were recorded separately and each could be missing. Each record was characterized by the pattern of missing date values and potential correlates of missing values (drug class, study site, and timing of therapy initiation) were examined with multinomia logistic regression. We simulated a set of missing data by probabilistically dropping date elements from known records with complete data according to the predicted distribution from the regression equations and a randomly generated uninformative prior distribution. Next, we applied a set of midpoint imputation rules to the synthesized missing data, in which the earliest and latest possible dates for a missing value were calculated and averaged. We constructed antiretroviral drug regimens and compared results of the imputed data to the original data. RESULTS: We studied 16,954 antiretroviral drug records representing 2016 participants. Of these, 6859 records $(40.5 \%)$ had no missing values. The multinomial regression equations indicated that values were not missing at random but were associated with study site, drug class, and time of drug initiation. After simulating the missing dataset, 40 records were missing the start year or stop year for the drug record. All records $(n=370)$ for participants with such records were dropped. For drug start dates, drug stop dates, date of first antiretroviral use, date of first highly active antiretroviral therapy (HAART) use, and length of time interrupting therapy after starting therapy, the median differences between imputed and real values were all 0 . The corresponding 5 th and 95 th percentiles, in days, were $-7,8 ;-8,6 ;-6,8 ;-9,5 ;$ and 0,11 . Imputation resulted in incorrect classifications for $12(1.8 \%)$ of 664 participants who had never used HAART and $3(0.4 \%)$ of 830 who had ever used HAART.

CONCLUSION: Date imputation methods result in small deviations from real values in an observational database. When errors are introduced, they are usually small, even when imputation rules are used to make inferences about combination drug regimens.

THE RATIONAL CLINICAL EXAM: IS THIS PATIENT DEAD? $\underline{\text { C.M. Booth }}{ }^{1}$, R.H. Boone ${ }^{1}$ G. Tomlinson ${ }^{1}$, A.S. Detsky ${ }^{1}$; ${ }^{1}$ University of Toronto, Toronto, Ontario (Tracking ID \#75993) BACKGROUND: Most survivors of cardiac arrest are comatose after resuscitation and meaningful neurological recovery occurs in only a small proportion of cases. Treatment can be 
lengthy, expensive, and is often emotionally difficult for families and caregivers. Physical examination is potentially useful in this common clinical scenario, and the information obtained may help physicians and families making accurate decisions concerning treatment and/or withdrawal of care. The purpose of this review was to determine the precision and accuracy of the clinical examination in predicting poor outcome (death, vegetative state, or severe neurological impairment) in post-cardiac arrest coma.

METHODS: We conducted a search of MEDLINE for English-language articles published between 1966 and 2002 related to the precision and accuracy of the physical examination in comatose survivors of cardiac arrest. Studies were included if they assessed the precision and accuracy of the clinical examination in prognosis of post-cardiac arrest coma in adults. Eleven studies of the accuracy of the clinical exam met inclusion criteria and were included in this review. Two authors independently reviewed each study to determine eligibility, abstract data, and classify methodologic quality using pre-determined criteria. Disagreement was resolved by consensus.

RESULTS: Summary likelihood ratios (LRs) were calculated from random effects models. Five clinical signs were found to strongly predict death or poor neurological outcome: absent corneal reflexes at 24 hours (LR, 12.9; 95\% confidence interval [CI], 2.0-68.7), absent pupillary response at 24 hours (LR, 10.2; $95 \%$ CI, 1.8-48.6), absent withdrawal response to pain at 24 hours (LR, 4.7; 95\% CI, 2.2-9.8), no motor response at 24 hours (LR, 4.9; 95\% CI, 1.6-13.0), and no motor response at 72 hours (LR, 9.2; 95\% CI, 2.1-49.4). The proportion of individuals dying or having a poor neurological outcome was calculated by pooling the outcome data from the 11 studies $(n=1914)$ and used as an estimate of the pre-test probability of poor outcome. The random effects estimate of poor outcome was 77\% (95\% CI, 72-80\%). The best LR increases the pre-test probability of $77 \%$ to a post-test probability of $97 \%$ ( $95 \%$ CI $87-100 \%$ ). No clinical findings were found to have LRs which strongly predicted good neurological outcome.

CONCLUSION: Simple physical examination maneuvers strongly predict death or poor neurological outcome in comatose survivors of cardiac arrest. The most useful signs occur at 24 hours post-cardiac arrest, and earlier prognosis should not be made on the basis of the clinical examination alone.

BLOOD PRESSURE COMPONENTS AS RISK FACTORS FOR CARDIOVASCULAR DISEASE MORTALITY. T. Bowman ${ }^{1}$, H. Sesso ${ }^{2}$, M. Gaziano ${ }^{1} ;{ }^{1}$ Harvard University, Boston, MA; ${ }^{2}$ Brigham and Women's Hospital, Boston, MA (Tracking ID \#75702)

BACKGROUND: Both systolic blood pressure (SBP) and diastolic blood pressure (DBP) are risk factors for cardiovascular disease (CVD). It is unclear whether pulse pressure (PP) and mean arterial pressure (MAP) provide additional information in predicting CVD risk. We analyzed data from the Physicians' Health Study enrollment cohort to evaluate the relationship between SBP, DBP, PP, MAP and the risk of CVD mortality in men.

METHODS: In a prospective cohort design, 59,687 normotensive men free of CVD and cancer completed a baseline questionnaire. Data were collected on coronary risk factors including self-reported SBP, DBP, PP (SBP-DBP) and MAP (2/3SBP + 1/3DBP). Using the National Death Index, death certificates were obtained and ICD codes were used to identify cases of CVD death. Relative Risks (RRs) and 95\% confidence intervals were computed using Cox proportional hazards models adjusting for coronary risk factors.

RESULTS: During a mean follow-up of 5.5 years, 588 men (median age $=67.3$ ) died from CVD. At baseline, mean SBP was $125.0 \pm 11.3$, DBP was $78.0 \pm 7.0$, PP was $47.0 \pm 8.9$ and MAP was $93.7 \pm 7.6$. The corresponding multivariate RR's of CVD death for $10 \mathrm{~mm} \mathrm{Hg}$ increments of SBP, DBP, PP and MAP were 1.22 (1.14-1.30), 1.14 (1.01-1.29), 1.23 (1.14$1.33)$ and $1.28(1.14-1.42)$. We then considered the joint effects of combinations of $\mathrm{BP}$ measurements in the multivariate model (Table 1).

CONCLUSION: All four BP components were each independent predictors of CVD death. When considered in combination with any of the other three BP measures, SBP was the only BP measurement that consistently remained a significant predictor. This suggests that SBP may be the most informative blood pressure measurement in the prediction of CVD death among initially healthy normotensive men.

\section{Comparison of RR's in the combined multivariate models (10 mm Hg increments)}

$\begin{array}{lcccc} & \text { SBP } & \text { DBP } & \text { PP } & \text { MAP } \\ \text { Model \#1 } & 1.24(1.15-1.34) & 0.94(0.82-1.08) & & \\ \# 2 & 1.17(1.04-1.31) & & 1.06(0.93-1.22) & \\ \# 3 & 1.28(1.13-1.45) & & & 0.91(0.74-1.12) \\ \# 4 & & 1.17(1.04-1.31) & 1.24(1.15-1.34) & \\ \# 5 & & 0.61(0.47-0.79) & & 1.91(1.52-2.41) \\ \# 6 & & & 1.18(1.08-1.28) & 1.17(1.04-1.31)\end{array}$

COMPLIMENTARY OR ALTERNATIVE: DOES ALTERNATIVE MEDICINE USE DELAY CANCER THERAPY? C.L. Bryson ${ }^{1}$, G. Davis ${ }^{2}$, B. Yueh ${ }^{2}$, M.B. McDonell ${ }^{2}$, M. Micek ${ }^{2}$, S.D. Fihn ${ }^{2} ;{ }^{1}$ VA Puget Sound Health Care System, Seattle, WA; ${ }^{2}$ University of Washington, Seattle, WA (Tracking ID \#75523)

BACKGROUND: If complementary or alternative medicine (CAM) is used instead of, rather than in addition to traditional treatments, the latter might be delayed. Recent studies have shown $31 \%$ of cancer patients utilize a form of CAM. We explored whether CAM use reduced the likelihood of receiving traditional therapy.

METHODS: A CAM questionnaire was mailed to participants in the Ambulatory Care Quality Improvement Project, a randomized trial involving 7 VA outpatient centers. Subjects who returned a CAM questionnaire and were diagnosed with cancer during the last 1.5 years of the trial were studied. Diagnosis and treatment were both ascertained from computerized inpatient and outpatient records. Medicare data were available for those over 65. Hazard ratios for treatment (HR) were calculated from Cox models for those who received treatment more than 14 days after diagnosis. Subjects for whom no definitive treatment was observed were censored. RESULTS: There were 16,640 subjects who completed and returned a CAM questionnaire; $823(5 \%)$ developed incident cancer and fit inclusion criteria. Fifty percent $(n=408)$ of the subjects reported some use of CAM at any time in the past, with $241(30 \%)$ reporting use within the past year. There were 704 subjects who did not receive therapy in the first 14 days after diagnosis of cancer, with 160 definitive treatments observed before the end of the study. Those using CAM within the past year did not have a decrease in the likelihood of therapy (age adjusted HR $0.82,95 \%$ CI 0.6-1.2) compared with patients who never used CAM. Those who had ever used CAM were $35 \%$ less likely to receive traditional therapy (age adjusted HR $0.65,95 \%$ CI $0.5-0.9 ; \mathrm{p}=0.01$ ) compared with those who never used CAM therapy.

CONCLUSION: Ever using CAM but not current use was associated with a lower risk of receiving traditional therapy among veterans, suggesting users of CAM may be less likely to accept traditional therapy.

OUTPATIENT PHARMACY CARE AND HIV-1 RNA RESPONSE AMONG PATIENTS ON HIGHLY ACTIVE ANTIRETROVIRAL THERAPY. E. Castillo ${ }^{1}$, A. Palepu ${ }^{1}$, A. Beardsell ${ }^{2}$, B. Yip ${ }^{2}$, J.S. Montaner ${ }^{1}$, R. Hogg ${ }^{1}$; ${ }^{1}$ University of British Columbia, Vancouver, British Columbia; ${ }^{2}$ British Columbia Centre for Excellence in HIVIAIDS, St. Paul's Hospital, Vancouver, British Columbia (Tracking ID \#74423)

BACKGROUND: Highly active antiretroviral therapy (HAART) has been shown to prolong survival in persons with AIDS and those with intermediate-stage HIV-1 infection. Adherence to complex regimens is necessary to achieve long-term effectiveness. The impact of HIV/ AIDS-specific pharmacy services on patient adherence and HIV-1 RNA suppression is currently not well described. This study aimed to compare the impact of differing levels of HIV-pharmacy care on adherence and time to HIV-1 RNA suppression among participants on HAART enrolled in a population-based HIV/AIDS Drug Treatment program in British Columbia.

METHODS: Retrospective observational study of 788 treatment-naÿve patients who started HAART between August 1997 and July 2000 and were followed until March 31, 2002. The degree of outpatient pharmacy care was defined according to pharmacy dispensing site for the participants' first prescription of HAART: highest at the AIDS-tertiary care hospital outpatient pharmacies, intermediate at HIV/AIDS Drug Treatment Program funded off-site pharmacies and lowest at family physician's offices. Cox-proportional hazard models examined the independent effect of pharmacy dispensing site on time to two consecutive HIV-1 RNA suppressions controlling for other prognostic factors including physicians' experience, age, gender, injection drug use, use of therapy containing NNRTI vs PI, adherence $>=90 \%$, AIDS diagnosis at baseline, baseline CD4 cell count and HIV-1 RNA.

RESULTS: Median time on antiretrovirals was 28 months (IQR 14-38). There were 489 (62.1\%) participants obtained their medications from the AIDS-tertiary care outpatient pharmacies; $98(12.4 \%)$ from off-site pharmacies and 201 (25.5\%) from their physicians' offices. The proportion of patients exhibiting $>=90 \%$ adherence to treatment was observed to be higher among patients receiving their HAART at the AIDS-tertiary care pharmacies compared to off-site pharmacies and to physicians' offices $(70.4 \%$ vs. $59.2 \%$ vs. $55.7 \%$; $\mathrm{p}=0.0001$ ). At 12 months, cumulative suppression rate was higher for patients receiving their first HAART prescriptions from the AIDS-tertiary care pharmacy in comparison with those from the off-site pharmacy and with for those from physicians' offices $(74.6 \%$ vs. $59.4 \%$ vs. $60.0 \%, \mathrm{p}=0.001)$. After adjusting for other prognostic factors, subjects who were first dispensed medications from the AIDS-tertiary care pharmacy were 1.49 times (95\% CI: 1.20 1.85) more likely to be suppressed than those getting their medications from off-site pharmacies and physicians' offices.

CONCLUSION: Providing regular outpatient pharmacy care is independently associated with improved HIV-1 RNA response. Standardization of pharmacy practices for dispensing HAART may improve outcomes for patients who receive their HIV medications from other non-tertiary care pharmacy sites.

NATIONAL TRENDS IN OFFICE VISITS AND ANALGESIC PRESCRIPTIONS FOR MUSCULOSKELETAL PAIN: 1980 VS. 2000. M. Caudill-Slosberg ${ }^{1}$, L. Schwartz ${ }^{1}$, S. Woloshin'; ${ }^{1}$ VA Medical Center, White River Junction, VT (Tracking ID \#77166)

BACKGROUND: The pharmacologic treatment of pain has received increasing attention over the past decade, promoted by national guidelines, the "pain as the 5 th vital sign" campaign and direct-to-consumer advertising. We examined national trends in outpatient office visits and analgesic treatment for musculoskeletal pain, comparing data from 1980 and 2000.

METHODS: We analyzed the National Ambulatory Medical Care Survey (NAMCS) - a nationally representative survey of visits to office-based physicians - using data from 1980-81 ( $\mathrm{n}=89,000$ visits) and $1999-2000$ ( $\mathrm{n}=45,000$ visits). To focus on visits where analgesics are likely to be the primary treatment, we selected visits where the patient said their most important reason for seeing the doctor was pain in the back, upper or lower extremities, neck, headache or generalized pain. Visits were defined as acute if the pain was present for less than 3 months; otherwise visits were defined as chronic.

RESULTS: Overall, annual musculoskeletal pain visit rates changed little between 1980 and 2000: 220 vs. 250 visits per 1000 people. The proportion of visits where nonsteroidal antiinflammatory and opioid medications were prescribed increased:

\begin{tabular}{|l|c|c|c|c|}
\hline & \multicolumn{2}{|c|}{ NSAIDS } & \multicolumn{2}{c|}{ Opioids } \\
\hline & 1980 & 2000 & 1980 & 2000 \\
\hline & & & & \\
\hline Acute visits & $19 \%$ & $34 \%$ & $8 \%$ & $10 \%$ \\
\hline Chronic visits & $26 \%$ & $31 \%$ & $8 \%$ & $13 \%$ \\
\hline
\end{tabular}


The largest changes were in the use of prescription NSAIDs for acute pain and opioids for chronic pain. Use of higher potency opioids (e.g. hydrocodone, oxycodone, morphine) for chronic pain tripled from $3 \%$ to $9 \%$ of visits. In addition to opioids, tramadol, a new drug with opiate-like characteristics was prescribed at $3 \%$ of chronic pain visits in 2000.

CONCLUSION: In spite of the increased attention to pain treatment, there has not been a substantial increase in office visits for musculoskeletal pain complaints. The threshold for prescribing NSAIDS and opioids, however, has decreased.

HEART RATE RECOVERY AS A PREDICTOR OF CARDIAC AND ALL-CAUSE MORTALITY AMONG PATIENTS WITH TYPE 2 DIABETES MELLITUS. K.M. Chacko', J.A. Dixon ${ }^{1}$, T.A. Bauer ${ }^{1}$, R.A. Lundgren ${ }^{1}$, R.O. Estacio ${ }^{2}$; ${ }^{1}$ University of Colorado Health Sciences Center, Denver, CO; ${ }^{2}$ Denver Health and Hospital Authority, Denver, CO (Tracking ID \#75516)

BACKGROUND: Slowed heart rate recovery (HRR) following peak exercise has been shown to be an independent predictor of all-cause mortality in healthy adults as well as in patients with documented heart disease. To date, there are no studies specifically evaluating the relationship between HRR, cardiac outcomes, and mortality in patients with type 2 diabetes mellitus (T2DM). The current study examines this relationship over a 5 -year follow-up period. METHODS: Subjects consisted of T2DM patients enrolled in the Appropriate Blood Pressure Control in Diabetes trial. Charts were reviewed retrospectively and all patients who performed symptom-limited Bruce protocol exercise treadmill tests at baseline $(n=890)$ were included in the analysis. Utilizing existing literature, abnormal HRR at 1 minute was defined as a decrease of 12 beats per minute (bpm) or less. A chi-square test was used to evaluate the relationship between the published cutoff for abnormal HRR and both cardiovascular and all-cause mortality. Chronotropic incompetence (C.I.), defined as failure to acheive $85 \%$ of agepredicted peak maximal heart rate, was evaluated along with HRR in a mulitple logisitc regression model for their relationship to all-cause and cardiovascular mortality.

RESULTS: Of 871 patients with 1-minute HRR data, 188 had an abnormal HRR and 144 had C.I. All-cause mortality among patients with an abnormal HRR was $11.7 \%$ vs. $6.3 \%$ among patients with a normal HRR ( $\mathrm{p}=0.01$ ), and cardiovascular mortality was $5.9 \%$ vs. $3.5 \%$ in patients with an abnormal HRR versus a normal HRR respectively $(\mathrm{p}=0.15)$. Patients demonstrating C.I. had an OR for all-cause mortality of $2.28(95 \%$ CI $1.3-4.1, \mathrm{p}=0.006)$ and an OR for cardiovascular mortality of $3.0(95 \%$ CI $1.3-6.8, \mathrm{p}=0.009)$.

CONCLUSION: In the present study of patients with T2DM, HRR at 1 minute after maximal exercise was a significant independent predictor of all-cause mortality. HRR is less predictive of cardiovascular mortality in this group. These findings suggest that although there are higher event rates among those with HRR of less than $12 \mathrm{bpm}$, this specific cutoff may not be relevant for a population with T2DM. C.I. is a stronger predictor of both all-cause and cardiovascular mortality in this population.

DOES AMANTADINE IMPROVE THE EFFICACY OF INTERFERON IN INDIVIDUALS WITH CHRONIC HEPATITIS C? G. Chander ${ }^{1}$, K.A. Gebo ${ }^{1}$, S. Leoniak ${ }^{1}$, M. Jenckes ${ }^{1}$, M. Sulkowski ${ }^{1}$, S.N. Goodman ${ }^{1}$, E.B. Bass ${ }^{1}$; ${ }^{1}$ Johns Hopkins University, Baltimore, MD (Tracking ID \#76261)

BACKGROUND: As the prevalence of chronic hepatitis $\mathrm{C}$ rises, general internists will need to be increasingly familiar with alternate interferon-based combination therapies such as interferon (IFN) and amantadine. We performed a systematic review of the literature to assess the efficacy of the combination of amantadine and standard IFN compared to standard IFN alone in patients with chronic hepatitis C.

METHODS: We searched 8 electronic databases from January 1996 to March 2002. We identified additional studies by searching for references in pertinent studies, recent journals and by questioning experts. Studies were eligible for review if they were randomized controlled trials (RCT), reported original human data, and used virological or histological outcome measures. Meta-analysis was performed using the Mantel-Haenszel and Der Simonian and Laird Methods after checking for qualitative and statistical heterogeneity of the studies. To assess for possible publication bias we ran the analysis with and without studies reported only in abstracts.

RESULTS: Out of 30 RCTs in treatment naÿve individuals with chronic hepatitis C, 5 addressed the efficacy of IFN and amantadine, with a total of 888 patients. Mean age ranged between 38 and 48 years, and $26-71 \%$ of patients had hepatitis C genotype 1 . Study quality varied with only two trials being double-blinded and one study reporting on allocation concealment. The rate of sustained virological response (SVR) ranged from 10-29\% in the combination group, and from $14-22 \%$ in the control group. A meta-analysis of the 5 studies demonstrated a $4 \%$ absolute risk difference (95\% confidence interval (CI), $-; 3$ to $12 \%$ ) in SVR in treatment naïve individuals receiving standard IFN with amantadine compared to standard IFN monotherapy. Meta-analysis including an additional 4 abstracts demonstrated a $2 \%$ absolute risk difference $(95 \% \mathrm{CI},-4$ to $8 \%$ ). There was no significant statistical heterogeneity with either meta-analysis $(\mathrm{p}>0.05)$. In 5 studies of IFN and amantadine in previous IFN nonresponders, standard IFN with amantadine was no better than either standard IFN alone or IFN and ribavirin in achieving an SVR, but triple therapy with standard IFN, amantadine, and ribavirin achieved an increase in SVR in one small study $(\mathrm{N}=60)$. These studies were not pooled secondary to qualitative heterogeneity.

CONCLUSION: We conclude that amantadine adds little to the efficacy of standard interferon in the treatment of chronic hepatitis C.

PSYCHOSOCIAL FACTORS AND QUALITY OF LIFE IN CHRONIC DIALYSIS PATIENTS IN URBANIZED SOUTHERN INDIA. A. Chandrakantan ${ }^{1}$, A. Chandrakantan ${ }^{2}$, G. Abraham ${ }^{3}$; ${ }^{1}$ Dartmouth Hitchcock Medical Center, Lebanon, $\mathrm{NH} ;{ }^{2}$ University of Alabama at Birmingham, Birmingham, AL; ${ }^{3}$ Sri Ramachandra Medical College and Research Institute, Chennai, Tamilnadu (Tracking ID \#73604)

BACKGROUND: End-stage renal disease (ESRD) has had a considerable impact on the functional status and quality of life (QoL) as perceived by patients and has been the subject of intensive research for more than 2 decades. The purpose of this study was to examine the effect of psychosocial factors on a population with ESRD from all socioeconomic strata in an urban setting from Chennai, India.

METHODS: The study was based upon a survey consisting of about 100 questions, spanning numerous aspects of the patient's day-to-day living including chronic pain, sexual function, sleep disturbances, and cognitive function. The questions were based upon modifications of numerous available kidney quality of life dialysis instruments, cognitive function indices, and sleep assessment instruments.

RESULTS: There were 35 males and 18 females in the study. The mean age of the patients was 49.5 years. The mean time on hemodialysis was 32.8 months. In their overall health rating, on a scale of 1 (worst) to 10 (best), the patients mean score was 3.63 ; the mean rating compared to one year ago was slightly better at 4.09. Dialysis related issues: The most common complaints included fatigue, lack of strength, excessive thirst, dry mouth, and sleeping trouble. $19 \%$ of patients reported difficulty complying with the food restrictions, however only $9 \%$ reported difficulty with fluid restrictions. Psychosocial issues: Many patients reported significant social issues; $62.2 \%$ reported difficulty enjoying time with family, $20.7 \%$ reported difficulty with traveling on a day to day basis, and $83 \%$ reported difficulty lifting heavy weights they were able to lift before. $28 \%$ reported having decreased perception about their personal appearance, $38 \%$ reported a lack of time due to thrice weekly dialysis, $68 \%$ reported difficulties being able to squat to perform their ADL's. Up to $50 \%$ of patients reported mild to moderate cognitive impairment, and several males in the study reported sexual dysfunction. Up to $40 \%$ of the patients reported significant sleep disturbances specifically an inability to fall asleep or being awoken in the middle of their sleep. Greater than $50 \%$ of patients reported feeling motivated and informed about dialysis.

CONCLUSION: The incidence of depression, sleep disturbances, and sexual dysfunction are both under-recognized and under-treated in chronic hemodialysis patients, regardless of the care setting.The expectations of the standards of care will increase, as our knowledge of the psychosocial implications of chronic disease continue to be elucidated, and more data becomes available.

ASSOCIATION BETWEEN ALCOHOL CONSUMPTION AND DIABETES CARE-DATA FROM THE 2001 BEHAVIORAL RISK FACTOR AND SURVEILLANCE SYSTEM (BRFSS). L.D. Chew ${ }^{1}$, K.M. Nelson ${ }^{1}$, K.A. Bradley ${ }^{1}$; ${ }^{1}$ VA Puget Sound Health Care System, University of Washington, Seattle, WA (Tracking ID \#76871)

BACKGROUND: Although excessive alcohol intake may alter patient behavior and thus affect patient compliance, little is known about the effect of alcohol consumption on diabetes selfcare. We sought to determine the association between patient reports of alcohol consumption and diabetes care quality and adherence to diabetes self-management.

METHODS: We analyzed data from a nationally representative population-based survey, the 2001 Behavior Risk Factor and Surveillance System (BRFSS). We included 5322 adults (age>17) with a reported diagnosis of diabetes. Based on self-reported average alcohol consumption in the past 30 days, we categorized participants into the following groups: abstainers (none), low-level drinkers (drinks/day: $>1$ for women; $>2$ for men), and hazardous drinkers (drinks/day: $>1$ for women; $>2$ for men). A diabetes-specific BRFSS module was used to obtain clinical characteristics and preventive care practices from respondents with diabetes. Main analyses compared hazardous drinkers to all other diabetics (abstainers and low-level drinkers).

RESULTS: Two percent $(\mathrm{n}=95)$ of individuals with diabetes reported hazardous alcohol consumption, $29 \%$ reported low-level alcohol consumption, and $69 \%$ were abstainers. Hazardous drinkers were more likely to be male and employed compared to low-level drinkers or abstainers $(\mathrm{p}<0.05)$. Current smoking was more common among hazardous drinkers compared to abstainers and low-level drinkers $(40.3 \%$ vs. $16.0 \%, \mathrm{p}<0.001)$. Hazardous drinkers were more likely than other diabetics to report infrequent glucose self-monitoring (less often than weekly) $(29 \%$ vs. $17 \%, \mathrm{p}=0.03)$ and lack of participation in a diabetes education class ( $64 \%$ vs. $46 \%, \mathrm{p}=0.04)$. Hemoglobin A1C monitoring, annual foot and eye examinations, and self-foot examinations varied little across alcohol consumption categories. Hazardous drinkers were more likely than other diabetics to report infrequent glucose selfmonitoring $(\mathrm{OR}=2.4,95 \% \mathrm{CI}: 1.1-5.0)$ and having not participated in a diabetes education class $(\mathrm{OR}=2.3,95 \% \mathrm{CI}: 1.0-5.5)$, after controlling for age, gender, education, income, race, health care coverage, years since diabetes diagnosis, and insulin use.

CONCLUSION: Hazardous alcohol consumption among diabetic patients may be associated with poorer adherence to diabetes self-management, particularly glucose self-monitoring and participation in diabetes education. Primary care providers should consider screening for hazardous alcohol consumption to identify patients at risk for non-adherence to some diabetes self-care behaviors.

ASSOCIATION OF PHYSICAL AND SEXUAL ABUSE WITH HIGH-RISK SEXUAL BEHAVIOR IN HIV-INFECTED PERSONS WITH ALCOHOL PROBLEMS. C.H. Chuang ${ }^{1}$, J.M. Liebschutz ${ }^{1}$, N.J. Horton ${ }^{1}$, J.H. Samet ${ }^{1}$; ${ }^{1}$ Boston University, Boston, MA (Tracking ID \#73695)

BACKGROUND: Identifying individuals most at risk for transmitting HIV requires recognizing factors that predict high-risk sexual behavior among HIV-infected persons. We examined whether a history of physical or sexual abuse was associated with current high-risk sexual behavior among a cohort of HIV-infected persons with alcohol problems.

METHODS: The HIV-Alcohol Longitudinal Cohort (HIV-ALC) study recruited 349 HIVinfected subjects with a history of alcohol problems. At study entry, in-person interviews assessed physical and sexual abuse histories and sexual behaviors. Current high-risk sexual behavior was defined as having more than one sexual partner, exchanging sex for money or drugs, or not using condoms all the time in the past 6 months. Multivariable analyses assessed the relationship between histories of physical and sexual abuse and current high-risk sexual behavior controlling for sex, age, race, education, marital status, homelessness, depression, mode of HIV transmission, current alcohol, cocaine, heroin, and antiretroviral use. 
RESULTS: Among all subjects (20\% women), $81 \%$ reported a history of physical or sexual abuse during their lifetime. Lifetime sexual abuse (with or without physical abuse) was reported by $40 \%$, childhood sexual abuse (prior to age 13 ) by $26 \%$, and lifetime physical abuse (without sexual abuse) by $40 \%$. The prevalence of current high-risk sexual behavior was $51 \%$. In multivariable analyses, a history of lifetime sexual abuse (AOR $=1.97,95 \%$ CI $1.00-3.91$ ) was associated with current high-risk sexual behavior compared with subjects without any history of physical or sexual abuse. A history of childhood sexual abuse (AOR 1.85, 95\% CI 1.07-3.22) was also associated with current high-risk sexual behavior compared with subjects without a history of childhood sexual abuse.

CONCLUSION: The majority of HIV-infected persons with a history of alcohol problems reported high-risk sexual behaviors. In this cohort, even after controlling for other known factors associated with high-risk sexual behaviors, HIV-infected individuals with a history of lifetime sexual abuse and childhood sexual abuse were more at risk for engaging in high-risk sexual behaviors.

HEALTH RELATED QUALITY OF LIFE IN A RACIALLY DIVERSE PRIMARY CARE POPULATION. T.C. Collins ${ }^{1}$, M.E. Suarez-Almazor ${ }^{1}$, N.J. Petersen ${ }^{1} ;{ }^{1}$ Baylor College of Medicine, Houston, TX (Tracking ID \#75488)

BACKGROUND: We seek to compare the mean scores for each of the Medical Outcomes Study Short Form Survey (SF-36) components, stratified by race, to the standard norms for a population that closely parallels our cohort (i.e., patients with diabetes type II).

METHODS: Among a cohort of patients 50 years and older who participated in a screening study for peripheral arterial disease (PAD), we obtained information on sociodemographics and health related quality of life (SF-36). Patients were recruited from the Houston VA Medical Center and the Harris County Hospital District (Houston, TX).

RESULTS: Among 403 patients (136 Non-Hispanic Whites, 136 African Americans, and 131 Hispanics, 81 of whom were Spanish speaking), the mean age was $63.8 \pm .36$ years. PAD was present in $16.6 \%$ of patients. For the standard population, the range of mean scores was $41.52 \pm 11.27$ (physical component summary) to $82.04 \pm 24.96$ (social functioning). Within our cohort, the mean scores for Whites for each of the components ranged from $35.88^{-11.83}$ (physical component summary) to $72.25 \pm 23.63$ (mental health). With the exception of general health, role-emotional, and mental component summary, which did not differ statistically between the two groups, all other component means were statistically significant at $\mathrm{P}<.05$ with the population with diabetes type II having better scores. For African Americans within our

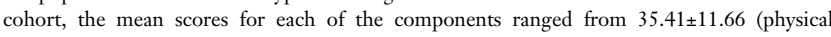
component summary) to $71.47 \pm 20.65$ (mental health); these lower means were all statistically significant at $\mathrm{P}<.01$. For Latinos within our cohort, the mean scores for each of the components ranged from $38.16 \pm 10.69$ (physical component summary) to $69.75 \pm 26.26$ (social functioning). For Latinos within our cohort, all means for each of the components were statistically significant, except vitality, at $\mathrm{P}<.001$.

CONCLUSION: When compared to a population of patients with diabetes type II, our racially diverse primary care clinic population had lower health related quality of life scores. The lower health related quality of life did not appear to differ greatly by race. Reasons for the poorer quality of life in our population may relate to unique characteristics of the patients screened including undefined social burdens or dissatisfaction with available health care. Further research is needed to define the resources needed within a primary care clinic visit to improve patients' health related quality of life.

VALIDATION OF THE WALKING IMPAIRMENT QUESTIONNAIRE IN AN ETHNICALLY DIVERSE PATIENT POPULATION. T.C. Collins ${ }^{1}$, M.E. Suarez-Almazor ${ }^{1}$, N.J. Petersen ${ }^{1}$, K. O'Malley'; ' ${ }^{1}$ Baylor College of Medicine, Houston, TX (Tracking ID \#75493)

BACKGROUND: Walking impairment is a common manifestation of peripheral arterial disease (PAD). In this study we present evidence for the validity of our Spanish translation of the Walking Impairment Questionnaire (WIQ).

METHODS: We screened patients $>=50$ years for PAD who were receiving care in primary care clinics in Houston, Texas. PAD was diagnosed by the ankle-brachial index (ABI; a level< 0.9 defined disease). Patients completed the Spanish versions of the San Diego Claudication Questionnaire, the WIQ, and the Medical Ouctomes Study Short Form Survey (SF-36) which provided information on claudication, walking ability (distance, speed, and stair climbing), and health related quality of life, respectively. Evidence for convergent and discriminant validity of the WIQ was obtained from correlations between the WIQ and other measures in both nonSpanish and Spanish speaking patients.

RESULTS: Among 403 patients (136 Non-Hispanic Whites, 136 African Americans, and 131 Hispanics, 81 of whom were Spanish speaking), the prevalence of PAD was $16.6 \%$. Convergent validity evidence was strong for both non-Spanish speaking and Spanish speaking patients; the correlations between the WIQ subscales and the SF-36 physical health measures were the strongest ranging from 0.36 to 0.66 ; all were significant at $\mathrm{P}<.01$. For Spanish speaking patients, the correlation between walking distance and physical health measures ranged from 0.44 to 0.74 ( $\mathrm{P}<.01$ for all correlations). For both groups the correlations of walking speed and stair climbing with all physical health measures had similar values and ranged from 0.36 to 0.72 ( $\mathrm{P}<.01$ for all correlations). The correlations of walking distance, speed, and stair climbing with emotional health measures of the SF-36 ranged from 0.29 to 0.49 for non-Spanish speaking patients ( $\mathrm{P}<.01$ for all correlations). For Spanish speaking patients, the correlations of walking ability with emotional health measures ranged from 0.18 to 0.44 ; all were statistically significant at $\mathrm{P}<.05$.

CONCLUSION: The WIQ scores correlated well with SF-36 components for both nonSpanish speaking and Spanish speaking patients. The strongest correlations were between the WIQ and the SF-36 physical health measures. Our findings suggest that our translation process did not limit our ability to capture valid data. Further research is needed to determine what specific items within the WIQ or the SF-36 questionnaire warrant restructuring to increase their validity for use in diverse populations.
SENSITIVITY AND SPECIFICITY OF METHODS APPLIED TO CLINICAL INFORMATION SYSTEMS FOR IDENTIFYING HIV-INFECTED PATIENTS WITH DIABETES. H. Crane $^{1}$, M.M. Kitahata'; ${ }^{1}$ University of Washington, Seattle, WA (Tracking ID \#75935)

BACKGROUND: Metabolic conditions are increasingly common among HIV-infected patients in the era of potent antiretroviral therapy, but the prevalence of treatment complications such as diabetes mellitus (DM) is unknown. Computerized clinical data captured in information systems could provide a useful tool for identifying specific conditions within a population.

METHODS: We examined four methods applied to computerized clinical data captured for a cohort of HIV-infected patients from 1995 to 2002 for identifying potential cases of type 2 DM: 1) billing and problem list data provided diabetes-related diagnoses (ICD-9 codes), 2) pharmacy data captured all medications used to treat DM (meds), and laboratory data was used to identify patients with 3) HbA1c $>7 \mathrm{mg} / \mathrm{dL}$ or 4) two random blood glucoses $>200 \mathrm{mg} /$ dL. Chart review was conducted to verify diagnoses of type 2 DM (gold standard) and exclude cases of type $1 \mathrm{DM}$.

RESULTS: 86 cases of type 2 DM were identified among a population of approximately 1600 $\mathrm{HIV}$-infected patients. Because the prevalence of DM among HIV-infected patients is low, small decreases in specificity resulted in substantial decreases in PPV. Used alone, ICD-9 diagnosis codes provided the highest level of sensitivity. Requiring that a patient have both an ICD-9 code and a diabetic medication resulted in the the highest specificity and PPV, but significantly lowered the sensitivity of the method.

CONCLUSION: Computerized data from clinical information systems can be used to identify HIV-infected patients with type 2 DM. The optimal method for identifying DM cases for a particular study will depend on the goals of the selection procedure.

Sensitivity, Specificity, and Positive Predictive Value of DM Case-Identification Techniques

$\begin{array}{lcc} & \text { Sensitivity } & \text { Specificity } \\ \text { ICD-9 Codes } & 0.92 & 0.99 \\ \text { Meds } & 0.76 & 0.99 \\ \text { HbA1c } & 0.62 & 1 \\ \text { Random gluc } & 0.66 & 0.97 \\ \text { ICD-9 Codes \& Meds } & 0.71 & 1 \\ \text { ICD-9 Codes Or Meds } & 0.98 & 0.98\end{array}$

PPV

0.82

0.78

0.54

ICD-9 Codes Or Meds

0.98

0.98

0.71

EFFECTIVENESS OF FOUR POPULAR DIETS FOR WEIGHT LOSS AND CARDIAC RISK REDUCTION: A RANDOMIZED CLINICAL TRIAL. M. Dansinger ${ }^{1}$, J. Augustin ${ }^{1}$, J. Griffith ${ }^{1}$, H.P. Selker ${ }^{1}$, E. Schaefer ${ }^{1} ;{ }^{1}$ Tufts-New England Medical Center, Boston, MA (Tracking ID \#77139)

BACKGROUND: Controversy surrounds the growing number of popular diet plans targeting weight loss and cardiac risk reduction. Data on the relative effectiveness of these strategies are limited, especially under conditions of realistic dietary adherence. We conducted a randomized clinical trial evaluating the effectiveness of 4 popular diet strategies for weight loss and cardiac risk reduction.

METHODS: One-hundred sixty adults with excess body mass and one or more metabolic cardiac risk factor were randomized to 1 of 4 dietary treatment strategies restricting either total fat (Ornish diet), total carbohydrates (Atkins diet), saturated fat and insulinogenic carbohydrates (Zone diet), or total calories (Weight Watchers diet). During the first 2 months of the study, subjects participated in 4 one-hour group sessions during which a dietitian and physician reviewed the rationale, recipes, meal plans and supplemental reading materials appropriate for the assigned strategy. After 2 months of intensive intervention aiming for maximum adherence, participants were asked to determine their own level of diet plan adherence for the following 10 months. Primary outcomes were changes in body mass index and Framingham cardiac risk score at 12 months. Secondary outcomes were changes in blood pressure, fasting serum lipids, glucose, glycohemoglobin, insulin, urine C-peptide, and renal function. Dietary adherence was assessed by 3-day diet diaries, urine nitrogen and ketones, and monthly telephone surveys. Data collection was completed in January 2003.

RESULTS: All 160 participants were recruited from the Greater Boston area. At baseline, median age was 50 years (range 23 to 73 ), $75 \%$ were Caucasian, $50 \%$ were male, $94 \%$ had dyslipidemia, $46 \%$ had elevated blood pressure, $47 \%$ had hyperglycemia or type II diabetes mellitus, and 6\% had known coronary artery disease. Mean body mass index was 34.7 (SD 3.9) $\mathrm{kg} / \mathrm{m}^{2}$, and mean Framingham score for 10-year risk of a coronary event was 9.1\% (SD 7.4\%). Diet records at baseline were consistent with the average American diet, with a mean daily intake of 2059 calories; $46 \%$ from carbohydrates, $35 \%$ from total fat, $12 \%$ from saturated fat, and $18 \%$ from protein. Final results for weight loss and cardiac risk factor reduction will be available at the time of the SGIM conference.

CONCLUSION: This randomized trial will provide much needed effectiveness data regarding popular diet strategies. It evaluates a broader range of popular diets and a longer follow-up period than any previously reported study. The results will have the potential to substantially validate or challenge conventional and alternative dietary approaches to cardiac risk and body fat reduction.

PROGNOSTIC SIGNIFICANCE OF PEAK OXYGEN CONSUMPTION MEASUREMENT DURING SYMPTOM LIMITED STRESS TESTING IN CORONARY DISEASE. ${ }^{\text {A. Devarajan }}{ }^{1}$, S. Pammal ${ }^{2}$, N. Maddukuri ${ }^{1}$; ${ }^{1}$ Jersey City Medical Center, New Jersey, NJ; ${ }^{2}$ Richland Cardiology and Internal Medicine, Mansfield, OH (Tracking ID \#74208)

BACKGROUND: Can a Peak oxygen consumption measurement during symptom limited stress testing be an independent predictor of mortality and morbidity in a stable coronary patient? 
METHODS: 164 consecutive coronary patients for cardiac evaluation had undergone symptom-limited stress testing using standard ramp protocol and simultaneous peak oxygen uptake was measured using Medgraphics CPX machine in an office setting

RESULTS: Patients in this study were followed up prospectively for an average period of 3 years. Of the 164 patients, 9 patients were lost for follow up. 18 patients were found to have VO2 (Oxygen uptake) of $14 \mathrm{ml} / \mathrm{kg} / \mathrm{min}$ or less out of which 6 expired, 3 refused intervention, and 9 had underwent either percutaneous transluminous coronary angioplasty or coronary artery bypass graft. Of the remaining 137 patients, only 6 needed Angioplasty, 3 expired due to various causes, 3 patients needed coronary bypass graft in the following three years.

Various studies have shown that peak oxygen uptake is an independent predictor of cardiac and all cause mortality in patients waiting for cardiac transplant. VO2 has a close linear relationship with cardiac output, which in turn to myocardial damage and or ischemia. Peak oxygen consumption can be safely measured in an office setting in stable coronary artery disease patients. There is more than $80 \%$ chance of MACE (Major adverse cardiac event) in patients who only reach peak VO2 of less than $14 \mathrm{ml} / \mathrm{kg} / \mathrm{min}$. Cardiac and all cause mortality and morbidity is significantly worse in patients in whom $\mathrm{VO} 2$ is less than $14 \mathrm{ml} / \mathrm{kg} /$ minute.

CONCLUSION: Measurement of Peak VO2 during symptom limited exercise stress testing can be used as an independent prognostic indicator for long-term morbidity and mortality in coronary disease.

PREVALENCE OF HYPOCHONDRIACAL WORRY IN A NATIONAL SAMPLE. C.C. Doebbeling ${ }^{1}$, L. Jones ${ }^{1}$, D. Langbehn ${ }^{1}$, R. Noyes ${ }^{1} ;{ }^{1}$ University of lowa Carver College of Medicine, lowa City, IA (Tracking ID \#76158)

BACKGROUND: Hypochondriacal worry (HC) is characterized by medically unexplained physical symptoms, bodily preoccupation, disease phobia, and the conviction that one has a disease despite reassurance. Significant worry leads to distress, impairment in functioning, and increased primary care utilization. Although the prevalence of illness worry has been studied in limited primary care samples, the national prevalence of this condition is not known. We sought to determine the prevalence and risk factors for HC in a national sample.

METHODS: Trained interviewers using a computer-assisted telephone interviewing system conducted surveys of individuals aged 18-64 years residing in the continental United States. Subjects were screened with the question "During the past 12 months, have you had a period of one month or more when you worried most of the time about having a serious illness?" The survey included items assessing demographics, doctor-confirmed medical conditions, psychiatric co-morbidity, domains of illness worry and childhood traumatic events. An equal sized control group was randomly selected from individuals who did not meet screening criterion. Descriptive and univariate statistics were calculated to compare factors associated with HC.

RESULTS: The overall response rate was $55 \%$. One-year prevalence of illness worry was $7 \%$. 123 subjects met criteria for HC. Compared to controls $(n=123)$, persons with HC were significantly more likely female; separated, divorced, or widowed; of low income; to have medical illness; to rank their current health as "fair" or "poor"; and to have psychiatric comorbidity including depression ( 22 vs. $6 \%$ ), generalized anxiety (17 vs. $6 \%$ ), and panic disorder (22 vs. $4 \%$ ). Persons with HC commonly saw a doctor about the worry $(81 \%)$, were distressed (71\%), and significant impairment (54\%), and had little or no reassurance (20\%). Childhood risk factors significantly differed between the groups. Those with HC were more likely to have experienced an illness or injury in a close friend or family member as traumatic, have a parent or family member addicted to alcohol or drugs, to have been a victim of violence, and to have been extremely ill or injured during childhood.

CONCLUSION: HC is prevalent among the American population and leads to impairment. Worriers are likely to access health care, have distress, have medical and psychiatric comorbidity, and report interference with their activities. The occurrence of illness or injury in self or loved ones, and experience with violence are important risk factors for illness worry. The general internist should consider screening for $\mathrm{HC}$ in vulnerable patients with high utilization.

HYPOCHONDRIACAL WORRY IN PERSONS WITH AND WITHOUT MEDICAL ILLNESS. C.C. Doebbeling ${ }^{1}$, L. Jones ${ }^{1}$, D. Langbehn ${ }^{1}$, R. Noyes ${ }^{1} ;{ }^{1}$ University of lowa Carver College of Medicine, lowa City, IA (Tracking ID \#76307)

BACKGROUND: Hypochondriacal worry (HC) is characterized by medically unexplained physical symptoms, bodily preoccupation, disease phobia, and the conviction that one has a disease despite reassurance. Significant worry leads to distress, impairment in functioning, and increased primary care utilization. The objective of this study was to compare persons with HC who had doctor-confirmed medical illness to those with $\mathrm{HC}$ who did not have illness.

METHODS: Trained interviewers using a computer-assisted telephone interviewing system conducted surveys of individuals aged 18-64 years residing in the continental United States. Subjects were screened with the question "During the past 12 months, have you had a period of one month or more when you worried most of the time about having a serious illness?" The survey included items assessing demographics, medical conditions, psychiatric comorbidity, domains of illness worry and medical utilization. Univariate and multivariate statistics were calculated to compare factors associated with illness worry.

RESULTS: The overall response rate was 55\%. 123 subjects met the screening criterion for HC. The one-year prevalence was $7 \%$. Most $(81 \%)$ subjects sought medical care for their worry, with $54 \%(n=67)$ reporting that a doctor had confirmed the presence of the illness of concern; $46 \%(n=56)$ did not have the illness they were worried about. Differences were found between those with the illness they worried about and those without. Those with the illness were older, had more depression, a longer period of worry, and more severe worry. Worriers with illness more frequently told doctors about their concern ( $81 \%$ vs. $66 \%)$, and had "little" or "not at all" reassurance ( $28 \%$ vs. $9 \%)$. Both groups reported seeing a doctor regarding the feared illness ( $90 \%$ ), "some" or "a lot" of distress (76\% and 64\%), and "some" or "a lot" of interference with activities (67\% and 38\%). Worriers without doctor-confirmed illness were more likely to worry about cancer $(55 \%$ vs. $16 \%)$. Worriers with illness had significantly greater medical utilization than those without. During the last year, they were more likely to have had doctor visits ( 16 vs. 6 visits), seen more doctors ( 4 vs. 2 doctors), had more hospital days ( 3 vs. 0.6 days), and taken medications ( 7 vs. 2 meds). Multivariate modeling did not reveal risk factor differences for $\mathrm{HC}$ between the two groups.

CONCLUSION: HC is prevalent among persons with and without medical illness. Worriers with medical illness, however, have greater distress, are less likely to be reassured, and have higher healthcare utilization. The general internist may wish to screen for HC in patients with medical illness who have high medical utilization.

ADJUSTMENT DISORDERS AMONG URBAN AND RURAL PRIMARY CARE PATIENTS IN AN INUSRED POPULATION. C.C. Doebbeling ${ }^{1}$, L.E. Jones ${ }^{1}$, R.F. Woolson ${ }^{2}$, B.N. Doebbeling ${ }^{1}$; ${ }^{1}$ University of lowa Carver College of Medicine, lowa City, IA; ${ }^{2}$ Medical University of South Carolina, Charleston, SC (Tracking ID \#76843)

BACKGROUND: Adjustment disorders (AD) are commonly diagnosed in medically ill patients. $\mathrm{AD}$ continue to receive little attention in the literature despite an estimated incidence of $5-21 \%$ in psychiatric consultation services for medically ill patients. The objective of this research was to describe the epidemiology and service use among primary care patients with $\mathrm{AD}$. METHODS: This is a retrospective cohort study of Wellmark Blue Cross/Blue Shield of Iowa enrollees who filed at least one claim for medical care during 1996-2001. Persons with initial mental health claims for AD (ICD-9 codes 308.00-308.20, 308.40-309.80, 309.82-309.90) were selected. Urban and rural designations were made using the metropolitan statistical area definition. Primary care providers (PCPs) included general internal medicine, general practice, or family practice physicians.

RESULTS: 942,808 individuals residing in Iowa were available for analysis. 211,001 (22\%) patients filed claims for mental disorders. AD accounted for more than $14 \%$ (30,215 patients) of all first mental health claims. Urban and rural subjects had a similar likelihood of receiving this diagnosis. Among rural dwellers, $1 / 3$ of all $\mathrm{AD}$ diagnoses were made by PCPs (33\% rural vs. $25 \%$ urban). Urban dwellers were more likely than rural dwellers to be hospitalized for the $\mathrm{AD}(0.25$ vs. 0.16 hospitalizations, $\mathrm{P}<0.0001)$, have more mental health visits ( 7.5 vs. 5.6 visits, $\mathrm{P}<0.0001$ ), and receive psychotherapy ( $38 \%$ vs. $34 \%, \mathrm{p}<0.0001) .39 \%$ of all $\mathrm{AD}$ patients later filed a claim for another mental health diagnosis, most commonly a mood disorder (50\%), anxiety disorder $(19 \%)$, or substance disorder $(8 \%)$. A small proportion of patients was referred to mental health providers (MHPs) (20\% urban; $15 \%$ rural) and saw MHPs a median of three months after initial $\mathrm{AD}$ diagnosis. In half of these cases, the diagnosis remained the same, over $1 / 3$ rd were changed to mood disorders, and $7 \%$ to anxiety disorders.

CONCLUSION: PCPs frequently diagnose AD. Although AD rarely resulted in hospitalization, outpatient utilization was substantial. Urban patients were more likely to receive mental health services. Mood and anxiety disorders accounted for the majority of follow-up diagnoses. More research should be directed at $\mathrm{AD}$ in primary care populations.

"TIRED BUT HAPPY": IDENTIFYING POSITIVE ASPECTS OF FATIGUE. M.B. Duke ${ }^{1}$, J.F. Wilson ${ }^{1}$, M. Andrykowski ${ }^{1}$, R. Lightner ${ }^{1}$; ${ }^{1}$ University of Kentucky, Lexington, KY (Tracking ID \#75867)

BACKGROUND: Fatigue, a symptom highly prevalent in clinic and population-based studies, is difficult to measure. Much of this difficulty stems from the multidimensional nature of fatigue, which may be described as a feeling state and by using neurocognitive parameters as well as physiologic measures. In a community-based survey, we previously determined that respondents could differentiate among physical, mental and emotional fatigue, and that each of these had a negative effect on quality of life. We wanted to determine if fatigue might also have a positive component. That is, could one be "tired but happy" and did this improve quality of life? METHODS: Using a random-digit dialing, statewide, telephone survey, we administered the MOS-20 measure of health related quality of life indicators to 661 adults. Respondents were asked how often they had experienced physical, mental and emotional fatigue during the previous one month. They were also asked about the experience of being "tired but happy". Quality of life measures were subscales from the MOS-20: physical, social and role functioning, mental health, health outlook and pain. Open-ended comments were solicited.

RESULTS: Among our respondents physical, mental, and emotional fatigue occurred in frequencies that followed a normal distribution, but the symptom of being "tired but happy" did not. Approximately $10 \%$ of respondents denied ever feeling "tired but happy". The remainder were evenly divided in reporting this symptom, with approximately $20 \%$ reporting frequencies in each of four categories: 1-2 times per month, once per week, twice per week, and every day. After controlling for age, gender, education, and rural/urban residence, feeling "tired but happy" was found to be positively correlated with the scores on five of the MOS subscales, indicating better quality of life. This was especially true for respondents who reported high levels of physical or emotional fatigue. Open-ended comments stressed the importance of the context in which fatigue was experienced.

CONCLUSION: In addition to the dimensions of fatigue associated with a poorer quality of life, there is an aspect of fatigue associated with improved quality of life. The state of being "tired but happy" had a positive impact on quality of life and improved the level of functioning, especially in the setting of increased physical or emotional fatigue. We conclude that the context in which fatigue is experienced is important in understanding its impact on quality of life.

ASYMPTOMATIC PERIPHERAL ARTERIAL DISEASE IN A POPULATION OF WHITE, AFRICAN AMERICAN, AND HISPANIC PATIENTS. S.L. Eason ${ }^{1}$, N.J. Petersen ${ }^{1}$, M. Suarez-Almazor ${ }^{1}$, B. Davis ${ }^{2}$, T.C. Collins ${ }^{1} ;{ }^{1}$ Houston Center for Quality of Care and Utilization Studies, Houston, TX; ${ }^{2}$ University of Texas School of Public Health, Houston, TX (Tracking ID \#75118)

BACKGROUND: Asymptomatic peripheral arterial disease (PAD) has been shown to be more common than symptomatic PAD. Inadequate recognition of asymptomatic PAD may place patients at risk for increased cardiovascular morbidity and mortality. Although risk factors for asymptomatic PAD are similar to symptomatic PAD, the prevalence of asymptomatic PAD has 
not been well defined in an ethnically diverse population. The purpose of this study was to determine the prevalence of asymptomatic PAD in a population of White, African American, and English- and Spanish-speaking Hispanic patients attending a primary care clinic and to identify coexisting factors associated with a diagnosis of asymptomatic PAD.

METHODS: We screened patients 50 years of age or older attending primary care clinics of the Harris County Hospital District and the Houston VA Medical Center. Asymptomatic PAD was defined as having an ankle-brachial index (ABI) of $<0.9$ and lacking exertional leg symptoms typical of peripheral arterial disease, as defined by the San Diego Claudication Questionnaire. Logistic regression was used to compare patients with asymptomatic PAD to those without PAD, in order to identify significant associations between age, gender, race/ ethnicity, hypertension, congestive heart failure, diabetes, smoking status, alcohol consumption, activity level, and asymptomatic PAD.

RESULTS: 366 patients were screened for PAD; twenty-five, or $6.8 \%$, were diagnosed with asymptomatic PAD. Of the 25 individuals with asymptomatic $\mathrm{PAD}, 36 \%$ were White, $36 \%$ were African American, $28 \%$ were Hispanic, and $40 \%$ were female. The risk factors associated with asymptomatic PAD were diabetes mellitus ([OR] 3.8; 95\% CI 1.6, 9.0) and a history of smoking at least 1 pack of cigarettes per day ([OR $] 2.5 ; 95 \%$ CI 1.1, 6.0). As diabetes and heavy smoking were the strongest associations with asymptomatic PAD, these factors were examined to determine the presence of an interaction effect. Diabetes predicted asymptomatic PAD more sensitively than heavy smoking (64\% vs. 34\%), yet diabetes combined with heavy smoking demonstrated the highest predicted value positive (PVP, 15\%), the highest specificity $(92 \%)$, and the highest positive likelihood ratio (+LR, 2.5). Not having diabetes combined with not heavily smoking showed extremely low rates for predicting asymptomatic PAD (PVP 1\%, +LR .17).

CONCLUSION: Asymptomatic PAD is a common disease within a primary care setting. In order to diagnose and prevent further progression of PAD, the ABI should be considered as a routine screening among patients with diabetes mellitus and a history of heavy smoking attending primary care clinics. Efforts are needed to better understand the association of control of diabetes mellitus and smoking to prevent PAD, specifically asymptomatic PAD.

PREVALENCE AND CORRELATES OF MAJOR DEPRESSION AMONG A NATIONAL
SAMPLE OF ADULTS WITH CHRONIC MEDICAL CONDITIONS. L.E. Egede ${ }^{1}$, D. Zheng ${ }^{1}$; ${ }^{1}$ Medical University of South Carolina, Charleston, SC (Tracking ID \#75117)

BACKGROUND: Major depression (MD) is associated with increased healthcare utilization, costs, and poor health outcomes. Prevalence of MD is higher in patients with chronic medical conditions but precise estimates and correlates of MD in this group are poorly characterized. Data from the 1999 National Health Interview Survey (NHIS) was analyzed to estimate the prevalence and correlates of MD among patients with common chronic medical conditions. METHODS: 30,022 adults were surveyed in 1999. Eight chronic medical conditions $(\mathrm{n}=11465)$ were selected based on self-report including diabetes $(\mathrm{DM})(\mathrm{n}=1810)$, hypertension $(\mathrm{HTN})(\mathrm{n}=7363)$, cardiovascular disease $(\mathrm{CVD})(\mathrm{n}=3488)$, and chronic obstructive pulmonary disease (COPD) $(\mathrm{n}=1663)$, any cancer $(\mathrm{CA})(\mathrm{n}=2035)$, stroke (CVA) $(\mathrm{n}=$ $727)$, end-stage renal disease (ESRD) $(n=443)$, and chronic liver disease (CLD) $(n=297)$. Comorbidity was defined as having 1,2 , or $3+$ chronic conditions. MD was assessed with the Composite International Diagnostic Interview Short Form (CIDI-SF), which is a valid and reliable diagnostic interview with classification accuracy of $93 \%$ for DSM IV MD. Prevalence of $\mathrm{MD}$ across conditions, among people with $1+$ chronic conditions, and by comorbidity was determined. Multiple logistic regression was used to determine independent correlates of MD in people with $1+$ chronic conditions. Covariates included age, sex, ethnicity, education, income, employment, marital status, perceived health status, smoking status, and comorbidity. STATA was used for statistical analyses to account for the complex sampling design of the NHIS. RESULTS: Weighted prevalence of MD was as follows: DM (9.3\%), HTN (8.3\%), CVD (9.8\%), COPD (16.3\%), any CA (9.1\%), CVA (12.3\%), ESRD (17.6\%), CLD (19.0\%), and $1+$ chronic conditions $(9.0 \%)$. Weighted prevalence of MD by comorbidity was as follows: none $(4.8 \%), 1(7.8 \%), 2(10.4 \%)$, and $3+(12.5 \%)$. Controlling for covariates, whites (OR 1.3), 18 64 year olds (OR 5.2), women (OR 1.9), people with income $\$ 20,000+(\mathrm{OR} 1.3)$ the unemployed (OR 1.3), the unmarried (OR 1.9), those with perceived poor health (OR 3.7), and smokers (OR 2.0) were more likely to have MD. Odds of MD increased with increasing comorbidity (OR: $1=1.8,2=2.9,3+=3.1)$.

CONCLUSION: Prevalence of MD ranged from $8.3 \%$ to $19.0 \%$ in patients with chronic medical conditions. Odds of MD increased with increasing comorbidity. Internists should consider the diagnosis of $\mathrm{MD}$ in primary care patients with multiple chronic conditions.

THE ROLE OF PHYSICIAN SPECIALTY IN MEDICAL CARE RECEIPT: THE CASE OF PATIENTS WITH MULTIPLE SCLEROSIS. J. Elston Lafata ${ }^{1}$, L. Schultz ${ }^{1}$, C. Moon ${ }^{1}$, J. Ruether ${ }^{1}$, S.B. Elias ${ }^{1}$; ${ }^{1}$ Henry Ford Health System, Detroit, MI (Tracking ID \#76267)

BACKGROUND: While the impact of multiple sclerosis (MS) can be substantial in terms of quality of life, life expectancy is similar to age-matched controls, highlighting the appropriateness of delivering both MS-related and general preventive care to MS patients. Little is known about the receipt of either by MS patients and how such receipt differs by physician specialty.

METHODS: Using a cohort of continuously enrolled HMO patients with MS aged 18 and over receiving care in 1997 from a primary care physician (PCP), neurologist or both $(\mathrm{n}=325)$ we describe receipt of MS and general preventive care by physician specialty. MS-related care evaluated included the receipt of beta interferon, MRI testing, and referral to urology, behavioral health, occupational therapy, and physical therapy. General preventive health service receipt evaluated included pneumococcal vaccine, cholesterol screening, and, among female patients, Papanicolaou smear and mammography receipt. Study data were compiled from automated databases, medical records, and patient surveys. The relationship of service receipt to specialty of physician(s) seen (PCP only, neurologist only, vs. both as well as any PCP vs. neurologist only and any neurologist vs. PCP only) was assessed using unadjusted and adjusted generalized estimating equation approaches.
RESULTS: During the study year, over one third of patients saw only a PCP (21\%) or only a neurologist (15\%), while the remainder (64\%) sought care from both a neurologist and PCP. Patients seeing a neurologist were most likely to receive each of the $6 \mathrm{MS}$ care components assessed, however only 2 such differences were statistically significant. Those seeing any neurologist vs. PCP only were more likely to receive beta interferon (30.6 vs. $14.3 \%, \mathrm{p}=0.02)$, and those seeing a neurologist only vs. PCP only were more likely to receive ophthalmology care $(26.5$ vs. $18.4 \%, \mathrm{p}=0.02)$. After controlling for differences in patient characteristics, no statistically significant differences were found in receipt of MS care by physician specialty. Patients seeing a PCP during the year were more likely to receive each of 4 general preventive care components assessed and when differences could be tested (i.e., when zero cell counts did not exist), these remained statistically significant in adjusted models. For example, those seeing any PCP vs. neurologist only were more likely to receive lipid testing $(56.8$ vs. $20.0 \%, \mathrm{p}<0.01)$ and Pap smears (55.4 vs. $19.1 \%, \mathrm{p}<0.01)$.

CONCLUSION: Findings highlight the importance that specialists, when they serve as the principal care provider, ensure that not only needed specialty care is delivered, but also that general preventive care needs be addressed.

Funding Source: The National Multiple Sclerosis Society

SUBTHERAPEUTIC INRS ARE NOT ASSOCIATED WITH A LOWER RISK OF INTRACRANIAL HEMORRHAGE IN PATIENTS ANTICOAGULATED FOR ATRIAL FIBRILLATION. M.C. Fang ${ }^{1}$, E.M. Hylek ${ }^{1}$, J. Rosand ${ }^{1}$, S.M. Greenberg ${ }^{1}$, D.E. Singer ${ }^{1}$; ${ }^{1}$ Massachusetts General Hospital, Boston, MA (Tracking ID \#73874)

BACKGROUND: Intracranial hemorrhage (ICH) is the most feared complication associated with warfarin therapy. Despite strong evidence favoring anticoagulation for atrial fibrillation (AF), fear of ICH prompts many providers to withhold anticoagulants or target low INRs, especially in the elderly. Prior studies of ICH and anticoagulant intensity used the now outdated prothrombin time ratio, were not restricted to $\mathrm{AF}$ patients, and did not assess for concomitant aspirin use. We examined the relationship between ICH and International Normalized Ratio (INR) in patients with $\mathrm{AF}$.

METHODS: We conducted a case-control study at the Massachusetts General Hospital, searching the billing system for all patients with ICD-9 diagnoses of ICH and atrial fibrillation. Cases were included if they developed a radiographically confirmed ICH while taking warfarin. We matched each case to six control $\mathrm{AF}$ patients from our anticoagulation clinic, and reviewed medical charts for clinical information including age, INR, and aspirin use. Conditional logistic regression was used to determine the independent factors associated with ICH.

RESULTS: We identified 107 cases of warfarin-associated ICH and 642 control patients, making ours the largest ICH study to date in AF patients. Cases were older than controls (mean age 78 vs. 74 years, $\mathrm{p}<0.01$ ) and had higher INRs (mean INR 3.3 vs. $2.5, \mathrm{p}<0.01$ ). There were no differences in concomitant aspirin plus warfarin use $(20 \%$ in both cases and controls). The odds of ICH did not significantly increase until after age 85 years (referent group 70-74 years) and at INRs greater than 3.5 (referent group 2.0-2.5). Although elevated INRs were associated with higher ICH risk, most hemorrhages occurred at INRs < 3.0. Even when adjusted for age, patients with subtherapeutic INRs (less than 2.0) were as likely as those with therapeutic INRs $(2.0-3.0)$ to develop ICH (odds ratio $=1.1[0.5-2.8]$ ).

CONCLUSION: The odds of ICH did not rise significantly until after age 85 and at INRs over 3.5. Concomitant aspirin plus warfarin use was not a significant risk factor. The lower INR ranges that some guidelines have recommended for elderly patients were not associated with a lower risk of ICH. Given that the risk of ischemic stroke rises sharply at INRs $<2.0$, our study supports maintaining $\mathrm{AF}$ patients in the therapeutic anticoagulant range.

NO INCREASED RISK OF HEPATITIS C VIRUS AMONG CALIFORNIA PRISON WORKERS. R. Fox ${ }^{1}$, S. Currie ${ }^{1}$, J. Evans ${ }^{1}$, T. Wright ${ }^{1}$, A. Kindrick ${ }^{1}$, K. Page-Shafer ${ }^{1}$; ${ }^{1}$ UCSF, San Francisco, CA (Tracking ID \#76512)

BACKGROUND: Occupational exposure to hepatitis $\mathrm{C}$ virus $(\mathrm{HCV})$ is a serious concern among people who work with HCV-infected populations, such as health care workers, emergency responders and prison workers. Prior studies have found that prison inmates have a high prevalence of $\mathrm{HCV}$ infection (34\%), yet the prevalence and predictors of $\mathrm{HCV}$ infection among prison workers is not known. We investigated the prevalence and risk factors for HCV infection in a diverse sample of prison workers in California correctional institutions. METHODS: We conducted a cross-sectional study of workers in six California State correctional facilities and recruited subjects using two methods: (1) a proportional random sample of four occupational categories (guards, medical personnel, administrative, maintenance/housekeeping) using facility mailing, and (2) a volunteer sample recruited by site advertising. A total of 1,013 (819 randomly sampled and 194 volunteer sample) consented to participate in a structured interview assessing risk factors, including behavioral and occupational exposures, and $\mathrm{HCV}$ testing. Blood samples were assayed for HCV antibodies (anti-HCV) by EIA-3, and positive results confirmed by HCV-RIBA. In anti-HCV negative samples, HCV RNA was tested by a qualitative transcription mediated amplification (TMA) assay to detect recent seronegative infections. $77.9 \%$ of participants returned for results and post-test counseling.

RESULTS: The prevalence of anti-HCV was $2.1 \%$ overall. No differences were found in HCV prevalence by sampling methods (random vs. volunteer), but volunteers were older (median 43 vs $47, \mathrm{p}<0.01$ ). A high proportion $(57.3 \%$ ) of the overall sample reported ever having blood/bodily fluid exposures in the workplace (needle stick injuries, splashes with blood/body fluid, cuts with sharp objects during job duties). Bivariate analyses revealed that job category, duration of time working in prisons and occupational injuries were not associated with anti-HCV. All 21 seropositive workers had identifiable risk factors other than occupational history. In multivariate analyses controlling for age, exposures independently associated with anti-HCV were history of injection drug use (OR 88.3, 95\% CI 27.3-286.1) and history of tattooing/body piercing (OR 4.4, 95\% CI 1.2-16.4).

CONCLUSION: We found a prevalence of anti-HCV among prison workers $(2.1 \%)$ that was similar to the prevalence in the U.S. general population (1.8\%). These findings suggest that 
workers in California prisons have a very low risk of acquiring HCV via occupational exposures and are consistent with the low prevalence of HCV among health care and dental workers. Efforts towards education and infection control will help to both minimize the possible spread of $\mathrm{HCV}$ in the prison setting as well as alleviate some of the concern among workers.

RACIAL AND GENDER DIFFERENCES IN CHARACTERISTICS AND PATTERNS OF THE METABOLIC SYNDROME: A NATIONAL SURVEY. M.S. Freiberg ${ }^{1}$, L. Djousse ${ }^{1}$, H. Cabral ${ }^{2}$, R. Ellison ${ }^{1}$; ${ }^{1}$ Boston University, Boston, MA; ${ }^{2}$ Boston University School of Public Health, Department of Biostatistics, Boston, MA (Tracking ID \#76632)

BACKGROUND: Metabolic syndrome affects over $20 \%$ of the US population. The syndrome is defined as $\geq 3$ : abdominal obesity; elevated serum triglycerides or blood pressure; low high density lipoprotein (HDL) cholesterol, or high fasting serum glucose. We examined each criterion and the combination of these criteria in the metabolic syndrome to determine if gender and racial differences existed.

METHODS: We analyzed data on 8,813 adult men and women from the Third National Health and Nutrition Examination Survey (1988-1994), a cross sectional health survey of a nationally representative sample of the US population. All subjects fasted $\geq 8$ hours completed a health survey and a physical and laboratory exam. Subjects were diagnosed with the metabolic syndrome if they had $\geq 3$ : abdominal waist circumference (OBESE) $>102 \mathrm{~cm}$ in men, $88 \mathrm{~cm}$ in women; serum triglycerides (TG) $\geq 150 \mathrm{mg} / \mathrm{dL}$, serum HDL cholesterol $<40 \mathrm{mg} / \mathrm{dL}$ in men, $<50 \mathrm{mg} / \mathrm{dL}$ in women; blood pressure (HTN) $\geq 130 / 85 \mathrm{mmHg}$ or taking HTN medication; or fasting serum glucose (FBS) $\geq 110 \mathrm{mg} / \mathrm{dL}$ or taking anti-diabetic medication. We stratified the 5 criteria by gender and 3 racial groups and analyzed the 16 possible patterns. The percentages of the criteria in the patterns were calculated to determine their contribution to the diagnosis of the metabolic syndrome within each racial and gender group. Analyses were performed using SUDAAN and were weighted to reflect national estimates.

RESULTS: There were 1,227 women and 973 men in the sample who met criteria for metabolic syndrome. For all women, OBESE was the most common criterion (Mexican Americans (97.2\%), Blacks (95.8\%), and Whites (92.7\%). If OBESE was removed from the criteria, only $55 \%$ of women would have qualified for the metabolic syndrome. OBESE in men, however, was only present in $71.9 \%$ of Mexican Americans, $74.9 \%$ in Blacks, and $79.4 \%$ in Whites. For men, HTN was the most common criterion in Blacks $(84.0 \%)$ while TG was in Mexican Americans (83.5\%) and Whites (82.6\%). For women, most common pattern in Mexican Americans and Whites was OBESE, low HDL, and TG (32.7\%, 21.6\% respectively), while for Blacks it was OBESE, low HDL, and HTN (26.9\%). For men, the most common pattern in Mexican Americans was OBESE, low HDL, and TG (20\%), while for Whites it was all criteria except FBS (19.4\%). In Blacks it was OBESE, TG, and HTN (14\%).

CONCLUSION: Central obesity is the major criterion of the metabolic syndrome in women. Characteristics and patterns of the metabolic syndrome vary depending upon gender and race.

THE PREVALENCE AND BURDEN OF LOW BACK PAIN IN JAPAN: A POPULATIONBASED SURVEY. S. Fukuhara ${ }^{1}$, N. Takahashi ${ }^{1}$, S. Konno ${ }^{2}$, S. Morita ${ }^{1}$, Y. Suzukamo ${ }^{1}$, S. Kikuchi'; ${ }^{1}$ Kyoto University Graduate School of Medicine and Public Health, Kyoto, Japan; ${ }^{2}$ Fukushima Medical University, Fukushima, Japan (Tracking ID \#77171)

BACKGROUND: Low back pain (LBP) is the second most common reason for medical care visits in Japan, yet its prevalence, burden, and associated factors have never been examined in Japan. This study's objective is to estimate the prevalence of LBP in Japan, to measure its burden on patients and on society, and to identify the psychosocial factors associated with LBP-related outcomes.

METHODS: We surveyed a random sample of 4500 Japanese individuals, stratified by city size of residence and region to match the population distribution of the 2000 national census. Each of 300 sampling locations in the 43 strata contributed 15 respondents each. The selfadministered questionnaire includes items measuring LBP-related outcomes, including quality of life (QOL), missed work, and use of the health care system. Psychosocial variables were measured with the 14-item Job Content Questionnaire (JCQ), the 20-item Perceived Stress Social Questionnaire (PSS), and a 2-item job satisfaction scale. Multivariate analyses were conducted to identify associations between factors and outcomes. All analyses were adjusted for age, sex, and comorbidities.

RESULTS: The response rate was $66 \%$. The overall prevalence of LBP was estimated to be $30 \%$, and the prevalence increased with age. As measured with the SF-36, QOL for respondents with LBP was significantly lower than the population-norm, not only in the physical domains $(-0.4$ to $-1.0 \mathrm{SD}, \mathrm{P}<.01)$ but also in the mental domains $(-0.4$ to $-0.5 \mathrm{SD}$, $\mathrm{P}<.01)$. Of 845 respondents with LBP, $102(11 \%)$ reported missing work a mean of 1.2 days of work, and $281(32 \%)$ made an average of 2.4 outpatient clinic visits. Higher PSS scores and lower job satisfaction scores were significantly associated with missing work $(P=0.02,0.03)$ and visiting an outpatient clinic $(\mathrm{P}=0.03,0.04)$, independently of the severity of pain.

CONCLUSION: Low back pain has a substantial burden in Japan, both at the individual and societal levels, and is associated with psychosocial factors amenable to intervention. The high prevalence, personal implications, and demands on health care associated with LBP indicates the importance of primary care physicians in educating the public about recognizing and preventing LBP, as well as in treating it.

CARE-SEEKING BEHAVIOR FOR LOW BACK PAIN IN JAPAN: A POPULATION SURVEY. S. Fukuhara ${ }^{1}$, N. Takahashi ${ }^{1}$, S. Konno ${ }^{2}$, S. Morita ${ }^{1}$, Y. Suzukamo ${ }^{1}$, S. Kikuchi ${ }^{2}$. ${ }^{1}$ Kyoto University Graduate School of Medicine and Public Health, Kyoto, Japan; ${ }^{2}$ Fukushima Medical University, Fukushima, Japan (Tracking ID \#77192)

BACKGROUND: Low back pain (LBP) is the second most common reason for medical care visits in Japan, yet how patients seek care for LBP has never been examined. Knowing these care-seeking patterns would allow primary care physicians to intervene more effectively in this population. This study's objective is to identify how people with LBP seek medical care.
METHODS: We surveyed a random sample of 4,500 Japanese individuals stratified by city size of residence and region to match the population distribution of the 2000 national census. Each of 300 sampling locations in the 43 strata contributed 15 respondents each. The selfadministered questionnaire included items measuring LBP-related outcomes, including the use of the health care system and missed work. A clinical prediction rule verified from the previous study data distinguished people with a high probability of radiculopathy (HPR) from those with a low probability (LPR). We compared these two groups' care-seeking behaviors. Multivariate analyses were adjusted for age, sex, and comorbidities.

RESULTS: Among 865 respondents with LBP (estimated prevalence; 30\%), 28\% was classifiend into a HPR. Of these, $63 \%$ made no outpatient visits, a proportion similar to those with LPR (66\%) ( $\mathrm{P}=0.41)$. Furthermore, the HPR group sought alternative medical care as frequently as did the LPR group (chiropractic $50 \%$, massage therapy $29 \%$, acupuncture $23 \%$, Shiatsu $15 \%$ ), with or without visits to orthopedic surgeons $(56.5 \%)$ and primary care physicians (9.6\%). Significantly more HPR (16.7\%) missed their work than LPR (11.7\%), after adjustment for age, sex and comorbidity. $(\mathrm{P}=0.04)$.

CONCLUSION: People with LBP, even those with higher clinical severity, do not often seek primary care for their pain but are much more likely to seek specialty care or alternative medical care, while reporting higher work-productivity loss than those with less severity. Primary care physicians need to be more active in educating the public on prevention, assessment, and treatment of LBP.

HEPATITIS C COINFECTION IS ASSOCIATED WITH AN INCREASED SYMPTOM BURDEN IN HIV POSITIVE VETERANS. S.L. Fultz ${ }^{1}$, L. Rabeneck ${ }^{2}$, M. RodriguezBarradas ${ }^{2}$, A.C. Justice ${ }^{1} ;{ }^{1}$ Center for Health Equity Research and Promotion, VA Pittsburgh Healthcare System, Pittsburgh, PA; ${ }^{2}$ VA Medical Center and Baylor College of Medicine, Houston, TX (Tracking ID \#76236)

BACKGROUND: While infection with HIV or hepatitis C virus (HCV) is known to be associated with increased symptom burden, there is little literature examining symptom burden and its effect on quality of life (QOL) in coinfected patients.

METHODS: The Veterans Aging Cohort 3-site Study is a longitudinal study of $881 \mathrm{HIV}+$ veterans at 3 sites. The baseline survey measured QOL using Short-Form 12 (SF-12) and measured symptoms based on the ACTG symptom index which inquires about the presence of 20 symptoms over the previous four weeks, and if present how bothersome each symptom was. HCV antibody and baseline aminotransferase levels ("LFTs") were also collected, with elevated LFTs defined as levels $>1.5 \times$ upper limit of normal. Patients were categorized as (1) HCV- ( $n=400),(2) \mathrm{HCV}+$, normal LFTs ( $=87)$, and (3) HCV+, elevated LFTs $(\mathrm{n}=213)$. We used linear regression to determine the proportion of variation in QOL explained by symptoms. Nonparametric test of trend was used to determine whether symptom burden increased across the three groups.

RESULTS: Symptom burden increased across the groups (Table). For 12 of the $20(60 \%)$ symptoms bothersome increased $(\mathrm{p}<0.05)$ and trended towards increasing in one $(\mathrm{p}<=0.1)$. Symptoms explained $27 \%$ of variation in physical component score and $42 \%$ of variation in the mental component score. Viral load and CD4 count did not differ.

CONCLUSION: Hepatitis C coinfection was significantly associated with increasing symptom burden and was associated with increasing bothersome of the majority of the symptoms measured in the VACS 3 study. Patients with hepatitis C infection but normal LFTs had a larger bothersome symptom burden than hepatitis C negative patients; while hepatitis C positive patients with elevated LFTs had the largest burden. This study suggests that additional research into the management of symptoms in HIV/HCV coinfected patients has the potential of increasing patients' QOL.

Median number of symptoms by HCV and LFT status.

$\begin{array}{cccc}\begin{array}{c}\text { Hep C } \\ \text { Negative }\end{array} & \begin{array}{c}\text { Hep C } \\ \text { Positive, } \\ \text { Normal LFTs }\end{array} & \begin{array}{c}\text { Hep C } \\ \text { Positive, } \\ \text { Elevated LFTs }\end{array} & \begin{array}{c}\text { p-value } \\ \text { for test } \\ \text { of trend }\end{array} \\ 10 & 12 & 11 & <0.005\end{array}$

$\begin{array}{lrrrr}\text { Any Symptom } & 10 & 12 & 11 & <0.005 \\ \text { Bothersome Symptoms } & 7 & 8 & 9 & <0.005\end{array}$

COMPLEMENTARY ALTERNATIVE MEDICINE AND ITS USE IN PATIENTS WITH NONMALIGNANT CHRONIC PAIN. I. Genao ${ }^{1}$, A. Mechaber ${ }^{2}$; ${ }^{1}$ Society of General Internal Medicine, Avondale Estates, GA; ${ }^{2}$ University of Miami, Miami, FL (Tracking ID \#76977)

BACKGROUND: Chronic pain effects a large proportion of our patient population. Unbeknownst to their physicians, patients might be using other remedies to treat their illnesses in addition or instead of conventional therapies. The objective of our study was to determine prevalence of complementary alternative medicine (CAM) use in patients with nonmalignant chronic pain and to identify patient characteristics associated with CAM use. METHODS: The 4P (Patient-Physician Perception of Pain) study group is a multi-center collaboration formed to assess patient and physician attitudes regarding primary care management of non-malignant chronic pain using a cross-sectional study design. This arm of the study involved a 160 -item survey administered to 346 patients who suffered from nonmalignant chronic pain. Sections of the survey consisted of demographic questions, attitudes toward care, and use of conventional and CAM therapies. We analyzed the descriptive data on the use of CAM in addition to bivariate and multivariate analyses in order to identify patient characteristics associated with CAM use. In a logistic regression model, we controlled for: age, education level, pain duration, narcotic use, race, gender, depression, and patient satisfaction with health care provided.

RESULTS: Fifty-six percent (194) of patients used CAM such as cod liver oil (4\%), acupuncture $(2 \%)$, massage $(17 \%)$, garlic $(11 \%)$, primrose oil $(2 \%)$, chiropractor $(9 \%)$, meditation/yoga (7\%), traditional Chinese medicine (1\%), aromatherapy $(5 \%)$, vitamin/ mineral supplements $(37 \%)$, and others $(8 \%)$. Of the patients surveyed, sixty-eight percent 
were women, $46 \%$ were whites, $32 \%$ had an education level greater than high school. Fiftynine percent used narcotics, $37 \%$ of the patients were depressed, and $83 \%$ reported good to excellent satisfaction with the health care provided. Bivariate analysis revealed that $65 \%$ of the patients with an education level higher than high school used CAM compared to $52 \%$ of the patients with a lower education level $(\mathrm{p}=.02)$. In the logistic regression, an education level higher than high school continued to be associated with CAM use (odds ratio [OR], 1.87; 95\% confidence interval $[95 \% \mathrm{CI}], 1.13$ to 3.07 ). No other patient characteristics were statistically significant. Eighty-nine percent of patients agreed that their physicians did not ask about CAM.

CONCLUSION: More than half of patients suffering from non-malignant chronic pain use some form of CAM. Given that CAM might positively or negatively effect physicians' treatment recommendations, primary care physicians should specifically ask about CAM use as part of their chronic pain assessments. This inquiry seems particularly important for patients with higher educational attainment.

THE RELATIONSHIP BETWEEN INITIAL A1C RESULT AND RISK OF REACHING AN ACTION THRESHOLD FOR DIABETES MANAGEMENT. A. Goel ${ }^{1}$, B. Littenberg ${ }^{2}$; ${ }^{1}$ University of Vermont, Burlington, VT; ${ }^{2}$ Fletcher Allen Health Care, Burlington, VT (Tracking ID \#74418)

BACKGROUND: Diabetes is a growing problem in America. The American Diabetes Association recommends interventions for patients with an A1C greater than $8 \%$. Current surveillance protocols recommend A1C testing every 3 to 6 months. To our knowledge, no patient cohorts have been studied to determine the risk of reaching the action threshold from an initial A1C.

METHODS: We used a lab registry of all A1Cs between 1998 and 2001 processed at one academic medical center to select cases. We excluded patients under the age of 40 and patients with a history of amputation, dialysis, or kidney transplant. We only included patients with at least one additional recorded A1C values within 360 days of the initial A1C. We restricted our analysis to patients with an initial A1C greater than $6.0 \%$ and less than $8.0 \%$. We used cumulative hazard functions to determine time-to-A1C of $8.0 \%$ or greater.

RESULTS: 1872 patients met our selection criteria. We found no differences by gender or age quartiles (data not shown). We found significant differences when grouping initial A1C into $0.5 \%$ increments. At six months, $3.2 \%(95 \% \mathrm{CI}, 1.5-6.7 \%)$ of the patients with an initial A1C of $6.0-6.4 \%$ had a recorded $\mathrm{AlC}$ value of $8.0 \%$ or greater, compared to $22.8 \%(18.9-27.6 \%)$ of patients with an initial A1C of $7.5-7.9 \%$.

CONCLUSION: Changes in A1C from less than $8.0 \%$ to the $8.0 \%$ threshold seem highly dependent on the initial A1C. Future work in A1C modeling may provide insights into more efficient surveillance algorithms for patients with diabetes.

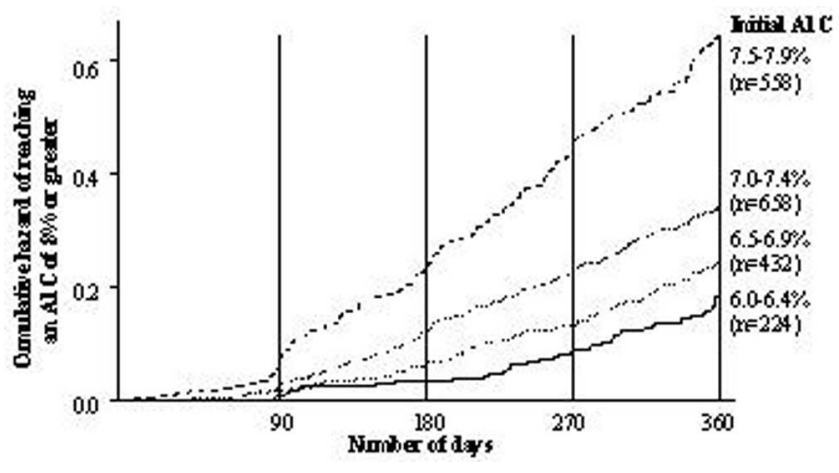

POLYPHARMACY AND MEDICATION ADHERENCE IN PATIENTS WITH TYPE 2 DIABETES. R.W. Grant ${ }^{1}$, N.G. Devita ${ }^{2}$, D.E. Singer ${ }^{1}$, J.B. Meigs ${ }^{1} ;{ }^{1}$ Division of General Medicine, Massachusetts General Hospital, Boston, MA; ${ }^{2}$ Partners Community HealthCare, Boston, MA (Tracking ID \#75114)

BACKGROUND: Evidence-based treatment of diabetes generally requires multiple medicines to control metabolic risk factors. We assessed medication adherence and predictors of suboptimal adherence in patients with diabetes and tested the hypothesis that adherence decreases with increased number of medicines prescribed.

METHODS: One-hundred and twenty-eight randomly selected patients with type 2 diabetes from a single community health center responded to a pharmacist-administered questionnaire regarding medication use. Survey data were linked to clinical data available from the electronic medical record. We assessed self-reported adherence rates for each diabetes-related medicine, barriers and attitudes regarding medication use, and last measured hemoglobin A1c, total cholesterol, and blood pressure.

RESULTS: Patients were taking a mean of $4.1( \pm 1.9)$ diabetes-related medicines. Average 7 -day adherence was $6.7 \pm 1.1$ days. Total number of medicines prescribed was not correlated with medication adherence. Adherence was significantly lower for medicines not felt to be improving current or future health (6.1 vs. 6.9 days out of $7, p<0.001$ ). Among patients on 3 or more medicines, $71 \%$ (15 of 21 patients) with sub-optimal adherence were perfectly adherent with all but one medicine. Side effects were the most commonly reported problem with medication use. Of 29 medicines causing side effects that interfered with adherence, 24 (83\%) did so for greater than 1 month and only 7 (24\%) were reported to the patient's primary care physician.

CONCLUSION: In this sample, patients reported very high medication adherence rates regardless of number of medicines prescribed. Among patients on multiple medicines, most patients with sub-optimal adherence had decreased adherence to only one medicine. Unreported side effects and a lack of confidence in immediate or future benefits were significant predictors of sub-optimal adherence.

IMPACT OF CONCURRENT MEDICATION USE ON STATIN ADHERENCE AMONG PATIENTS ENROLLED IN A HEALTH MAINTENANCE ORGANIZATION. R.W. Grant ${ }^{1}$, K.M. O'Leary ${ }^{2}$, J.B. Weilberg ${ }^{2}$, J.B. Meigs ${ }^{1}$; ${ }^{1}$ Division of General Medicine, Massachusetts General Hospital, Boston, MA; ${ }^{2}$ Department of Psychiatry, Massachusetts General Hospital, Boston, MA (Tracking ID \#75262)

BACKGROUND: Effective management of cardiovascular disease often requires long-term adherence to complex medical regimens. The impact of such polypharmacy on HMG-COA reductase inhibitor (statin) adherence is uncertain.

METHODS: We assessed the relationship between number of concurrently prescribed medicines and adherence to statins. We used pharmacy claims and health plan enrollment data for members of a single large health maintenance organization (HMO) to identify a cohort of 4413 patients from 7/99 to 6/02 who were members of the plan for at least three consecutive months before initiating statin therapy. For each patient, we determined age, gender, treatment for hypertension (HTN), ischemic heart disease (IHD) and diabetes (DM), and total number of different medicines concurrently prescribed at the time of statin initiation. We calculated two parameters of statin adherence: 1) continuous medication adherence (CMA) [number of pills dispensed/total number of days between first and last prescription], and 2) 1 year refill persistence (RP) [12 or more months of consecutive refills after initial prescription].

RESULTS: Most patients were male (61.7\%) and the mean age was $52.1 \pm 9.4$ years. Patients were prescribed a mean of $2.8 \pm 2$ total medicines (range 1-13) and were enrolled for a median of 22.7 months (IQR 13-36). Univariate predictors of higher CMA included increasing age, income, number of prescribing physicians, and number of concurrently prescribed medicines ( $p<0.0001$ for each), and treatment for HTN ( $<<0.0001)$, IHD $(\mathrm{p}=0.009)$, or DM $(\mathrm{p}=0.02)$. In a multivariate linear regression model, only age, income, HTN treatment, and number of prescribed medicines remained significant. 1-year RP was greater in men $(p=0.007)$, older patients, and patients treated for HTN or IHD ( $p<0.0001$ for each). Patients taking more medicines at baseline $(\mathrm{p}=0.03)$ and with multiple prescribing physicians $(\mathrm{p}=0.006)$ were less likely to persist for 1 year.

CONCLUSION: In this cohort of well-insured patients, increasing number of other medicines did not reduce daily adherence to statins but was associated with decreased longterm persistence. Both parameters of adherence are critical to effective management Physicians should not be deterred from prescribing statins by their patients' medical regimen complexity but do need to assess persistence with therapy.

EFFECTS OF PARTICIPATORY DECISION MAKING ON SELF-REGULATION BELIEFS, BEHAVIORS, AND OUTCOMES AMONG INNER CITY ASTHMATICS. E.A. Halm ${ }^{1}$ J. Wang ${ }^{1}$, A. Cooperman ${ }^{1}$, T. Sturm ${ }^{1}$, H. Leventhal ${ }^{2}$; ${ }^{1}$ Mount Sinai School of Medicine, New York, NY; ${ }^{2}$ Rutgers Univ., New Brunswick, NJ (Tracking ID \#75808)

BACKGROUND: Asthma morbidity and mortality is highest among inner-city populations. The aim of this study was to assess the extent to which disparities in asthma may be influenced by suboptimal self-regulation beliefs, behaviors, and participatory decision making (PDM) in a cohort of inner city adults with moderate and severe persistent asthma.

METHODS: A prospective cohort of adults admitted to an urban hospital over a consecutive 12 month period were interviewed to assess self-management knowledge, beliefs, and behaviors (medication use, peak flow $[\mathrm{PF}]$ use), and 5 items adapted from a validated PDM scale. ED visits and hospitalizations for asthma in the past year were assessed on admit and 1 month after discharge ( $86 \%$ follow-up); $28 \%$ of interviews were in Spanish.

RESULTS: Overall, 204 eligible Pts (96\%) were enrolled. Mean age was 49.8 yrs (range $19-101), 78 \%$ were female, $62 \%$ were Hispanic, $28 \%$ African-American, and $5 \%$ White. The mean asthma ED visits and hospitalizations in the $\mathrm{yr}$ prior to admission were $3.4 \pm 5.2$ and $1.4 \pm 2.6$. One month after discharge, $19 \%$ had an ED visit and $10 \%$ were readmitted for asthma. While $92 \%$ of Pts had insurance and $82 \%$ a usual place of asthma care, $66 \%$ reported ever being prescribed inhaled corticosteroids (ICS). Among these, $73 \%$ used them daily when they had symptoms (SX) and $58 \%$ took them daily when no SX $(\mathrm{p}<.05)$. While $79 \%$ had a PF meter, and $85 \%$ said it was easy to use, only $27 \%$ used it, and $71 \%$ never used PF to adjust medicines. Among the PDM items, $40 \%$ made asthma decisions together with their MD, and $49 \%$ said their MD asked them to take some responsibility for their own care; $69 \%$ said their MD never discussed how to adjust their medicines themselves, and $62 \%$ never discussed SX or PF goals, though $74 \%$ would tell their MD if a medicine was not helping. The mean PDM score was $3.2 \pm 1.1$ (range: 1 to 5 ; Cronbach's alpha $=.74$ ). Better PDM scores were associated with more desirable: chronic disease beliefs (I'll always have asthma, I have asthma even when no SX, and ICS use is important when no SX), selfregulation behaviors (ICS use when SX, ICS use when no SX, PF use, self-adjustment of medicines), and outcomes (fewer ED visits and hospitalizations in past year, and fewer ED visits and hospitalizations 1 month after discharge; $\mathrm{p}<.05$ for all Spearman correlations). CONCLUSION: Participatory decision making was associated with better self-regulation beliefs, behaviors, and outcomes. Interventions to improve inner city asthma should focus on bolstering PDM as a key, potentially modifiable factor.

ELEVATED CHOLESTEROL AND THE RISK OF INCIDENT HYPERTENSION IN MALE PHYSICIANS. R.O. Halperin ${ }^{1}$, H.D. Sesso ${ }^{2}$, J.M. Gaziano ${ }^{2} ;{ }^{1}$ Boston VA Medical CenterMAVERIC, Boston, MA; ${ }^{2}$ Brigham and Women's Hospital Division of Preventive Medicine, Boston, MA (Tracking ID \#75727)

BACKGROUND: Hypertension is a prevalent condition in the United States. There is evidence that hypertension, a major coronay risk factor, may be an early manifestation of 
atherosclerosis. This raises the possibility that high cholesterol, an established coronary disease risk factor, may be an important predictor of incident hypertension.

METHODS: Of the 22,071 men comprising the Physicians' Health Study, baseline selfreported cholesterol levels and data on other coronary risk factors were obtained from 5473 men initially free from hypertension. These men were prospectively followed for a mean of 14 years. Incident hypertension during follow-up was defined as the initiation of antihypertensive treatment, or self-reported systolic blood pressure above $140 \mathrm{mmHg}$, or diastolic blood pressure above $90 \mathrm{mmHg}$. A multivariate logistic regression model was created using cholesterol divided into quartiles as the primary exposure and incident hypertension as the outcome.

RESULTS: At baseline the mean age of the men was 53.2 years and mean self-reported cholesterol was $208.8 \mathrm{mg} / \mathrm{dl}$. A total of 1468 of the men (26.8\%) developed hypertension during follow-up. The multivariate adjusted relative risk of hypertension for men reporting a cholesterol above 240 or having been treated for hypercholesterolemia was $1.15(1.00-1.32)$. In the age adjusted model compared to men with a self-reported cholesterol of $<180 \mathrm{mg} / \mathrm{dL}$, men reporting cholesterol levels of 180 to $<205,205$ to $<237$, and $>237 \mathrm{mg} / \mathrm{dL}$ had RRs for developing hypertension of $0.93(0.78-1.11), 1.03(0.88-1.22)$, and $1.17(1.00-1.38)$, respectively ( $\mathrm{p}$, linear trend $=0.0382$ ). Multivariate models adjusted for age and other coronary risk factors modestly attenuated the age-adjusted RRs, as men reporting cholesterol levels of 180 to $<205,205$ to $<237$, and $>237 \mathrm{mg} / \mathrm{dL}$ had RRs for developing hypertension of $0.93(0.78$ $1.12), 1.04(0.88-1.23)$, and $1.12(0.95-1.33)$, respectively (p, linear trend $=0.1083)$. CONCLUSION: In this study, history of hypercholesterolemia was associated with a $15 \%$ increased risk of incident hypertension in men. Further studies are needed to better clarify the relationship between the level of cholesterol and the risk for developing hypertension, which can improve prevention strategies for hypertension.

HMG-COA REDUCTASE INHIBITORS AND OSTEOPOROSIS, A META-ANALYSIS. C. Hatzigeorgiou ${ }^{1}$, J.L. Jackson ${ }^{2}$; ${ }^{1}$ Walter Reed Army Medical Center, Washington, DC; ${ }^{2}$ Uniformed Services University of the Health Sciences, Bethesda, MD (Tracking ID \#74691)

BACKGROUND: Our specific question was: Does the use of statins reduce the rate of fractures, improve bone mineral density or bone markers of osteoporosis?

METHODS: We searched Medline, Embase, Cochrane Library, Federal Research in Progress (FEDRIP), Clinical Trials.gov, and article references. Inclusion criteria included any observational or randomized controlled trial assessing statin effects on bone health in humans. Standard meta-analytic methods were used, including sensitivity analysis, assessment of heterogeneity, and publication bias.

RESULTS: Twenty-two studies were identified: 8 evaluating statin effects in men and women, 14 exclusively women. 16 were observational studies and 6 randomized controlled trials; 3 RCTS were secondary analysis of cholesterol lowering trial data, and the remaining RCTs assessed statin effects on BMD or bone markers. Among studies including both men and women, statin use was associated with fewer hip fractures (OR: $0.61,95 \%$ CI: $0.5-0.75$ ) and improved hip BMD (SMD: 0.12, 95\% CI: 0.06-0.17), with no effect on vertebral fracture or BMD. Among studies focusing on women, hip BMD improved (SMD: 0.10, 95\% CI: $0.04-$ 0.16 ), with a nonsignificant reduction in hip fractures (OR: $0.78,95 \%$ CI: $0.52-1.16$ ), though a reduction in overall fracture rates was seen (OR: $0.82,95 \%$ CI: $0.75-0.89)$. Markers of bone turnover, alkaline phosphatase, osteocalcin, and C-telopeptide all improved (SMD: -0.28 $95 \%$ CI: -0.40 to -0.16 ; SMD: $-0.3395 \%$ CI: -0.53 to -0.14 ; SMD: $-0.17,95 \%$ CI: -0.31 to -0.1 , respectively).

CONCLUSION: HMG-CoA reductase inhibitors may have a beneficial impact on bone metabolism, bone density, and decreased fracture risk in men and women.
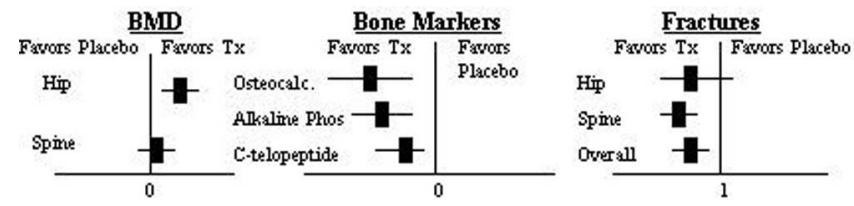

ANTIOXIDANT VITAMIN INTAKE AND SUBCLINICAL ATHEROSCLEROSIS. C. Hatzigeorgiou ${ }^{1}$, A.J. Taylor ${ }^{1}$, I. Feuerstein ${ }^{1}$, L.E. Bautista ${ }^{2}$, P.G. O'Malley ${ }^{1}$; ${ }^{1}$ Walter Reed Army Medical Center, Washington, DC; ${ }^{2}$ Uniformed Services University of the Health Sciences, Bethesda, MD (Tracking ID \#75873)

BACKGROUND: No human studies have evaluated the effect of antioxidant vitamin intake on early coronary atherogenesis. We studied the relation between vitamins and subclinical coronary artery disease to assess the possible role of antioxidant vitamins on early coronary atherogenesis in a low risk population.

METHODS: We conducted a prospective study of 730 consecutive consenting, active-duty U.S. Army personnel, 39-45 years of age, without known coronary artery disease. Each participant was assessed with the Block Dietary Questionnaire for dietary and supplemental vitamin intake. Subclinical coronary artery disease was identified by electron-beam computed tomography to quantify coronary calcification (CAC).

RESULTS: The mean age was $42( \pm 2)$ and the mean projected 10 year risk for $\mathrm{CV}$ events was $5.6 \%$. Vitamin supplements were used by $56 \%$ of the participants. The daily intake (dietary plus supplemental) of vitamin A, vitamin C, and vitamin $\mathrm{E}$ were $3270.9 \mathrm{RE}$ (retinol equivalents; $\pm 2879), 371.2 \mathrm{mg}( \pm 368.1)$, and $201.3 \mathrm{mg}( \pm 1904)$, respectively. The prevalence of $\mathrm{CAC}>0$ was
$18.7 \%$ (mean CAC score $=78.7 \pm 208$ ). There was no significant correlation between CAC score and vitamin intake: vitamin $\mathrm{A}(\mathrm{r}=0.03, \mathrm{P}=0.662)$, vitamin $\mathrm{C}(\mathrm{r}=0.02, \mathrm{P}=0.445)$, and vitamin $\mathrm{E}(\mathrm{r}=0.02, \mathrm{P}=0.990)$. In multivariate logistic regression, controlling for traditional cardiac risk factors, no association was found between the presence of coronary calcification and dietary and supplemental intake of vitamin A and C. However, the highest quartile of vitamin $\mathrm{E}$ intake was associated with a 1.77 (CI: 1.02-3.07) odds for the presence of calcification compared to the lowest quartile.

CONCLUSION: Antioxidant vitamins, whether through dietary or supplemental intake, are not significantly related to coronary calcification, implying no effect of antioxidant intake on early atherogenesis. High doses of vitamin E may confer an increased risk of calcific atherosclerosis, although this requires further study.

Daily Vitamin Intake and Coronary Calcification

$\begin{array}{cc}\text { CAC }=\mathbf{0} & \text { CAC }>\text { 0 } \\ 3184.9 & 3484.5 \\ 371.4 & 370.6 \\ 210.0 & 166.0\end{array}$

adj OR (Cl)

Vitamin A (RE)

Vitamin C (mg)

210.0

166.0

$1.00(0.99,1.01)$

$1.00(0.99,1.01)$

$1.00(0.99,1.01)$

HYPOGLYCEMIA IN STABLE INSULIN-TREATED PATIENTS WITH TYPE 2 DIABETES. R.M. Hoffman ${ }^{1}$, J.H. Shah ${ }^{2}$, W.C. Duckworth ${ }^{3}$, C.S. Wendel ${ }^{2}$, G.H. Murata ${ }^{1} ;{ }^{1}$ New Mexico VA Health Care System, Albuquerque, NM; ${ }^{2}$ Southern Arizona VA Health Care System, Tucson, AZ; ${ }^{3}$ Carl T. Hayden VA Medical Center, Phoenix, AZ (Tracking ID \#74932)

BACKGROUND: Hypoglycemia is an important complication of insulin treatment. We evaluated the incidence, severity, and predisposing factors for hypoglycemic episodes in insulin-treated patients with type 2 diabetes.

METHODS: We conducted a prospective observational study of Southwest veterans with stable, insulin-treated type 2 diabetes. Subjects were randomly selected from pharmacy databases. Electronically-recorded self-monitored blood glucose (SMBG) results were collected during 12 months of routine monitoring. We defined hypoglycemia as an SMBG reading $<=60 \mathrm{mg} / \mathrm{dL}$. Subjects graded the severity of each hypoglycemic episode $(0=$ asymptomatic, $1=$ mild/moderate symptoms, 2 = severe, with mental impairment or need for assistance. Subjects also reported any predisposing factors for each hypoglycemic episode.

RESULTS: We enrolled 344 subjects, mean (SD) age was 65.5 (9.7) years, 96.5\% were men, and $35.3 \%$ were Hispanic or African American. During a mean (SD) follow-up of $41.2(8.6)$ weeks, 176 subjects $(51.2 \%)$ documented at least one hypoglycemic reading, with a total of 1,662 episodes. Among subjects who became hypoglycemic, the median number of episodes was 4 , occurring at a rate of 0.91 episodes/4 weeks. The mean (SD) hypoglycemic blood glucose reading was $49.7(7.5) \mathrm{mg} / \mathrm{dL}$ and the mean (SD) symptom score was $0.84(0.45)$. Nearly $20 \%$ of episodes were asymptomatic, but $3.4 \%$ were severe. Subjects recorded comments for $97 \%$ of the hypoglycemic episodes. Although subjects could not identify a cause for $51.6 \%$ of the episodes, $24.1 \%$ were attributed to missing a meal, $10.8 \%$ to exercise, and only $0.7 \%$ were attributed to a medication increase.

CONCLUSION: A high proportion of stable, insulin-treated subjects developed hypoglycemic episodes, but severe hypoglycemia occurred infrequently. Hypoglycemic episodes were rarely attributed to medication increases. Efforts to achieve tight glycemic control should recognize that patient behaviors are the most common cause for hypoglycemia.

THE PREVALENCE OF METABOLIC SYNDROME IN A PUBLIC HOSPITAL. J. Huang ${ }^{1}$, H. Yu ${ }^{2}$, E. Marin ${ }^{1}$, M. Sugar ${ }^{1}$, S. Savory ${ }^{1}$, T.C. Davis ${ }^{1}$, D. Carden ${ }^{1} ;{ }^{1}$ Louisiana State University Medical Center at Shreveport, Shreveport, LA; ${ }^{2}$ Yale University, New Haven, CT (Tracking ID \#76395)

BACKGROUND: Metabolic syndrome (MS) is a constellation of metabolic disturbances including insulin resistance, glucose intolerance, elevated blood pressure (BP), dyslipidemia (DLP), and central obesity (OB). Patients with MS are at increased risk of developing diabetes mellitus $(\mathrm{DM})$ and coronary artery disease (CAD) and increased mortality from all causes including CAD. Although OB and DM disproportionately affect minorities and those served in public hospitals, the prevalence of MS in this vulnerable population has not been assessed. The purpose of this study was to determine the prevalence of MS and its major disease components in a university-based public hospital.

METHODS: A chart review was conducted on 928 patients seen in two primary care clinics between May and July of 2002. Height, weight, and waist circumference (WC) were measured with patients wearing one layer of light clothing and no shoes. Sitting blood pressure (BP) was obtained. Body mass index (BMI) was calculated as weight $(\mathrm{kg}) /$ height $\left(\mathrm{m}^{2}\right)$. Determinations of fasting blood glucose (FG), triglyceride (TG), and high-density lipoprotein cholesterol (HDL-C) were retrieved from a hospital computer database. MS was defined as $>/=$ three of the five diagnostic criteria as outlined in the National Cholesterol Education Program Adult Treatment Panel III (NCEP ATPIII). Student's t test and Chi-square test were used for the analyses of continuous and categorical variables, respectively.

RESULTS: Seventy percent of patients were black, $68 \%$ were female, and $4 \%$ were commercially insured. Mean patient age was 53 years; BMI $31 \mathrm{~kg} / \mathrm{m}^{2}$; WC $99 \mathrm{~cm}$; BP 139/ $75 \mathrm{mmHg}$; FG $120 \mathrm{mg} / \mathrm{dl}$; TG $178 \mathrm{mg} / \mathrm{dl}$; and HDL-C $48 \mathrm{mg} / \mathrm{dl}$. The prevalence of MS was $52 \%$, OB $53 \%$, HTN $70 \%$, DM $29 \%$, DLP $36 \%$, and CAD $15 \%$. Fifty-six percent of patients with MS met at least 4 diagnostic criteria. Women had a higher prevalence of MS than men $(\mathrm{P}<0.05)$ with the highest prevalence in black females $(\mathrm{P}<0.01)$. Patients with $\mathrm{MS}$ were older and had higher values of WC, FG, BP, and TG, and lower values of HDL-C $(\mathrm{P}<0.0001)$. Patients with MS were more likely to have a diagnosis of OB, HTN, DM2, DLP, or CAD $(\mathrm{P}<0.0001)$ and were more likely to have atherogenic DLP characterized by high TG and low HDL-C than patients without MS ( $\mathrm{p}<0.001)$. 
CONCLUSION: The prevalence of MS in this public hospital is higher than National data suggest. Patients with MS have significantly more co-existing OB, HTN, DM2, atherogenic DLP, and CAD than those without MS. Routine measurement of WC during clinic visits is practical and may be useful for early identification of MS.

NSAID EXPOSURE AMONG PATIENTS HOSPITALIZED FOR COMPLICATED AND NON-COMPLICATED PEPTIC ULCER DISEASE (PUD) IN ARGENTINA. J. Insúa ${ }^{1}$ P. Mavros ${ }^{2}$, E. Hunsche ${ }^{2}$, O. Tibaudin ${ }^{1} ;{ }^{1}$ Sociedad Argentina de Medicina Interna General, Buenos Aires, Argentina; ${ }^{2}$ Outcomes Research, Merck \& Co., Inc., Whitehouse Station, NJ; ${ }^{3}$ Health Services, Merck \& Co., Inc., Buenos Aires, Argentina (Tracking ID \#74676)

BACKGROUND: We examined the degree to which NSAID exposure is associated with hospitalizations for peptic ulcer disease (PUD) in Argentina.

METHODS: A retrospective case-control study was conducted by reviewing medical records of patients admitted with PUD at one of five hospitals between 1996 and 2002. Complicated PUD was defined as cases with hematemesis, melena, or GI hemorrhage. One control (admitted for reasons other than PUD) was identified for each case, matched by age, sex, and admission date. The McNemar and Wald tests were used, as appropriate, to test for differences in exposure among cases and controls.

RESULTS: 371 cases were identified. Mean age was 69 years and $46.5 \%$ were female. Based on the discharge diagnosis, $42.3 \%$ of the cases had a complicated PUD, $52.5 \%$ an uncomplicated PUD, and 5.2\% had gastritis/duodenitis. At admission, $83.6 \%$ presented with a GI bleed, and $89.0 \%$ were admitted via the emergency room. During the one year prior to admission, $58.0 \%$ of cases vs. $28.3 \%$ of controls used NSAIDs and $41.0 \%$ vs. $18.9 \%$ used aspirin (both p < 0.05 ) Conditional logistic regression analysis, adjusting for age, use of gastroprotective agents, antacids, and aspirin during the last year, number of anti-rheumatic medications, and history of asthma, hyperlipidemia, renal disease, upper GI bleeding, and musculoskeletal disease revealed that the odds of a hospitalization for PUD was 4.7 times higher among patients using NSAIDs during the prior year than non-users (OR 4.7; 95\% CI: $2.5-8.8$ ). Subgroup analyses adjusted for the same covariates showed that prior NSAID use was significantly associated with both complicated PUD (OR 4.5; 95\% CI: 1.8-11.3) and uncomplicated PUD (OR 5.8, 95\% CI $2.2-15.5)$. History of GI complications was an independent risk factor for complicated PUD (OR: 12.8) and uncomplicated PUD (OR: 31.3). Endoscopic tests were performed in $96.0 \%$ of cases vs. $0.9 \%$ of controls, and red blood cell transfusions in $56.0 \%$ vs. $6.1 \%$ (all $\mathrm{p}<0.05$ ). On average, cases were hospitalized for 5.2 days (SD: 5.8 ) and this was not significantly different from controls. $52.3 \%$ of cases were treated in the ICU, $6.2 \%$ had an upper GI surgery performed, $25.4 \%$ had a Helicobacter pylori test taken and $2.4 \%$ a urea test during the hospitalization.

CONCLUSION: Use of NSAIDs is an independent risk factor for both complicated and uncomplicated PUD patients in Argentina.

PREVALENCE OF HYPERTENSION, DYSLIPIDEMIA AND DIABETES AMONG PATIENTS IN THE VA. M.L. Johnson ${ }^{1}$, R.J. Beyth ${ }^{1}$, K. Pietz ${ }^{1}$, D. Pettitt'; ${ }^{1}$ Baylor College of Medicine, Houston, TX; ${ }^{2}$ Pfizer Global Outcomes Research, New York, NY (Tracking ID \#76129)

BACKGROUND: Hypertension (HTN), dyslipidemia (DL), and diabetes (DM) are risk factors for cardiovascular (CV) disease. The purpose of this study is to estimate the prevalence of concurrent HTN, DL and DM among patients in the VA.

METHODS: Computerized records for 69,558 patients from the Houston VA Medical Center were collected for October 1998 to September 2001. Patients were classified into mutually exclusive cohorts based upon the presence of HTN, DL or DM. We defined disease based on diagnostic, treatment, and clinical parameters using recent ATP III, ADA and JNC VI guidelines. The demographic profile and pre-existing $\mathrm{CV}$ comorbidities including myocardial infarction (MI), angina or coronary artery disease (CAD), peripheral arterial disease (PAD) and cerebrovascular disease (CEVD) were determined for each cohort.

RESULTS: The table below shows the overall age, sex, race, and CV comorbidities for patients in each cohort. 51,028 patients (73.4\%) had HTN, DL or DM. The prevalence of each condition alone was $24.3 \%, 5.1 \%$ and $1.3 \%$, respectively. 16,355 patients (23.5\%) had HTN and DL, and 9,632 patients $(13.9 \%)$ had all three conditions. The average age was 60.7 years and $96 \%$ were male. Fifty-three percent of the patients were White, 25.3\% Black, and 5.6\% Hispanic. 6.2\% had history of MI, $32.1 \% \mathrm{CAD}, 7.7 \% \mathrm{PAD}$ and $10.5 \%$ CEVD. The prevalence of preexisting $\mathrm{CV}$ comorbidities were dramatically higher for patients with concurrent conditions.

CONCLUSION: The prevalence of various combinations of concurrent HTN/DL/DM was very high in this population of predominately male, U.S. veterans. Patients with concurrent disease were more likely to have other CV comorbidities. Primary care physicians need to be aware of the mulitiple conditions for the most effective management of these patients.

Table 1. Demographic profile and comorbidities of patients with isolated and concurrent conditions

\begin{tabular}{|c|c|c|c|c|c|c|c|}
\hline & HTN alone & $\mathrm{DL}$ alone & DM alone & HTN and DL & HTN and DM & DL and DM & $\begin{array}{l}\text { HTN and DL } \\
\text { and DM }\end{array}$ \\
\hline Patients, $n$, & 16,899 & 3,534 & 930 & 16,355 & 3,032 & 666 & 9,632 \\
\hline Prevalence, $\%$ & 24.3 & 5.1 & 1.3 & 23.5 & 4.4 & 1.0 & 13.9 \\
\hline Age, mean $(s \mathrm{~g})$ & $59.0(14.5)$ & $55.9(13.1)$ & $59.9(13.6)$ & $62.8(11.7)$ & $63.3(12.7)$ & $60.5(12.0)$ & $62.9(10.7)$ \\
\hline Male, $\%$ & 95.0 & 92.7 & 94.6 & 96.9 & 96.7 & 97.1 & 97.5 \\
\hline Black, \% & 31.5 & 13.4 & 21.8 & 20.7 & 33.0 & 15.8 & 25.1 \\
\hline Hispanic, $\%$ & 5.0 & 5.1 & 8.6 & 3.9 & 9.2 & 8.8 & 7.8 \\
\hline$M I, \%$ & 2.3 & 2.6 & 0.4 & 8.7 & 4.8 & 2.4 & 11.3 \\
\hline$C A D, \%$ & 14.3 & 19.1 & 9.7 & 45.3 & 26.7 & 22.2 & 49.9 \\
\hline PAD, $\%$ & 4.0 & 2.7 & 3.3 & 9.6 & 9.4 & 2.4 & 13.0 \\
\hline CEVD $\%$ & 6.9 & 3.6 & 3.1 & 12.9 & 14.3 & 4.2 & 15.0 \\
\hline
\end{tabular}

TREATMENT PATTERNS AND GOAL ATTAINMENT FOR PATIENTS WITH HYPERTENSION, DYSLIPIDEMIA AND DIABETES IN THE VA. M.L. Johnson ${ }^{1}$ R.J. Beyth ${ }^{1}$, K.C. Pietz ${ }^{1}$, D. Pettitt ${ }^{2}$; ${ }^{1}$ Baylor College of Medicine, Houston, TX; ${ }^{2}$ Pfizer Global Outcomes Research, New York, NY (Tracking ID \#76615)

BACKGROUND: The purpose of this study is to describe treatment patterns and attainment of treatment goals for hypertension (HTN), dyslipidemia (DL), and diabetes (DM) among patients in the VA health care system.METHODS: Computerized patient records for 69,558 patients from the Houston VA Medical Center were collected for the period October 1998 to September 2001. Patients were identified using diagnostic, treatment and clinical parameters and were classified into inception cohorts of newly diagnosed and treated patients with isolated or concurrent HTN, DL or DM. The frequency of utilizing distinct classes of medication therapy was determined. All patients were tracked in the database for at least one year. Goal attainment based on JNC-VI, NCEP, and ADA guidelines was determined at the end of the study period.

RESULTS: There were a total of 9,509 patients identified in the inception cohorts. Over hal of these ( $n=5,482$ or $57.2 \%$ ) had isolated HTN. Rates of no treatment ranged from $31.6 \%$ to $62 \%$ for patients with isolated conditions. Rates of multiple drug class use ranged from $1.5 \%$ to $20.2 \%$ in patients with isolated conditions, but from $2.2 \%$ to $54.9 \%$ in patients with more than one condition. Average length of followup was 1.75 years. Goal attainment in patients with HTN alone was about $45 \%$, but decreased to $27 \%$ in patients with HTN, DL, and DM. For patients with DL or DM, goal attainment was highest if they had all three conditions, rather than in isolation.

CONCLUSION: Based upon the utilization of drugs and multiple therapeutic classes, physicians appear to be treating patients with multiple conditions more aggressively, which may reflect these patient's increased risk for coronary heart disease. However, less than $50 \%$ of patients reached therapeutic goals for any condition. Further research is needed to examine determinants of patient adherence and physician practice patterns to improve outcomes.

Table 1. Drug class use and goal attainment for patients with isolated and concurrent conditions

\begin{tabular}{|c|c|c|c|c|c|c|c|}
\hline & HTN alone & DL alone & DM alone & HTN and DL & HTN and DM & $\mathrm{DL}$ and $\mathrm{DM}$ & $\begin{array}{c}\mathrm{HTN} \text { and } \mathrm{DL} \\
\text { and DM }\end{array}$ \\
\hline Patients, $n(\%)$ & $5,482(57.6)$ & $1,225(12.9)$ & $253(2.7)$ & $1,776(18.7)$ & $332(3.5)$ & $85(0.8)$ & $356(3.7)$ \\
\hline \multicolumn{8}{|l|}{ HTN } \\
\hline No drugs, $\%$ & 53.2 & & & 40.8 & 32.5 & & 17.1 \\
\hline 1 class, $\%$ & 26.6 & & & 29.7 & 27.7 & & 27.0 \\
\hline $2+$ classes, $\%$ & 20.2 & & & 29.5 & 39.8 & & 54.9 \\
\hline At goal, $\%$ & 45.2 & & & 42.5 & 39.8 & & 27.0 \\
\hline \multicolumn{8}{|l|}{$\mathrm{DL}$} \\
\hline No drugs, $\%$ & & 62.0 & & 54.4 & & 47.1 & 30.1 \\
\hline 1 class, $\%$ & & 36.5 & & 43.4 & & 47.1 & 66.6 \\
\hline $2+$ classes, $\%$ & & 1.5 & & 2.2 & & 5.8 & 3.4 \\
\hline At goal, $\%$ & & 37.8 & & 36.2 & & 23.2 & 40.9 \\
\hline \multicolumn{8}{|l|}{ DM } \\
\hline No drugs, $\%$ & & & 31.6 & & 34.6 & 34.1 & 20.8 \\
\hline 1 class, $\%$ & & & 63.6 & & 58.4 & 57.6 & 64.9 \\
\hline $2+$ classes $\%$ & & & 4.7 & & 6.9 & 8.3 & 14.3 \\
\hline At goal, $\%$ & & & 31.6 & & 37.0 & 29.8 & 49.3 \\
\hline
\end{tabular}

ATRIAL FIBRILLATION IS ASSOCIATED WITH ELEVATED C-REACTIVE PROTEIN IN PATIENTS WITH CORONARY ARTERY DISEASE. A.S. Kanderian ${ }^{1}$, N. Seshadri ${ }^{1}$, D.O. Martin ${ }^{1}$, D.R. Van Wagoner ${ }^{1}$, D.P. Chew ${ }^{1}$, D. Bhatt ${ }^{1}$, M.K. Chung ${ }^{1} ;{ }^{1}{ }^{1}$ Cleveland Clinic Foundation, Cleveland, $\mathrm{OH}$ (Tracking ID \#76894)

BACKGROUND: Elevated C-reactive protein (CRP), a marker of systemic inflammation, has been associated with prediction of future coronary disease (CAD), stroke and recently with persistence of atrial fibrillation (AF). We tested the hypothesis that in patients who have manifest CAD, higher inflammatory burden, as marked by elevated CRP, is associated with AF. METHODS: Patients undergoing percutaneous coronary intervention (PCI) had baseline ECGs and CRP levels assayed. Patients in AF prior to PCI were compared to those in sinus rhythm. Data were analyzed using t- or Wilcoxon rank-sum tests.

RESULTS: Of 1335 patients, 38 (2.8\%) were in AF on baseline ECG. Unstable angina (UA) or acute myocardial infarction within 30 days (AMI) was present in $23(60.5 \%) \mathrm{AF}$ pts and in $820(63.4 \%)$ non-AF pts, $\mathrm{p}=\mathrm{ns}$. AF patients had significantly higher $\mathrm{CRP}$ levels than non- $\mathrm{AF}$ patients $(3.04 \pm 4.86$ vs $1.52 \pm 2.96 \mathrm{mg} / \mathrm{dl}, \mathrm{p}=0.002)$. Other univariate predictors of higher CRP included UA $(1.67 \pm 3.04$ vs $1.17 \pm 2.58 \mathrm{mg} / \mathrm{dl}, \mathrm{p}<0.001)$, AMI $(3.59 \pm 5.02$ vs $1.7 \pm 2.05 \mathrm{mg} /$ $\mathrm{dl}, \mathrm{p}<0.001)$ and decreased LVEF $(\mathrm{r}=-0.190, \mathrm{p}<0.001)$. Multivariate analysis showed that AF, AMI, LVEF, COPD, and renal failure were independent predictors of higher CRP (Table). CONCLUSION: In patients with CAD undergoing PCI, AF was associated with higher CRP. This association remained significant after controlling for other comorbidities. These data further support a potential role for inflammation in the pathophysiology of $\mathrm{AF}$.

\section{Adjusted CRP, mg/dI}

Atrial Fibrillation

MI within 30 days

LVEF $<40 \%$

COPD

Renal Failure

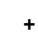

3.04

3.71

2.48

2.28

2.51
1.07

1.43

1.51
1.50 $p$ value

0.009

$<0.001$

$<0.001$

0.022
$<0.001$ 
DIABETIC CONTROL AMONG VETERANS WITH SEVERE MENTAL ILLNESS B.P. Kinosian ${ }^{1}$, J. Streim ${ }^{1}$, S. Sayers ${ }^{1}$, M. Cary ${ }^{1}$, T. Tenhave ${ }^{1}$, I.R. Katz ${ }^{1} ;{ }^{1}$ University of Pennsylvania, Philadelphia, PA (Tracking ID \#77007)

BACKGROUND: Diabetes is a prevalent chronic illness which may be more prevalent among patients with schizophrenia and those treated with atypical antipsychotic agents. Current care guidelines make no special modifications for the impact of severe mental illness (SMI) on recomended management. It is not known if recognition and care for diabetics is worsened by SMI (schizophrenia, bipolar disorder, post-traumatic stress disorder [PTSD] ). HYPOTHESES: (1) Poor control (HbA1c $>8$ ) is more prevalent among diabetic veterans with SMI and (2) good control $(\mathrm{HbA} 1 \mathrm{c}<6)$ is less prevalent among diabetic veterans with SMI, compared to diabetic veterans without SMI.

METHODS: Cohort of all ICD9-CM-identified diabetic veterans receiving outpatient care at Philadelphia VAMC, assembled for 2000-01. Glycemic control (HbA1c values), lipid, eye and foot examinations (categorical) from 1999-2001 were abstracted from the electronic record, with a $0-4$ adherence score contructed. Mixed effects models were separately developed to predict good and poor control.

RESULTS: Among 6,084 diabetic veterans, 4,496 (74\%) had 1 or more HbAlc tests $(12,463$ tests, 95 th centile 5 tests, median 2 tests). SMI patients had more tests $(3.17$ vs. $2.7, \mathrm{p}<.0001)$, with greater variability in values $(\mathrm{CV}=.14$ vs. 0.11$)$. SMI was associated with poorer control (OR 1.3, p = .03) with non-significant trends for schizophrenia (OR 1.4, p = .10), and bipolar disorder (OR 1.7, $\mathrm{p}=.08)$ and a significant trend for improvement over the year (OR .48, $\mathrm{p}<.0001)$. (See TABLE) Good control was unassociated with SMI. Guideline adherence score was associated with poorer control (OR 1.25, $\mathrm{p}<.0001)$.

CONCLUSION: Diabetic veterans with comorbid SMI (schizophrenia and bipolar disorder, but not PTSD) are more likely to have poor glycemic control, and may require modifications in diabetic management strategies to account for their psychiatric comorbidity. Poor control may be a signal to providers for greater adherence to care guidelines.

$\begin{array}{lcccc} & \text { No SMI } & \text { Schizo } & \text { Bipolar } & \text { PTSD } \\ \mathrm{n} & 3827 & 229 & 91 & 349 \\ \text { last HbA1c } & 7.27 & 7.46 & 7.59 & 7.28 \\ \text { Mean HbA1c } & 7.50 & 7.74 & 7.71 & 7.57\end{array}$

FACTORS THAT INFLUENCE CHRONIC PAIN PATIENTS' SATISFACTION WITH PRIMARY CARE OFFICE VISITS. J.E. Kurz ${ }^{1}$, I.A. Chen ${ }^{2}$; ${ }^{1}$ University of North Carolina School of Medicine, Chapel Hill, NC; ${ }^{2}$ Eastern Virginia Medical School, Norfolk, VA (Tracking ID \#75510)

BACKGROUND: Chronic pain is often under-treated by clinicians in the primary care setting. One measure of successful pain management is patient satisfaction. Our study examines factors that influence a chronic pain patient's degree of satisfaction with their primary care provider.

METHODS: The 4P study (Patient and Physician Perception of Pain) is a multi-center, cross sectional study of patients' and their physicians' attitudes and preferences regarding chronic pain care. A survey was administered to patients with 3 months or more of chronic, non-cancer pain just prior to their doctor's appointment, and a follow-up phone interview was conducted 2 weeks later. Our main outcome was patient satisfaction at the 2 -week follow-up. We performed bivariate analysis examining factors associated with patient satisfaction and then combined these factors in a logistic regression model controlling for potentially confounding variables such as race, gender, severity of pain, depression, narcotic use, continuity of care, and discordance between patient and physician in rating the patient's level of pain severity.

RESULTS: We report preliminary data based on the first 346 patients surveyed: $70 \%$ were female, $76 \%$ were insured, and $33 \%$ said they were depressed. Discordance between patient and physician of 2 or more points on the 10 -point pain scale occurred in $46 \%$ of office visits. Of 234 completing the 2-week follow-up so far, $153(66 \%)$ indicated they were satisfied with their care. Of those patients who said their MD educated them about their pain, $73 \%$ reported they were satisfied with their care compared to only $25 \%$ satisfaction in those who reported not being educated $(\mathrm{p}=.001)$. Age, gender, race, pain severity, discordance and depression were not associated with patient satisfaction. After combining education with these variables in the logistic regression model, we found that educating the patient about their pain remained the only significant factor associated with patient satisfaction, OR $3.2(95 \%$ CI 1.4, 7.0).

CONCLUSION: Patient education about chronic pain is associated with better patient satisfaction in the primary care office setting. Surprisingly, patient satisfaction is not associated with discordance or pain severity. Further study should investigate what specific educational content leads to improved patient satisfaction and whether satisfaction results in better outcomes.

SYMPTOM DISTRESS AND QUALITY OF LIFE AT THE END OF LIFE: A COMPARISON OF PATIENT, CAREGIVER AND NURSE ASSESSMENTS. J.S. Kutner ${ }^{1}$, D. Fairclough ${ }^{1}$, B. Beaty ${ }^{1}$, C.T. Kassner ${ }^{1}$, J.F. Steiner ${ }^{1}$; ${ }^{1}$ University of Colorado Health Sciences Center, Denver, CO (Tracking ID \#74176)

BACKGROUND: We described and compared symptom distress and quality of life among persons receiving hospice/palliative care from the perspective of patients and proxies (caregivers and nurses). We hypothesized that 1) proxy respondents would rate symptom (sx) distress higher and quality of life (QOL) lower than would patients, 2) patient and nurse assessments would be highly correlated, and 3) correlations would be higher for physical than for psychological issues.

METHODS: A prospective cohort study in the Population-based Palliative Care Research Network (PoPCRN), provided data [Condensed Memorial Symptom Assessment Scale (MSAS, range $0-4$, higher $=$ more sx distress), McGill Quality of Life Questionnaire (MQOL, range $0-10,0=$ bad, $10=$ good)] at hospice admission, 1 and 2 weeks, monthly, and at death or discharge. English-speaking adult patients $(\mathrm{P})$ receiving care from PoPCRN sites (if able), their caregivers $(\mathrm{C})$ (if available), and nurses $(\mathrm{N})$ participated. We used a multivariate mixed effects model to assess the relationships between the 3 groups.

RESULTS: 86 patients from 10 hospices participated: $53 \%$ female, $60 \%$ with a cancer diagnosis, 95\% non-Hispanic white, mean age 72 (range 33-100). Respondents differed on only 3 of the 10 possible sx and QOL scales/subscales (see table): 1) Nurses rated supportrelated QOL lower than caregivers or patients, 2) Caregivers rated physical well-being-related QOL lower than nurses or patients, and 3) Nurses rated physical sx distress lower than caregivers or patients. No differences were found for any of the other MQOL (overall, total, existential well-being, psychological, physical sx) or MSAS (global distress index, psychological) scores. Overall, nurse \& patient scores appeared to be more highly correlated than were the caregiver \& patient. Correlations tended to be lower for physical symptom-related QOL and higher for overall QOL (see table).

CONCLUSION: Respondent types differed little in their ratings of sx or QOL. Where differences did exist, proxies rated both QOL and sx distress lower than did patients. The assessments of all respondents correlated only moderately across all QOL and sx distress measures. Clinicians should seek the perspectives of all involved parties when seeking to decrease sx distress and maximize QOL at the end of life. From a research perspective, these findings indicate the need to incorporate data from all available respondents when one encounters missing sx or QOL data.

\begin{tabular}{|c|c|c|c|c|c|}
\hline & MQOL Support & $\begin{array}{l}\text { MQOL Physical } \\
\text { well-being }\end{array}$ & $\begin{array}{c}\text { MSAS } \\
\text { Physical sx }\end{array}$ & $\begin{array}{l}\text { Lowest } \\
\text { Correlations } \\
\text { (scale) }\end{array}$ & $\begin{array}{l}\text { Highest } \\
\text { Correlations } \\
\text { (scale) }\end{array}$ \\
\hline \multicolumn{6}{|l|}{ pondent pair } \\
\hline & $\begin{array}{c}7.62 \text { vs. } 8.55 \\
(p=0.01)\end{array}$ & $\begin{array}{c}4.53 \text { vs. } 4.54 \\
(p=0.97)\end{array}$ & $\begin{array}{c}1.45 \text { vs. } 1.71 \\
(\mathrm{p}=0.03)\end{array}$ & $\begin{array}{c}0.28 \\
\text { (MQOL Physical sx) }\end{array}$ & $\begin{array}{c}0.61 \\
\text { (MQOL Global) }\end{array}$ \\
\hline & $\begin{array}{l}8.47 \text { vs. } 8.55 \\
(p=0.86)\end{array}$ & $\begin{array}{l}2.94 \text { vs. } 4.54 \\
(p<0.01)\end{array}$ & $\begin{array}{c}1.75 \text { vs. } 1.71 \\
(\mathrm{p}=0.79)\end{array}$ & $\begin{array}{c}0.21 \\
\text { (MQOL Support) }\end{array}$ & $\begin{array}{c}0.54 \\
\text { (MQOL Global) }\end{array}$ \\
\hline & $\begin{array}{c}7.62 \text { vs. } 8.47 \\
(p=0.02)\end{array}$ & $\begin{array}{c}4.53 \text { vs. } 2.94 \\
(\mathrm{p}<0.01)\end{array}$ & $\begin{array}{c}1.45 \text { vs. } 1.75 \\
(\mathrm{p}=0.03)\end{array}$ & $\begin{array}{c}0.14 \\
\text { (MQOL Physical sx) }\end{array}$ & $\begin{array}{c}0.64 \\
\text { (MQOL Total) }\end{array}$ \\
\hline
\end{tabular}

A RANDOMIZED TRIAL OF TELEPHONE DISEASE STATE MANAGEMENT FOR CONGESTIVE HEART FAILURE. W.C. Dunagan ${ }^{1}$, B. Littenberg ${ }^{2}$, G.A. Ewald ${ }^{1}$, C.A. Jones ${ }^{1}$, V.B. Emery ${ }^{1}$, B.M. Waterman ${ }^{1}$, J.G. Rogers ${ }^{1}$; ${ }^{1}$ Washington University, St. Louis, MO; ${ }^{2}$ University of Vermont, Burlington, VT (Tracking ID \#75878)

BACKGROUND: Readmission for congestive heart failure (CHF) after hospital discharge represents a significant burden to the patient and the health system.

METHODS: 151 adults hospitalized for CHF were enrolled in a prospective, randomized, controlled study of a nurse-administered, telephone-based disease management program. Outcomes included hospital readmission, mortality, functional status, and satisfaction with care. The intervention included scheduled telephone calls by specially trained nurses. The nurses promoted patient self-management, provided dietary counseling, and encouraged adherence to guideline-based therapy prescribed by primary physicians. The nurses also screened for increased heart failure symptoms. When there was evidence of exacerbation, supplemental ("rescue") diuretics were administered, or the primary physician was contacted for instructions. Patients were called within 3 days of discharge and then at least weekly for 2 weeks. Call frequency then varied based on the patient's clinical status and self-management abilities. Control subjects received no post-enrollment contact.

RESULTS: The mean age was 70 years. $56 \%$ of the subjects were female. $57 \%$ were AfricanAmerican. $80 \%$ were NYHA class III or IV. $87 \%$ had at least one additional serious co-morbid illness. $91 \%$ were retired, disabled, or unemployed. The control and intervention groups were well matched with regard to demographics, left ventricular function, NYHA classification, and comorbidity. Over 1 year, intervention patients had a longer time to encounter (hospital or emergency room visit) than control patients ( $\mathrm{HR}=0.67 ; 95 \% \mathrm{CI} 0.47-0.96 ; \mathrm{P}=.029)$. They also had longer time to hospital readmission ( $\mathrm{HR}=0.67$; CI 0.46-0.99; $\mathrm{P}=.045)$ and $\mathrm{CHF}-$ specific readmission $(\mathrm{HR}=0.62$; CI $0.38-1.02 ; \mathrm{P}=.062)$. Cox proportional hazard analysis controlling for ejection fraction, NYHA class, and comorbidity yielded little change. For example, for time to encounter, $\mathrm{HR}=0.60$ (CI $0.35-1.02, \mathrm{P}=0.062$ ). Intervention and control patients did not differ with respect to survival, functioning, or satisfaction with care.

CONCLUSION: A nurse-administered, telephone-based disease management program including frequent monitoring, judicious use of supplemental diuretics, and patient education significantly delayed subsequent healthcare encounters. The program had minimal impact on functional status, satisfaction, or mortality.

DIABETES DOCUMENTATION DURING PREGNANCY: A COMPARISON OF THREE SOURCES. A. Maguire ${ }^{1}, \mathrm{H}$. Ashley ${ }^{2}, \mathrm{~K}$. Frommell ${ }^{3}, \mathrm{R}$. Kirby ${ }^{4} ;{ }^{1}$ University of Wisconsin Medical School, Department of Medicine, Milwaukee, Wl; ${ }^{2}$ University of Minnesota Medical School, Department of Obstetrics and Gynecology, Minneapolis, MN; ${ }^{3}$ University of Wisconsin Medical School, Madison, WI; ${ }^{4}$ University of Alabama School of Public Health, Birmingham, AL (Tracking ID \#74808)

BACKGROUND: Vital statistics are an important source of epidemiologic data, however under-reporting of maternal chronic conditions on birth certificates has been reported in several states. We examined the documentation of maternal diabetes, a risk factor for perinatal morbidity, at an urban Wisconsin medical center with one of the largest delivery volumes in the state.

METHODS: Objectives:1.To assess the accuracy of maternal diabetes documentation on Birth Certificates. 2. To compare the prevalence of Gestational Diabetes Mellitus (GDM) and Preexisting Diabetes Mellitus (DM) in three administrative data sources. The study population includes all 13,624 deliveries at Aurora Sinai Medical Center (ASMC) in Milwaukee from 
1996-1999. A retrospective review of delivery records including some prenatal records was performed. Diabetes status was determined using the treating clinician's impression and OGTT. Chart review data was compared with three sources: Birth Certificate, Hospital Discharge Database, and the ASMC Perinatal Database. Analysis included chi-square and Student's t-tests.

RESULTS: 465 women (3.4\% of the population) were identified as having either GDM or DM by at one or more sources. $69 \%$ were African American. $17.7 \%$ were $>35$ yrs of age. $12.2 \%$ had no documented GDM or DM in the available records. 401 women had GDM or DM confirmed by chart review. 313 (68.5\%) had GDM and 88 (18.9\%) had DM. Hospital data identified $84.5 \%$ of the combined group of GDM and DM. Birth Certificate data was more sensitive, identifying $91 \%$ of diabetic women. The Perinatal Database overestimated GDM. Birth Certificates correctly identified $264(84.3 \%)$ of GDM and $56(63.6 \%)$ of DM with an overall accuracy of $79.9 \%$. 11 women $(19.6 \%)$ were reported to be diabetic on the birth certificate despite a lack of documentation in the delivery records.

CONCLUSION: Our analyses indicate that these sources provide varying estimates of GDM and DM in this population. Birth certificates underestimate the prevalence of both GDM and DM, but are least accurate for documenting DM, likely due to misclassification of pre-existing DM as GDM. Discrepancies in Birth Certificate data may reflect lack of clarity regarding diabetes type and lead to inadequate post-partum management.

EFFECT OF PREDNISONE THERAPY ON BLOOD PRESSURE IN PATIENTS TREATED FOR GCA. B.F. Mandell ${ }^{1}$, T. Lenardo ${ }^{1}$, G. Locker ${ }^{1}$, J. Qureshi ${ }^{1}$, G.S. Hoffman ${ }^{1} ;{ }^{1}$ Cleveland Clinic Foundation, Cleveland, OH (Tracking ID \#77133)

BACKGROUND: Prednisone (pred) is the primary therapy for giant cell arteritis (GCA). Though prednisone has been implied to cause HTN, this has not been convincingly demonstrated in clinical studies.

METHODS: We analyzed data from a prospective, placebo- controlled trial of pred with or without methotrexate (MTX) in GCA for pred induced HTN. All GCA patients (creat <2.0) enrolled in this trial received pred $1 \mathrm{mg} / \mathrm{kg} /$ day. $\mathrm{BP}$ was to be measured at all visits. The primary end point of our retrospective analysis of the data was BP change from the baseline, measured at 2 weeks following initiation of pred.

RESULTS: Data from 44/89 of the patients enrolled in the initial study were evaluable in this analysis of HTN following pred. therapy. Patients who had manipulation of anti-hypertensive medications because of BP elevation (independent of steroid effect) between 0 and 2 weeks ( $n=$ $2)$ and those in whom pred was started prior to initial visit form completion $(n=39)$, or were missing BP data at 2 and 4 weeks $(n=4)$ were excluded from this analysis. Pts. were evaluable if baseline and either 2 or 4 week BP were recorded. BP at the scheduled 2 and 4-week visit following start of pred. therapy was compared to the pre-pred BP; difference was evaluated for each patient. There was $99 \%$ power to detect a difference of $10 \mathrm{~mm}$ with a sample size of 33.There was no statistically significant difference between the group mean syst or diast $\mathrm{BP}$ at 2 ( $\mathrm{p}$ values of 0.96 and $0.63, \mathrm{n}=42$ ) or at 4 weeks ( $\mathrm{p}$ values 0.58 and $0.82, \mathrm{n}=33$ ) when compared to baseline. But the \# of patients with $>5 \mathrm{~mm} \mathrm{Hg}$ increase in SBP at 2 weeks was 19 $(44 \%)$ and at 4 weeks was $14(42 \%)$; in DBP was $18(42 \%)$ at 2 weeks and $11(33 \%)$ at 4 weeks. \# Pts who had $>10 \mathrm{~mm} \mathrm{Hg}$ SBP increase at 2 weeks was 12 (28\%) and at 4 weeks was 7 (21\%); 10 $(23 \%)$ had $>10 \mathrm{~mm}$ inc DBP at 2 weeks and $9(27 \%)$ at 4 weeks. SBP and DBP increase at 2 weeks was more common in patients who had baseline HTN, at 4 weeks this relationship was only seen with DBP.

CONCLUSION: Although pred therapy did not cause a change in the group mean diastolic or systolic BP, it did cause $>10 \mathrm{~mm}$ elevation in systolic or diastolic BP in $\sim 25 \%$ of patients with GCA, median age 74 (55-89). It is therefore important to closely monitor BP in older patients who are started on high dose prednisone at 2 weeks after start of therapy, especially in patients who have a history of baseline hypertension.

METABOLIC SYNDROME-DEFINING FACTORS AND PROSTATE CANCER MORTALITY. S. Marcella ${ }^{1}$, V. Dombrovskiy ${ }^{2}$; ${ }^{1}$ UMDNJ-Robert Wood Jobnson Medical School, Whitehouse Station, NJ; ${ }^{2}$ UMDNJ-Robert Wood Johnson Medical School, New Brunswick, NJ (Tracking ID \#76329)

BACKGROUND: Most prostate cancer (CaP) occurs in older men. These patients often have various chronic illnesses before cancer detection. It is possible that such comorbidity may actually modify the biology and outcome of $\mathrm{CaP}$. Westernization is associated with an increased incidence of clinically significant $\mathrm{CaP}$ as well as Syndrome " $\mathrm{X}$ " (SX) or the "metabolic syndrome". SX is defined by central obesity, insulin resistance, hypertension, dyslipidemia, atherosclerosis, and hyperuricemia. One study has shown an association between SX and colorectal cancer mortality. We speculate that the presence of this syndrome may be associated with more lethal $\mathrm{CaP}$. Specifically, we hypothesize that men who die of metastatic $\mathrm{CaP}$, in comparison with men of the same age but without evidence of symptomatic $\mathrm{CaP}$, are more likely to have SX-defining factors.

METHODS: This is a case-control study with frequency matching for age, race, and the time available for diagnosing SX factors. Cases are men (ages 55-70) who died from metastatic CaP from 1997 to 2000. They were sampled from a population-based cancer registry. Controls were sampled from the same population from which cases arose. Time available to have SX factors diagnosed was nearly identical for both groups. Only factors diagnosed before the case diagnosis of $\mathrm{CaP}$ were counted to reduce observation bias. The number of factors present in each subject were used to create an index $(0-6)$. The chi-square test for categorical variables and Student's t-test for continuous variables were used in bivariate analysis. Multivariable logistic regression was used for modeling the outcome as a function of the number of metabolic risk factors present controlling for potential confounders (age, socioeconomic status, and race). RESULTS: Two thirds of cases and one half of controls had one or more SX factors diagnosed. The following SX factors were significantly associated with $\mathrm{CaP}$ mortality: hypertension $(\mathrm{OR}=2.295 \% \mathrm{CI} 1.46,3.4)$, atherosclerosis $(\mathrm{OR}=1.895 \% \mathrm{CI} 1.1,2.8)$, insulin resistance $(\mathrm{OR}=2.195 \% 1.1,4.1)$, and abdominal obesity $(\mathrm{OR}=1.795 \% \mathrm{CI} 1.05,2.7)$. In multivariable logistic regression analysis with adjustment for age, race, and education, hypertension and atherosclerosis retained their significance with ORs of 1.9 and 1.8 respectively. The model including the index variable for the number of component SX factors revealed a graded response with ORs ranging from 2.0 for one factor, 2.8 for 2-3 factors, and 3.4 for four factors (no SX factors as referent). All ORs for index categories were statistically significant with a lower $95 \%$ CI greater than 1.0 .

CONCLUSION: Metabolic syndrome-defining conditions appear to be risk factors for $\mathrm{CaP}$ mortality, and the level of this risk is graded in direct proportion to the number of component factors present in an individual.

THE EVOLVING PATTERN OF STATIN USE AMONG PATIENTS IN A MANAGED CARE POPULATION. B.D. McCarthy ${ }^{1}$, M. Ulcickas Yood ${ }^{2}, K$. Wells ${ }^{1}$, G. Kucera ${ }^{1}$, J. Kempf ${ }^{3}$, S. Oliveria ${ }^{2}$, P. Stang ${ }^{2} ;{ }^{1}$ Henry Ford Health System, Detroit, MI; ${ }^{2}$ Galt Associates, Sterling, VA; ${ }^{3}$ AstraZeneca, Wayne, PA (Tracking ID \#77063)

BACKGROUND: Studies indicate that a substantial proportion of patients who take statins fail to meet cholesterol treatment targets. Our objective was to examine changes in prescribing patterns and LDL control over time.

METHODS: Using computerized billing, prescription, and laboratory data, we studied 16,728 HMO members >= 18 years of age receiving care from Henry Ford Medical Group physicians and continuously enrolled for at least one year, who filled their first prescription for a statin between 1/1/97 and 6/30/01. LDL targets, based on ATP guidelines, were assigned to each patient according to risk factors identified through laboratory and billing data. RESULTS: Mean LDL at therapy initiation decreased from $172 \mathrm{mg} / \mathrm{dl}$ in 1997 to $159 \mathrm{mg} / \mathrm{dl}$ in 2001 ( $\mathrm{p}<0.01)$. The proportion of patients prescribed starting dose strengths above the usually recommended level differed for individual statins $(\mathrm{p}<0.001)$; but overall, this proportion increased from $5 \%$ in 1997 to $9 \%$ in 2001 ( $p$ trend $<0.001$ ). Follow-up LDL testing occurred more promptly and consistently during the later years. The combined endpoint of not reaching LDL goal or not receiving LDL testing within 1 year decreased from $46 \%$ in 1997 to $31 \%$ in $2001(\mathrm{p}<0.001)$.

CONCLUSION: Statin use has become more aggressive over time but there is still substantial room for improvement in dose adjustments and achieving target LDL levels.

INFLAMMATORY MARKERS, HEMOSTATIC FACTORS, AND THE ANKLE BRACHIAL INDEX. M.M. McDermott ${ }^{1}$, D. Green ${ }^{1}$, P. Greenland ${ }^{1}$, K. Liu ${ }^{1}$, M. Criqui ${ }^{2}$, C. Chan ${ }^{1}$, J.M. Guralnik $^{3}$, W.H. Pearce ${ }^{1}$, P.M. Ridker ${ }^{4}$, L. Taylor ${ }^{5}$, N. Rifai ${ }^{4}$, J.R. Schneider ${ }^{1}$, M. Quann ${ }^{1}$; ${ }^{1}$ Northwestern University, Chicago, IL; ${ }^{2}$ University of California at San Diego, San Diego, CA; ${ }^{3}$ National Institute on Aging, Bethesda, MD; ${ }^{4}$ Harvard Medical School, Boston, MA; ${ }^{5}$ Oregon Health and Science University, Portland, OR (Tracking ID \#74969)

BACKGROUND: We studied associations between blood markers and the ankle brachial index (ABI) in patients with and without clinically evident coronary or cerebrovascular disease. METHODS: Participants were 601 patients with and without peripheral arterial disease (PAD). We measured d-dimer, prothrombin 1.2, high-sensitivity C-reactive protein (CRP), fibrinogen, and serum amyloid A (SAA). Presence of cardiac or cerebrovascular disease was determined by combining data from medical record review, a primary care physician questionnaire, and patient report.

RESULTS: The table shows relationships between blood factors and the ABI among patients without known cardiac or cerebrovascular disease. In patients with cardiac or cerebrovascular disease, ABI was associated with d-dimer $(\mathrm{p}<0.001)$ but not other blood factors. In multivariable regression analyses of all participants adjusting for all blood factors, comorbid disease, and other confounders, only d-dimer was associated independently with ABI $(\mathrm{p}<0.001)$. CONCLUSION: D-dimer, a measure of ongoing thrombosis and fibrinolysis, may be more sensitive than other blood markers to atherosclerosis severity in leg arteries.

Blood factors and the ABI in Patients without Known Cardiac or Cerebrovascular Disease

$\begin{array}{lcccccc} & \text { ABI } & \begin{array}{c}\text { ABI } 0.50 \text { to } \\ <0.70(n=55)\end{array} & \begin{array}{c}\text { ABI } 0.70 \text { to } \\ <0.90(n=69)\end{array} & \begin{array}{c}\text { ABI } 0.90 \text { to } \\ <1.10(n=77)\end{array} & \begin{array}{c}\text { ABI } 1.10 \text { to } \\ <1.50(n=69)\end{array} & \text { P trend } \\ \text { D-dimer (ug/ml) } & 1.01 & 0.76 & 0.72 & 0.62 & 0.43 & <0.001 \\ \text { Prothrombin 1.2 (mg/dl) } & 1.32 & 1.11 & 1.16 & 1.02 & 0.95 & 0.001 \\ \text { Fibrinogen (mg/dl) } & 374 & 358 & 369 & 360 & 328 & 0.005 \\ \text { CRP (ug/dl) } & 0.56 & 0.44 & 0.52 & 0.53 & 0.25 & <0.001\end{array}$

READABILITY OF BROCHURES ON HUMAN IMMUNODEFICIENCY VIRUS INFECTION. E.L. McNeill ${ }^{1}$, C.A. Estrada ${ }^{1}$, T. Pinn ${ }^{1}$, C. Collins ${ }^{1}$, J.C. Byrd ${ }^{1} ;{ }^{1}$ East Carolina University, Greenville, NC (Tracking ID \#76501)

BACKGROUND: Functional health literacy is the ability to use reading and writing skills to follow medical instructions and to understand disease processes. Brochures and pamphlets are an inexpensive method of providing information about human immunodeficiency virus (HIV) infection. The readability of patient brochures is unknown.

PURPOSE: We assessed the readability of brochures available to patients with HIV infection. METHODS: We obtained printed patient information from state and federal governmental agencies, health advocacy groups, educational companies, pharmaceutical companies, and the Internet. Information written in languages other than English or for health care professionals was excluded. Fifty-one brochures were analyzed using a readability scale (SMOG). A grade level for each brochure was calculated.

RESULTS: The overall mean grade level for the brochures was 11.0. Five (9.8 \%) of the brochures were written on the eighth grade level or less. Eleven (22\%) of the brochures were written at the college level. Only educational companies, health advocacy groups, and governmental agencies had any written materials written at or below the eighth grade level. In our HIV clinic, $60 \%$ of patients' reading levels are below the ninth grade. 
CONCLUSION: Brochures intended to provide information about HIV infection are written primarily at the high school level. Most patients in our HIV clinic would be unable to comprehend the current brochures.

Table 1

\section{Brochure Category}

Educational Companies

Health Advocacy Groups

Governmental Agencies

Pharmaceutical Companies

Internet

$\begin{array}{cc}\text { Number (\%) } & \text { Mean Grade Level } \\ 17(33 \%) & 10.4 \\ 8(16 \%) & 10.9 \\ 11(21 \%) & 11.1 \\ 9(18 \%) & 11.7 \\ 6(12 \%) & 11.8\end{array}$

EARLY HEALTH IMPLICATIONS OF WEIGHT DEVELOPMENT IN YOUNG ADULTS. K.M. McTigue ${ }^{1}$, J.M. Garrett ${ }^{2} ;{ }^{1}$ University of Pittsburgh, Pittsburgh, PA; ${ }^{2}$ University of North Carolina at Chapel Hill, Chapel Hill, NC (Tracking ID \#76665)

BACKGROUND: Obesity is an important clinical and public health problem, with escalating prevalence, particularly in certain ethnic groups, and substantial health complications. While younger onset of obesity has been noted, early health implications of this trend are not well understood. We undertook this analysis to determine how the age at onset of obesity and the body mass index $\left(\mathrm{BMI}=\mathrm{kg} / \mathrm{m}^{2}\right)$ at age 21-24 years influence cardiovascular outcomes and quality of life at approximately age 40 .

METHODS: We analyzed data from 2706 men and women in the National Longitudinal Survey of Youth 1979 (NLSY79). Participants self-reported height, weight (13 weight queries from 1981 to 2000), and ethnicity (white, black or Hispanic), and, upon reaching age 40, answered a series of health questions. We used logistic regression to assess the relationship between the two measures of young adult weight dynamics and selected health outcomes. Cardiovascular outcomes were self-reported hypertension, diabetes, or high cholesterol diagnoses. Quality of life was measured by validated scales.

RESULTS: A 5-year delay in obesity onset was associated at age 40 with a $20 \%$ lower odds of hypertension in men (OR $0.80,95 \%$ CI, $0.66-0.97), 41 \%$ lower odds of hypertension in women (OR $0.59,95 \%$ CI $0.49-0.72$ ), $51 \%$ lower odds of diabetes in men (OR $0.49,95 \%$ CI, $0.34-0.71$ ), $53 \%$ lower odds of diabetes in women (OR 0.47, 95\% CI, 0.35-0.65), better physical functioning in women, and no change in mental functioning or odds of hyperlipidemia in men or women. A $5 \mathrm{~kg} / \mathrm{m}^{2}$ increase in BMI at age 21-24 was associated with hypertension in men (OR1.80, 95\% CI, 1.46-2.21) and women (OR 1.84, 95\% CI,1.57-2.16), with diabetes in men (OR 2.56, 95\% CI, 1.86-3.52) and women (OR 2.16, 95\% CI, 1.75-2.66), and with hyperlipidemia and impaired physical functioning in women. Both measures of weight dynamics conferred risk within 3 ethnic groups.

CONCLUSION: Younger onset of obesity and increasing BMI at age 21-24 are linked with adverse health effects at an early age. Understanding how early adult weight development influences health may be important for designing intervention. Clinical attention to excess weight in young adulthood has potential value in cardiovascular prevention.

FACTORS ASSOCIATED WITH "SHORT-TERM" AND "LONG-TERM" ADHERENCE TO ANTIRETROVIRAL THERAPY IN PATIENTS WITH HIVIAIDS. J.M. Mrus ${ }^{1}$, S.N. Sherman ${ }^{2}$ M.L. $\mathrm{Ho}^{2}$, R.W. Hornung ${ }^{2}$, J. Feinberg ${ }^{2}$, S.L. Fultz ${ }^{3}$, A.C. Justice ${ }^{3}$, C.M. Puchalski ${ }^{4}$ K.L. Mandell ${ }^{2}$, J. Tsevat ${ }^{1} ;{ }^{1}$ VAMC \& Univ. of Cincinnati, Cincinnati, OH; ${ }^{2}$ Univ. of Cincinnati, Cincinnati, OH; ${ }^{3}$ VA Pittsburgh Healthcare System, Pittsburgh, PA; ${ }^{4}$ George Washington Univ., Washington, DC (Tracking ID \#74239)

BACKGROUND: To report antiretroviral (ARV) adherence rates in a multicenter cohort and to evaluate factors associated with "short-term" (last 4 days) and "long-term" (last 6 months) adherence to ARV therapy in patients with HIV/AIDS.

METHODS: Self-reported adherence was assessed using a modified AIDS Clinical Trials Group measure in a cohort of 379 subjects with HIV/AIDS from 4 clinical sites in 3 cities in 2002. Adherence was evaluated using 2 distinct cut-offs: $100 \%$ adherent for at least the last 6 months and at least $95 \%$ adherent for the last 4 days. Covariates assessed for associations with adherence were: standard demographic characteristics; clinical characteristics; injection drug use (IDU) history (ever vs. never used); alcohol use (drinks per month); religiosity/spirituality; health status; depressive symptoms; symptom index (number of symptoms and degree of bother); social support; self esteem; optimism; and risk attitude. Univariate analyses were performed using the chi-square test or simple logistic regression. Multivariable analyses (including covariates with $\mathrm{p}$-values $\leq 0.25$ in univariate analyses) were performed using logistic regression.

RESULTS: $74 \%$ (281 of 379) of participants reported being on a stable ARV regimen for at least the last month. Of those patients, the mean (SD) age was 43.8 (8.6) and the mean CD4 cell count was 413 (286), $88 \%$ were male, $51 \%$ were white, $23 \%$ were veterans, $14 \%$ had remote IDU, and $2 \%$ had active IDU. 34\% (95 of 281) reported not missing any ARV doses in the last 6 months, and $73 \%$ (198 of 273 ) reported at least $95 \%$ adherence in the last 4 days. $38 \%$ of women vs. $33 \%$ of men and $33 \%$ of whites vs. $35 \%$ of non-whites reported not missing any ARV doses in the past 6 months $(\mathrm{p}=\mathrm{NS})$. In other univariate analyses, none of the covariates were significantly associated with "long-term" adherence and only age was significantly associated with "short-term" adherence (mean age of those that were adherent was 44.4 vs. 42.0 for those that were not adherent). In multivariable analyses, not working and having greater social support were significantly associated $(\mathrm{p}<0.05)$ with $100 \%$ adherence for at least the previous 6 months, and older age and a lower symptom index were significantly associated with at least $95 \%$ adherence in the past 4 days.

CONCLUSION: "Long-term" adherence was associated with not working and having greater social support, while "short-term" adherence was associated with older age and having few symptoms. These results imply that interventions that promote better symptom management and increase social support may improve adherence to ARV therapy. Given the general lack of significant association of sociodemographic characteristics with adherence, physicians should take care not to be overly influenced by those characteristics when forming their recommendations for treatment.

FACTORS INFLUENCING PATIENT ATTITUDE/PERCEPTIONS ABOUT ANTIRETROVIRAL THERAPY (ART). P. Clay ${ }^{1}$, D. Johnson ${ }^{2}$, R. Campo ${ }^{3}$, J. DeHovitz $^{4}$ K. Squires ${ }^{2}$, W. Jordan ${ }^{5}$, D. Thompson 6 , S. Narayanan ${ }^{7}$, S. Sajjan ${ }^{7}$, P. Duong ${ }^{7}$, L. Markson ${ }^{7}$; ${ }^{1}$ Kansas City Free Health Clinic, Kansas City, MO; ${ }^{2}$ USC, Los Angeles, CA; ${ }^{3}$ University of Miami, Miami, FL; ${ }^{4}$ SUNY Health Science Center, Brooklyn, NY; ${ }^{5}$ Oasis Clinic, Los Angeles, CA; ${ }^{6}$ Chase Brexton Health Services, Baltimore, MD; ${ }^{7}$ Merck \& Co., Inc., West Point, PA (Tracking ID \#74067)

BACKGROUND: Patient's (pt) attitude/perceptions about ART may influence the success of both short-term and long-term therapeutic management strategies.

METHODS: 304 pts participated in an observational study of adult HIV patients for whom a change in ART was being considered by their providers. Pts completed a questionnaire (Qr) that obtained information on demographics, HIV risk behaviors, pt worries, pt-provider communication (about anti-HIV medicines), trust, and knowledge of actual CD4 \& viral load (VL) counts. Pts were also asked about their attitude/perception about ART and its effect on: making them live longer (Q1), providing ability to fight off HIV/AIDS infection (Q2), improvement of HIV viral load (Q3) and CD4 count (Q4). Each of these 4 Questions were scored on a 5-point Likert scale (1: 'definitely no'; 5 : 'definitely yes'). The 4 questions were combined to a composite score of 0 (worse attitude) to 100 (better attitude). Factors associated with patient's better attitude/perception towards ART were ascertained through a linear regression model with the following variables: pt demographics, risk behaviors, depression, medication (med) experience, pt-provider communication/trust, worries, and pt knowledge of their CD4/VL counts.

RESULTS: Results: Pt demographics for 304 pts include: mean age $41 \mathrm{yrs} ; 31 \%$ female; $51 \%$ African American, 26\% Hispan-ics, 17\% Caucasians; 38\% had AIDS; 33\% had less than high school education; $67 \%$ had income below $\$ 15,000$. 57\% reported signs of depression \& $32 \%$ reported substance abuse in the past yr. In response to the questions on attitude/perceptions towards ART, $54 \%, 65 \%, 64 \%$ and $66 \%$ answered 'definitely yes' for Q1 through Q4 respectively. The mean composite score was 86.5 . Pts with less than high school education had lower score compared to those educated at high school level or more (83.3 vs 88.2; $\mathrm{p}=.03$ ); Pts with depression had lower scores compared to those without depression ( 84.9 vs $89.2 ; \mathrm{p}=.05)$. In the linear regression model, lower pt-provider communication ( $\mathrm{p}=<.01)$, lack of $\mathrm{pt}$ knowledge of CD4 and/or VL counts $(\mathrm{p}=<.01)$ and higher med worries $(\mathrm{p}=.02)$ were found to be associated with lower attitude/perception about ART.

CONCLUSION: Our results suggest that the level of communication between pt-provider about anti-HIV meds, pt knowledge of their CD4 and/or VL counts and medication worries are important factors influencing pt attitude/perception about ART.

IMPACT OF PATIENT'S HIV KNOWLEDGE ON MEDICATION ADHERENCE, GENERAL HEALTH PERCEPTION AND PATIENT WORRIES. W. Jordan ${ }^{1}$, J. DeHovitz ${ }^{2}$, R. Campo ${ }^{3}$ P.G. Clay $^{4}$, D. Johnson ${ }^{5}$, K. Squires ${ }^{5}$, D. Thompson ${ }^{6}$, S. Narayanan ${ }^{7}$, P. Duong ${ }^{7}$ L. Markson; ; ${ }^{7}$ Oasis Clinic, Los Angeles, CA; ${ }^{2}$ SUNY Health Science Center, Brooklyn, NY; ${ }^{3}$ University of Miami, Miami, FL; ${ }^{4}$ Kansas City Free Health Clinic, Kansas City, MO; ${ }^{5}$ USC, Los Angeles, CA; ${ }^{6}$ Chase Brexton Health Services, Baltimore, MD; ${ }^{7}$ Merck \& Co., Inc., West Point, PA (Tracking ID \#76375)

BACKGROUND: Limited information is available on the impact of patient's HIV knowledge on their worries, general health perception and medication adherence.

METHODS: HIV PRACTICE Cooperative is an observational study of adult HIV patients (pts). An 83-item questionnaire was administered to 304 pts for whom a change in ART was being considered by their providers. Data was captured on pt demographics, HIV risk behavior, health perception, HIV knowledge, attitudes, medication (med) and HIV related health worries, pt-provider communication/trust, and Med adherence (having missed at most one dose in past 3 days). This analysis focused on data of pt's HIV-related knowledge assessed through 3 questions: what are Anti-HIV meds?, what is CD4? \& what is viral load? Patients were classified as having answered correctly all 3 questions (group A), answered correctly 1 or 2 of 3 questions (group B), or no correct answer for any of the questions (group C). Pt demographics, knowledge, risk behaviors, worries, attitudes, pt-provider communication/trust variables were used in the logistic regression model to evaluate factors associated with med adherence.

RESULTS: Descriptive statistics are shown in the table below. In the logistic model to predict adherence, after adjusting for other parameters, pts with higher knowledge (Group A compared to $\mathrm{B} / \mathrm{C}$; OR: $2.53, \mathrm{p}=.03)$, Male pts (OR: 2.62, $\mathrm{p}=.03$ ) and pts with less med worries $(\mathrm{OR}=$ $1.05, \mathrm{p}<.01)$ were found more likely to adhere to meds, while pts with annual income $<\$ 15000$ $(\mathrm{OR}=0.37, \mathrm{p}=.04)$ were found less likely to adhere to meds.

CONCLUSION: Our findings suggest that, better HIV-related knowledge, higher income, less medication worries and male gender were associated with better med adherence.

\begin{tabular}{|c|c|c|c|c|}
\hline DESCRIPTIVE S TATIS TICS: & $\begin{array}{l}\text { All Pts } \\
(\mathrm{H}=304)\end{array}$ & $\begin{array}{l}\text { Group-A } \\
(\mathrm{N}=113\end{array}$ & $\begin{array}{l}\text { Group-B } \\
\text { QS }=160\end{array}$ & $\begin{array}{l}G \text { roup }-\mathrm{C} \\
(\mathrm{N}=25)\end{array}$ \\
\hline Female & $31 \%$ & $25 \%$ & $39 \%$ & $55 \%$ \\
\hline Pts with $<$ highschooleducation & $33 \%$ & $24 \%$ & $36 \%$ & $56 \%$ \\
\hline Pts with amoral income $<\$ 15000$ & $62 \%$ & $62 \%$ & $72 \%$ & $52 \%$ \\
\hline $\begin{array}{l}\text { Med a dherence (\% pts reporting - having missed at } \\
\text { most one dose inpast } 3 \text { days) }\end{array}$ & $70 \%$ & $79 \%$ & $67 \%$ & $52 \%$ \\
\hline General Health Perception S core (0 warst, 100 best) & 60.5 & 647 & 58.8 & 493 \\
\hline Medication womies Score (0 most, 100 least) & 76.4 & 728 & 76.4 & 693 \\
\hline HIV health-related womies $S$ cone $(0$ most 100 least $)$ & 66.4 & 70.5 & 650 & 55.4 \\
\hline Pt-Brovider commomication Score (0worst, 100 : best) & 84.3 & 84.1 & 840 & 821 \\
\hline
\end{tabular}


PREDICTING IMPAIRED GLUCOSE TOLERANCE USING COMMON CLINICAL INFORMATION-DATA FROM NHANES III. K. Nelson ${ }^{1}$, E.J. Boyko ${ }^{1}$; ${ }^{1}$ University of Washington, Seattle, WA (Tracking ID \#73646)

BACKGROUND: Recent clinical trials report that lifestyle modifications or pharmacotherapy among individuals with impaired glucose tolerance (IGT) can reduce their risk of developing diabetes. The purpose of this study is to develop a score to predict IGT using common clinical data.

METHODS: We analyzed data from the Third National Health and Nutrition Examination Survey (NHANES III) for 2746 individuals aged 40 to 74 who completed an oral glucose tolerance test. IGT was defined as a 2-hour post-challenge glucose of greater or equal to 140 $\mathrm{mg} / \mathrm{dL}[7.7 \mathrm{mmol} / \mathrm{L}]$. We performed bivariate and multivariate analyses to describe the association of IGT with commonly available clinical information. To assess the external validity of the full multivariate model, we used an internal bootstrap technique to estimate the area under the ROC curve for the predicted probability of IGT. A numerical score to predict IGT was derived from the results of the multivariate logistic regression models. RESULTS: Fasting glucose levels between $101-109 \mathrm{mg} / \mathrm{dL}$ [5.6-6.0 mmol/L] or between $110-125 \mathrm{mg} / \mathrm{dL}[6.1-6.9 \mathrm{mmol} / \mathrm{L}]$ were associated with IGT [OR 1.8 and 6.2 respectively, $\mathrm{p}<0.05$ ]. Body Mass Index (BMI) of greater than or equal to $25 \mathrm{~kg} / \mathrm{m} 2$, Mexican American ethnicity, age between 60 and 74 years, hypertension and triglyceride level greater or equal to $150 \mathrm{mg} / \mathrm{dL}[1.69 \mathrm{mmol} / \mathrm{L}]$ were also associated with IGT. The regression model was reliable (goodness-of-fit test, $\mathrm{p}=0.11$ ) and discriminated well between individuals with IGT and those without IGT (area under the ROC curve, 0.76). The area under the receiver-operating characteristic (ROC) curve for an 8 point scale derived from the multivariate analysis was 0.74 $(95 \%$ CI $0.72,0.76)$. Setting a low cut point of 2 on this scale resulted in high sensitivity $(86 \%)$, while a high cut point of 6 yielded high specificity (97\%) for the detection of IGT.

CONCLUSION: A numerical score based on common clinical data can identify individuals with a low or high likelihood of having IGT.

SELF-PERCEPTION OF WEIGHT AMONG A NATIONAL SAMPLE OF OVERWEIGHT AND OBESE AMERICANS. K. Nelson ${ }^{1}, \mathrm{~K}$. Bradley ${ }^{1} ;{ }^{1}$ University of Washington, VA Puget Sound, Seattle, WA (Tracking ID \#73706)

BACKGROUND: Although the prevalence of obesity in the US is increasing, little is known about overweight and obese individuals' self-perception of weight. The purpose of this study was to evaluate overweight and obese individuals' self-perception of health, weight and weight loss practices using nationally representative data.

METHODS: We analyzed data from the Third National Health and Nutrition Examination Survey (NHANES III) conducted between 1988 and 1994 at 89 mobile examination centers. Adults older than 17 years who had a measured body mass index (BMI) calculated from standing weight and height were included in the analysis $(\mathrm{n}=18,099)$. Weight categories were defined as normal weight [BMI $<25 \mathrm{~kg} / \mathrm{m}^{2}$ ], overweight [BMI $25.0-29.9 \mathrm{~kg} / \mathrm{m}^{2}$ ], and obese (class 1 [BMI $30.0-34.9 \mathrm{~kg} / \mathrm{m}^{2}$ ], class 2 [BMI $\left.35.0-39.9 \mathrm{~kg} / \mathrm{m}^{2}\right]$, and class 3 [BMI $>40.0 \mathrm{~kg} /$ $\left.\mathrm{m}^{2}\right]$ ). Bivariate analyses were used to describe self-perception of weight, desire to loose weight and weight loss attempts by weight category. Multivariate logistic analyses were used to identify individual characteristics associated with obesity.

RESULTS: Fifty three percent of US adults were overweight $(31 \%)$ or obese $(14 \%$ class 1 ; $5 \%$ class $2 ; 3 \%$ class 3 ). While $33 \%$ of overweight adults did not consider themselves overweight and $26 \%$ did not want to weigh less, $45 \%$ reported trying to loose weight in the past year. Almost all obese individuals (class 1, 2, and 3) considered themselves overweight $(90 \%, 97 \%$, and $98 \%$ respectively) and would like to weigh less $(92 \%, 97 \%$ and $99 \%$ respectively), and a majority had tried to loose weight in the past 12 months $(61 \%, 70 \%$ and $73 \%$ respectively). One third of obese individuals reported changing their eating habits in the past 12 months for medical reasons and $65 \%$ reported being less active than 10 years earlier. Individuals who were obese were more likely to report fair or poor health and were more likely to be women, African American and Mexican American, and have less than a high school education $(\mathrm{p}<0.001)$.

CONCLUSION: The vast majority of obese individuals consider themselves overweight and would like to weigh less. However, only one third of obese individuals had tried to change their eating habits in the past year and many reported less physical activity than 10 years earlier. Many overweight individuals are not aware of their weight problem and may represent an important group for health interventions.

VALIDATION OF PATIENT SELF-REPORT OF DIABETES MELLITUS. J. Nquyen ${ }^{1}$ M.B. McDonell ${ }^{2}$, B.A. Young ${ }^{3}$, S.D. Fihn ${ }^{3} ;{ }^{1}$ University of Washington School of Medicine, Seattle, WA; ${ }^{2}$ VA Puget Sound Health Care System, Seattle, WA; ${ }^{3}$ University of Washington Department of Medicine, VA Puget Sound Health Care System, Seattle, WA (Tracking ID \#75871)

BACKGROUND: Limited research has been done to validate the accuracy of self-report of chronic disease where computerized administrative databases are used to extract clinical information. Our objective was to validate the accuracy of self-reported diabetes among Veteran Affairs (VA) patients.

METHODS: We conducted a cross-sectional analysis of baseline data collected for the Ambulatory Care QUality Improvement Project (ACQUIP). Eligible participants were sent a "Health Checklist" that identified common chronic medical conditions. Seattle respondents for whom laboratory, clinical data and pharmacy data were available were selected for the validation study. Diabetes was defined by self-report (SR) or by having one or more of the following present: an ICD-9 code for diabetes (250.XX), medial record diabetes diagnosis, diabetic medication prescription (insulin or oral hypoglycemic agents), or diabetes specific laboratory data (fasting plasma glucose $>126 \mathrm{mg} / \mathrm{dL}$, random glucose $>200 \mathrm{mg} / \mathrm{dL}$ or HbAlc $>7.0 \%$ ). Using ICD-9 codes as the gold standard, validity of SR was assessed.

RESULTS: Of the 90,062 veterans enrolled in ACQUIP, screening questionnaires were sent to 11,392 Seattle VA patients. Of those, $6,995(61 \%)$ returned the health checklist; diabetes SR occurred in $1625(23.2 \%)$. For diabetic respondents, the positive predictive value of SR was $87.8 \%$ compared to ICD-9 code. Compared to those for whom diabetes was validated (true positives [TP]), those for whom diabetes was not validated (false positives $[\mathrm{FP}]$ and false negatives [FN]) had similar demographic characteristics but had a higher proportion of surveys completed by proxy, more comorbid conditions and a higher proportion with hemoglobin A1c $>8 \%$. Determination of the kappa statistic between SR and ICD-9 diagnosis of diabetes was $0.84(\mathrm{p}<0.001)$. Use of a laboratory test $(\mathrm{k}=0.73, \mathrm{p}<0.001)$ or diabetic prescriptions $(\mathrm{k}=0.82$, $\mathrm{p}<0.001)$ instead of SR decreased the kappa slightly. Addition of diabetic medications $(\mathrm{k}=$ $0.84, \mathrm{p}<0.001)$, laboratory tests $(\mathrm{k}=0.78, \mathrm{p}<0.001)$ or the combination of the two $(\mathrm{k}=0.78$, $\mathrm{p}<0.001$ ), did not improve the kappa.

CONCLUSION: SR of diabetes has excellent correlation with clinical diagnosis of diabetes. Addition of laboratory or medication data may help to ascertain false positives.

CHARACTERISTICS RELATED TO INCREASED CHRONIC NON-MALIGNANT PAIN PERCEPTION. J. O'Rorke ${ }^{1}$, J. Jarvis ${ }^{2} ;{ }^{1}$ University of Texas Health Science Center at San Antonio, San Antonio, TX; ${ }^{2}$ University of Louisville, Louisville, KY (Tracking ID \#74403)

BACKGROUND: The Joint Commission on Accreditation of Healthcare Organizations (JCAHO) and other organizations have emphasized the importance of physician awareness of patients who suffer chronic pain. This study was designed to measure pain in a group of these patients and to identify associations with worsening perceptions of pain amenable to intervention.

METHODS: Patients, with $>3$ months of pain, presenting to primary care clinics at 12 U.S. institutions, completed a verbally administered survey. After completion of the survey and visit with their doctor, their doctor completed a survey about the patient's pain. Bivariate analyses examined associations between patient characteristics, co-morbid illness, use of complimentary therapies, pain clinic utilization, and pain perception as interpreted by both patient and MD. A multivariate analysis of significant data $(\mathrm{p}<0.2)$ was performed to control for potential confounders.

RESULTS: Data were based on 345 patient and physician surveys. The mean patient age was 53 years (23-87). Sixty-eight percent were female and $45 \%$ were white. Participants averaged 3 co-morbid diseases and the mean pain perception on a pain scale from $0-10$ was 6.7 (st.dev $=$ 2.4). Bivariate analyses demonstrated a relationship between the following variables and the patient's pain rating:

Pt-physician discordance in pain rating Corr $=.61$

Sees same doctor at visits(continuity) $\quad \mathrm{P}<.004$

Depression

Pt feels disabled from pain

Seen by pain clinic/specialist

Race (black vs. white)

$\mathrm{P}<.0000$

$\mathrm{P}<.005$

$\mathrm{P}<.03$

$\mathrm{P}<.006$

Physical Therapy $\quad \mathrm{P}<.02$

In the linear regression, perception of pain was associated with discordance in pain rating between physician and patient, use of a pain clinic, use of alternative medicine, concomitant depression, discontinuity of care, and patient feelings of disability due to pain $(\mathrm{P}<.05)$. The R-squared equaled 44 .

CONCLUSION: These results point to several potentially reversible components of more severe chronic pain. For example, depression is amenable to therapy, discordance could be addressed through enhanced communication, and continuity of care could be prioritized. The actual effect of such interventions of pain scores should be studied.

CHANGES IN RATES OF BETA- BLOCKER USE IN COMMUNITY HOSPITAL PATIENTS WITH ACUTE MYOCARDIAL INFARCTION. A.B. Olomu ${ }^{1}$, R.E. Watson ${ }^{1}$, A. Siddiqi ${ }^{1}$, M.M. Holmes-Rovner ${ }^{1}$, F.C. Dwamena ${ }^{1}$, B.A. Mclntosh ${ }^{2}$, J. Kupersmith ${ }^{1} ;{ }^{1}$ Michigan State University, East Lansing, Ml; ${ }^{2}$ Hurley Medical Center, Flint, Ml; ${ }^{3}$ Texas Tech School of Medicine, Lubbock, TX (Tracking ID \#76047)

BACKGROUND: Despite the overwhelming evidence from several randomized controlled trials that beta-blocker (BB) therapy for acute myocardial infarction (AMI) decreases mortality and reinfarction, studies have repeatedly demonstrated under-use of BB. Like previous studies, our group, the Michigan State University Institutional Collaborative Heart (MICH) Study Group, 1994-1995 (Phase 1) found underutilization of BB in patients following AMI in five mid-Michigan community hospitals. Since this initial study, several initiatives, including the Michigan Peer Review Organization (MPRO), were set up to improve the quality of care and use of appropriate evidenced based medications following AMI. The objective of this study was to determine if rate of $\mathrm{BB}$ use on admission, in hospital, and at discharge increased between Phase 1 and Phase II (1997) in patients with AMI.

METHODS: We enrolled and reviewed the charts of all patients prospectively identified with AMI in Phase $\mathrm{I}(\mathrm{n}=1,163)$ and in Phase II $(\mathrm{n}=508)$ in five mid-Michigan community hospitals. Exclusion criteria included chronic obstructive pulmonary disease, congestive heart failure, left ventricular ejection fraction $<35$, complete heart block, pulse $<60$, and use during the hospitalization of an intraaortic balloon pump or pressors for hypotension. We compared the use of $\mathrm{BB}$ in the two cohorts.

RESULTS: Prescription of BB to ideal patients with AMI increased in-hospital (53.8 \% vs. $72.8 \% ; \mathrm{p}<0.001)$ and at discharge $(33.6 \%$ vs. $61.7 \% ; \mathrm{p}<0.001)$. No change was observed on admission among patients with previous AMI (25.9 vs. $25 \%$ p $>0.40)$. Logistic regression analysis did not identify race or sex as predictors of BB use. Younger age predicted BB use inhospital (OR 8.26, 95\% CI 1.85-36.75) and at discharge. (OR 2.84, 95\% CI 1.11-7.26). CONCLUSION: BB use in-hospital and at discharge improved over the study period. However, improvement to $100 \%$ should be achieved in these ideal BB candidates. Lack of improvement in secondary prevention is suggested by no change in use among patient with previous AMI on arrival to the hospital. There still remains the need to improve the use of this relatively inexpensive but efficacious modality. Initiatives to improve use of BB after discharge and among elderly patients with AMI are urgently needed. 
IMPROVING HOSPITAL DISCHARGE FOR ELDERLY PATIENTS WITH CHF. C. Phillips ${ }^{1}$, S.M. Wright ${ }^{1}$, D.E. Kern ${ }^{1}$, R.M. Singa ${ }^{1}$, S. Shepperd ${ }^{2}$, H.R. Rubin ${ }^{1}$; ${ }^{1}$ Johns Hopkins University, Baltimore, MD; ${ }^{2}$ Institute of Health Sciences, Oxford University, Headington, Oxford (Tracking ID \#76886)

BACKGROUND: Readmission rates among elderly patients with congestive heart failure (CHF) may be reduced by comprehensive discharge planning plus post-discharge support. The aim of this review is to evaluate the effect of comprehensive discharge planning plus postdischarge support versus usual care on readmission rates, mortality, initial length of stay (LOS), quality of life scores, and medical costs.

METHODS: We searched MEDLINE, the Cochrane Clinical Trials Register and other databases for English language publications of randomized controlled trials among patients with $\mathrm{CHF}$, mean age $>60$, comparing comprehensive discharge planning plus post-discharge support with usual care and reporting any of the listed outcomes. Two authors independently reviewed each report, assigned quality scores and recorded results. Pooled differences are reported as relative risk using a random effects model or mean values.

RESULTS: We found 17 eligible trials including 3141 patients with CHF. Compared with patients receiving usual care, patients receiving comprehensive discharge planning plus postdischarge support had fewer readmissions (relative risk $\{R R\} 0.74$ [95\% Confidence Interval (CI) $0.64,0.86] \mathrm{N}=17$ studies); similar mortality RR 0.81 [95\% CI $0.62,1.05] \mathrm{N}=11$ ); similar mean $\operatorname{LOS}(8.9+2.5$ vs. $8.8+2.0$ days $[95 \% \mathrm{CI}$ of difference -0.10 to 0.10$] \mathrm{N}=9)$, greater increase in quality of life scores $(9$ vs. 17 percent (range $0-100), p<0.05(\mathrm{~N}=6)$; and no significant difference in mean medical costs $(\$ 9,900$ vs $\$ 7,600 \mathrm{p}>0.05, \mathrm{~N}=7)$, respectively. CONCLUSION: Comprehensive discharge planning plus post-discharge reduced readmission rates and may improve quality of life without increasing medical costs for elderly patients with CHF.

DIMENSIONS OF PATIENT-PROVIDER COMMUNICATION AND DIABETES SELFCARE IN AN ETHNICALLY-DIVERSE POPULATION. J.D. Piette ${ }^{1}$, D. Schillinger ${ }^{2}$, M.B. Potter $^{2}$, M. Heisler ${ }^{1} ;{ }^{1}$ Ann Arbor VAMC and the University of Michigan, Ann Arbor, Ml; ${ }^{2}$ University of California, San Francisco, San Francisco, CA (Tracking ID \#73886)

BACKGROUND: Patient-provider communication is essential for effective care of diabetes and other chronic illnesses. However, the relative impact of general versus disease-specific communication on self-management is poorly understood, as are the determinants of these two communication dimensions. The purpose of this study was to examine (1) the extent to which patient characteristics, provider characteristics, and system of care differences influence general and diabetes-specific communication; and (2) the importance of each communication dimension as a determinant of diabetes patients' self-care behavior.

METHODS: 752 diabetes patients were enrolled from three VA-, one county-, and one university-based health care system and completed a detailed telephone interview about their interactions with clinicians, self-care, health status, and service use. Patients' assessments of providers' general and diabetes-specific communication were measured using previously validated scales. Self-reported foot care; and adherence to hypoglycemic medications, dietary recommendations, and exercise were measured using standard items. OLS regression models were fit in order to identify independent patient, provider, and system-level predictors for each communication dimension, controlling for patients' clinical characteristics (A1C levels, insulin use, and history of cardiovascular illnesses) and the clustering of patients by primary care provider. Effects of communication on self-care were estimated by using multivariate, ordinal logit models to calculate the predicted probability of adequate self-care in each area among patients with varying general and diabetes-specific communication reports.

RESULTS: Fifty-two percent of participants were non-White, $18 \%$ had less than a highschool education, and $8 \%$ were primarily Spanish-speaking. General- and diabetes-specific communication reports were only moderately correlated $(r=.35, p<.0001)$. Sociodemographically-vulnerable patients (i.e., racial and language minorities and those with less education) reported communication that was as good or better than that reported by other patients. Patients receiving most of their diabetes care from their primary provider and patients with a longer primary care relationship reported better general communication (both $\mathrm{p}<.001$ ). VA- and county-clinic patients reported better diabetes-specific communication than university-clinic patients $(\mathrm{p}<.0001)$. In multivariate probit analyses, both dimensions of communication were independently associated with self-care in each of the four areas examined (all $\mathrm{p}<.05$ ). For example, patients with both poor general and diabetes-specific communication had a $75 \%$ predicted probability of adhering to hypoglycemic medications as compared to $97 \%$ among patients with the best communication in both dimensions. The probability of daily exercise increased from $12 \%$ to $42 \%$ when comparing patients with the worst and best communication reports.

CONCLUSION: General and diabetes-specific communication are related but unique domains, and improving either one may improve self-management. Providers in these sites are communicating successfully with vulnerable patients. Continuity of diabetes care (as indicated by a central role for the patient's primary care provider and a longer primary care relationship) may increase familiarity with patients' unique needs and the effectiveness of communication. At the system level, physicians in sites that see a higher volume of chronically-ill and sociodemographically vulnerable patients, such as VA and county clinics, may become more effective at supporting meaningful interactions.

CLAIMS DATA MEASUREMENTS OF MEDICATION ADHERENCE AND METABOLIC OUTCOMES IN PATIENTS WITH TYPE II DIABETES. M. Pladevall ${ }^{1}, \mathrm{~K}$. Williams ${ }^{1}, \mathrm{H} . \mathrm{Xi}^{1}$, J. Elston Lafata ${ }^{1} ;{ }^{1}$ Henry Ford Health System, Detroit, MI (Tracking ID \#76156)

BACKGROUND: Non-adherence to medications is a common problem in clinical practice especially among patients with asymptomatic chronic conditions. Adherence is not routinely measured in clinical practice and a gold standard is not available. Yet, claims data are a potentially useful source of adherence information. No previous studies have examined the correlation between adherence and clinical outcomes among patients with Type II Diabetes (DMII).
METHODS: Using automated data sources available within a large health system, we identified patients with DMII who were continuously enrolled in the health plan and treated with metformin during the period 1999-2001. Among patients who met these criteria, we selected a random sample of 663 patients among whom information on both adherence to metformin, and glycosylated hemoglobin (HbAlc) and low density lipoprotein (LDL) levels, were measured. In the subset of patients who also were being treated with lipid lowering drugs, adherence to lipid medication was measured. Adherence was measured as 1-(the ratio of the number of gaps in days supplied/the number of days of observation). Mean and median adherence levels were estimated, and Wilcoxon and Spearman Correlation tests were used to assess the association of adherence to clinical outcomes. Linear regression models were used to adjust for socio-demographic characteristics.

RESULTS: As shown in the table, adherence was significantly associated with clinical outcomes. Adjustment for socio-demographic characteristics did not attenuate the strength of the associations.

CONCLUSION: Non-adherence was statistically associated with worse metabolic outcomes. Adherence measured by claims data correlates with clinical outcomes in DMII patients. Claims data is a valid and inexpensive method to measure adherence and could be useful in clinical practice.

\begin{tabular}{|c|c|c|c|c|}
\hline & \multicolumn{2}{|c|}{$\begin{array}{c}\text { Metformin sample } \\
\qquad \mathrm{N}=663\end{array}$} & \multicolumn{2}{|c|}{$\begin{array}{c}\text { Metformin and } \\
\text { lipid agents sample } \\
\mathrm{N}=258\end{array}$} \\
\hline Mean adherence & \multicolumn{2}{|c|}{$77.8 \%$} & \multicolumn{2}{|c|}{$83.1 \%$} \\
\hline Median adherence & \multirow{2}{*}{\multicolumn{2}{|c|}{$83.8 \%$}} & \multirow{2}{*}{\multicolumn{2}{|c|}{$\begin{array}{l}90.4 \% \\
41.0 \%\end{array}$}} \\
\hline Percent with adherence $<80 \%$ & & & & \\
\hline Adherence $<80 \%$ & $\frac{\text { Mean } \mathrm{HbAlc}}{8.7^{1}}$ & $\frac{\text { Mean LDL }}{115.9^{1}}$ & $\frac{\text { Mean } \mathrm{HbAlc}}{8.8^{1}}$ & $\frac{\text { Mean LDL }}{120.4^{1}}$ \\
\hline Adherence $\geq 80 \%$ & 7.8 & 107.9 & 8.2 & 105.0 \\
\hline Spearman's correlation & $0.24^{1}$ & $0.15^{1}$ & $0.17^{1}$ & $0.25^{1}$ \\
\hline
\end{tabular}

ALCOHOL AND CORONARY ARTERY CALCIFICATION: UNANTICIPATED FINDINGS IN CARDIA. M.J. Pletcher ${ }^{1}$, P. Varosy ${ }^{1}$, C.I. Kiefe ${ }^{2}$, C.E. Lewis ${ }^{2}$, S. Sidney ${ }^{3}$, S.B. Hulley ${ }^{1}$; ${ }^{1}$ University of California, San Francisco, San Francisco, CA; ${ }^{2}$ University of Alabama at Birmingham, Birmingham, AL; ${ }^{3}$ Division of Research, Kaiser Permanente, Oakland, CA (Tracking ID \#73747)

BACKGROUND: Many studies suggest that people who report moderate consumption of alcohol (1-2 drinks/day) have lower coronary heart disease event rates than those who report none. This association may represent a protective effect of alcohol against atherosclerosis, protection against some other process causing acute coronary events (thrombosis, inflammation), or confounding by social correlates of alcohol use. To clarify these relationships, we measured the association between alcohol consumption and coronary calcification, a surrogate for atherosclerosis.

METHODS: We used cross-sectional data collected in year 15 of CARDIA, a prospective study of African-American and Caucasian women and men aged 18-30 years at enrollment in 1985-1986. We compared the prevalence of coronary calcification in persons consuming different quantities of alcohol, and used logistic regression analysis to adjust for possible confounders (age, gender, race, smoking, income, education, and physical activity) and possible mediators of coronary artery disease (blood pressure, lipids, diabetes, body mass index, C-reactive protein, and fibrinogen).

RESULTS: The 3037 CARDIA participants with coronary calcium measurements were on average $40.3 \pm 3.6$ years old, $45 \%$ female, and $45 \%$ African-American. Alcohol consumption was light to moderate (see Table). There was a strong, direct association between the amount of alcohol consumed and the prevalence of coronary calcification, even after adjustment for possible confounders and mediators of coronary artery disease (see Table). Adjusting for confounders but not mediators yielded similar results. Race/sex specific analyses yielded no additional findings.

CONCLUSION: The association we observed between moderate alcohol consumption and coronary calcium does not support a protective effect on this outcome, and in fact suggests the opposite.

Prevalence of coronary calcification (CC) according to alcohol consumption (in drinks/day), crude and adjusted

$\begin{array}{lcccc} & \text { None } & <1 & 1-1.9 & \mathbf{2 +} \\ \mathrm{N}(\%) & 1435(47 \%) & 1023(34 \%) & 341(11 \%) & 238(8 \%) \\ \text { CC prevalence } & 8 \% & 9 \% & 13 \% & 19 \% \\ \text { OR-crude* }^{*} & 1(\mathrm{ref}) & 1.2(0.9-1.6) & 1.9(1.3-2.7) & 2.8(1.9-4.1) \\ \text { OR-adj. }^{8} & 1(\mathrm{ref}) & 1.1(0.8-1.5) & 1.4(0.9-2.2) & 1.8(1.2-2.9)\end{array}$

$\left({ }^{*} p<0.001\right.$ for trend; $\S p=0.004$ for trend $)$

SELF-RECOGNITION OF OBESITY STATUS AND WEIGHT LOSS ATTEMPTS IN A NATIONALLY REPRESENTATIVE SAMPLE OF OVERWEIGHT AND OBESE ADULTS. M.E. Poston ${ }^{1}$, L.E. Egede ${ }^{1} ;{ }^{1}$ Medical University of South Carolina, Charleston, SC (Tracking ID \#76572)

BACKGROUND: Obesity is a strong risk factor for cardiovascular disease (CVD) and allcause mortality. Long term success at weight loss is seldom achieved. This study used the 
Third National Health and Nutrition Examination Survey (NHANES III) to determine the relationship between self-recognition of obesity and prior attempts at weight loss. METHODS: 20,050 adults were surveyed in NHANES III. Data on 10,556 adults with body mass index $(\mathrm{BMI}) \geq 25$ were analyzed. Self-recognition of obesity was defined as selfclassification as "overweight" by people with BMI $\geq 25$. Prior weight loss attempt was defined by self-report of a weight loss attempt within the past 12 months. CVD-related comorbidities were defined as self-report of a physician diagnosed condition including diabetes mellitus, hypertension, high cholesterol, congestive heart failure, myocardial infarction or stroke. Number of comorbidities was categorized as none, 1,2 , and $\geq 3$. The transtheoretical model of change (TTMC) was the theoretical framework of the study. We defined "contemplation" as self-recognition of obesity and "action" as prior weight loss attempt. We determined the proportion of patients with self-recognition of obesity and prior weight loss attempts across covariates (sex, age, race, education, income, employment status, marital status, health insurance status, perceived health status, BMI and number of CVD comorbidities) using $\chi^{2}$ statistics. We used multiple logistic regression to determine the independent association between self-recognition of obesity and prior weight loss attempt while controlling for covariates. We used STATA to account for the complex survey design of NHANES III.

RESULTS: $66 \%$ of overweight patients and $92 \%$ of obese patients correctly classified themselves as overweight. $45 \%$ of overweight patients and $64 \%$ of obese patients reported prior weight loss attempts. $62 \%$ of patients with self-recognition of obesity reported prior weight loss attempts compared with $21 \%$ of patients without recognition of obesity $(\mathrm{p}<0.0001)$. After controlling for covariates, self-recognition of obesity was independently associated with prior weight loss attempts (OR 4.86, 95\% CI 3.66-6.45).

CONCLUSION: Self-recognition of obesity is independently associated with prior weight loss attempts. Consistent with the constructs of the TTMC, "contemplation" is associated with increased likelihood of "action". Internists should be aware of the importance of patient acceptance of being overweight in weight loss counseling sessions. Further studies are needed to determine what drives patient awareness of obesity.

IMPROVING CHF OUTCOMES USING AN IVR SYSTEM AND TARGETED NURSE FOLLOW-UP. D. Roblin ${ }^{1}$, C.S. Gardner ${ }^{1}$, M.G. Kawamura ${ }^{1}$, T. Washington ${ }^{1}$, J. Hinchman ${ }^{1}$; ${ }^{1}$ Kaiser Permanente, Atlanta, GA (Tracking ID \#76803)

BACKGROUND: Ineffective patient self-management, lack of knowledge of signs of worsening $\mathrm{CHF}$, and consequently acute $\mathrm{CHF}$ exacerbations contribute to CHF-related morbidity and costs. We developed an interactive voice recognition (IVR) system so that patients could transfer CHF diary information into a central database which was regularly profiled. Patients with signs of worsening CHF were contacted by a nurse care manager for assessment and follow-up. Patients could listen to and request printed copies of CHF-related health information. The principal objective of this study was to assess the impact of this disease management program on patient self-management, knowledge, quality of life (QoL), and risk of acute CHF exacerbations.

METHODS: CHF patients 30 years of age and older as of September $2001(\mathrm{~N}=728)$ were invited to participate. 106 patients consented and were randomized into a "telecare" group $(\mathrm{N}=64)$ and a usual care group $(\mathrm{N}=42)$. On study entry and exit (6-months later), patients were surveyed by. Multi-item scales for $\mathrm{CHF}$ self-management practices, knowledge of signs of worsening $\mathrm{CHF}$, frequency of CHF symptoms, and QoL were computed from survey responses. ED visits or hospital admissions indicative of worsening CHF (e.g. SOB, painful respiration) for the 6-month periods preceding and following study entry dates were collected from computerized databases.

RESULTS: $47 \%$ of the study population was 65 years or older; $48 \%$ were female. At baseline, telecare and usual care groups did not differ (alpha $=0.05)$ in demographic or clinical characteristics, frequency of CHF symptoms, knowledge of CHF symptoms, self-management practices, QoL, or rate of acute exacerbation in the prior 6-months. Compared with usual care, the telecare group significantly improved CHF self-management practices $(\mathrm{p}=0.02)$; however, neither improvement in knowledge of CHF symptoms or frequency of CHF symptoms nor reduction in acute exacerbations over the 6-month study period were obtained. Compared with usual care, compliant telecare patients $(\mathrm{N}=27)$ realized significant improvements in both CHF self-management practices $(\mathrm{p}=0.01)$ and some improvement in knowledge of symptoms of worsening CHF $(\mathrm{p}=0.09)$. Compared with noncompliant telecare patients, compliant telecare patients also achieved reduction in risk of acute CHF exacerbations $(\mathrm{p}=0.04)$ in the 6-month intervention period relative to the 6-month period prior to study entry.

CONCLUSION: Compared with usual care, telecare patients only improved CHF selfmanagement practices over a 6-month period. Compliant telecare patients obtained additional benefits of improved knowledge of symptoms of worsening CHF and reduction in risk of acute CHF exacerbations. Telecare is an effective complement to usual CHF care for changing patient behavior and outcomes among patients who comply with the intervention protocol.

A PRIMARY CARE-BASED, PHARMACIST-LED, DISEASE MANAGEMENT PROGRAM IMPROVES OUTCOMES FOR PATIENTS WITH DIABETES: A RANDOMIZED CONTROLLED TRIAL. R.L. Rothman ${ }^{1}$, M.P. Pignone ${ }^{2}$, R. Malone ${ }^{2}$, B. Bryant ${ }^{2}$, D.A. DeWalt ${ }^{2}$, B. Crigler ${ }^{2}$; 1 Vanderbilt Center for Health Services Research, Nashville, TN; ${ }^{2}$ University of North Carolina, Chapel Hill, NC (Tracking ID \#74196)

BACKGROUND: Disease management programs improve outcomes for patients with Type 2 diabetes. Few programs however, have (1) focused on patients with significant barriers to care, (2) used clinical pharmacists to provide care, and (3) addressed cardiovascular risk reduction. METHODS: We performed a randomized controlled trial of a pharmacist-led, diabetes disease management program for patients with poor glucose control. Adult patients, followed for their diabetes care in our general medicine practice, who had $\mathrm{HbA1C} \geq 8.0 \%$ were eligible. Participating patients were randomized to the intervention or usual care. The intervention includes (1) one-on-one educational sessions with a clinical pharmacist; (2) evidence-based treatment algorithms and a proactive computer system to manage glucose, blood pressure, cholesterol and aspirin use; and (3) frequent phone follow-up. Control patients received a onehour education session from the pharmacist, and usual care from their primary provider. Outcomes include HbA1c, blood pressure, lipid levels, and aspirin use at 6 and 12 months. RESULTS: From February 2001 to April 2002, we enrolled and randomized 218 patients. All patients have completed 6-month follow-up and complete data are available for 206 (94\%). To date, $152(70 \%)$ patients have completed 12 -month follow-up. Mean age is 55 years, $56 \%$ are female, $65 \%$ African American, and $72 \%$ report income under \$20,000 per year. Mean duration of diabetes is 8.4 years. At baseline, average $\mathrm{HbAl} \mathrm{c}$ was $10.8 \%$ and systolic blood pressure (SBP) was 139 . At 6 month follow-up, HbA1c improved by $2.6 \%$ points in the intervention group compared to $1.9 \%$ points in the control group (difference $0.7 \%$ points, $95 \% \mathrm{CI}-0.08,1.51$ ); SBP decreased by $6.6 \mathrm{mmHg}$ in intervention patients compared to a $3.5 \mathrm{~mm} \mathrm{Hg}$ increase in controls (difference $10.2 \mathrm{mmHg}, 95 \% \mathrm{CI} 3.2,17.1$ ), and $81 \%$ of intervention patients were on aspirin for cardiovascular risk reduction, compared to $55 \%$ of control patients $(\mathrm{p}<0.001)$. At 12 month follow-up to date: HbA1c improved by $2.3 \%$ points in the intervention group compared to $1.5 \%$ points in the control group (difference $0.8 \%$ points, $95 \%$ CI $-0.09,1.73$ ); SBP decreased by $4.9 \mathrm{mmHg}$ in intervention patients compared to an increase of $2.2 \mathrm{~mm} \mathrm{Hg}$ in controls (difference $7.1 \mathrm{~mm} \mathrm{Hg}, 95 \% \mathrm{CI}-0.5,14.8$ ), Total cholesterol/HDL ratio improved by 0.7 compared to 0.3 in the control group (difference $0.4,95 \% \mathrm{CI}-0.1,1.0$ ), and $79 \%$ of intervention patients were on aspirin compared to $55 \%$ of control patients $(\mathrm{p}<0.01)$.

CONCLUSION: A primary care-based, pharmacist-led disease management intervention is effective in improving clinical outcomes and reducing cardiovascular risk in patients with diabetes.

A LONGITUDINAL ANALYSIS OF THE RELATIONSHIP BETWEEN LITERACY AND METABOLIC CONTROL IN PATIENTS WITH DIABETES. R.L. Rothman ${ }^{1}$, M.P. Pignone ${ }^{2}$, R. Malone ${ }^{2}$, B. Bryant ${ }^{2}$, D.A. DeWalt ${ }^{2}$, B. Crigler ${ }^{1} ;{ }^{1}$ Vanderbilt Center for Health Services Research, Nashville, TN; ${ }^{2}$ University of North Carolina, Chapel Hill, NC (Tracking ID \#76148)

BACKGROUND: Inadequate literacy affects over 90 million Americans, and low literacy is associated with poor metabolic control in patients with diabetes. However, no previous studies have examined the relationship of literacy and glycemic control in a prospective manner.

METHODS: We sought to examine the impact of literacy on glycemic control in a cohort of patients enrolled in a randomized controlled trial of a comprehensive diabetes management program. Patients with poor glucose control $(\mathrm{HbAlc} \geq 8.0 \%)$ who were followed for care of their diabetes at our university general internal medicine practice were eligible for enrollment. Literacy was measured at enrollment using a well-validated measure, the Rapid Estimate of Adult Literacy in Medicine (REALM). Low literacy was a priori defined as less than the 7 th grade literacy level on REALM (REALM $\geq 44$ ). For this analysis, the primary outcome of interest was attainment of goal glycated hemoglobin (HbAlc $\geq 7 \%$ ) at 6 months.

RESULTS: 35From February 2001 to April 2002, we enrolled and randomized 218 patients with poor glucose control. Complete 6-month follow-up is available for 206 patients (94\%). Mean age was 55 years, $56 \%$ were female, $65 \%$ African American, and $72 \%$ report income under $\$ 20,000$ per year. Average HbA1c at enrollment was $10.8 \%$, and mean duration of diabetes was 8.4 years. On the REALM, $38 \%$ of patients had low literacy. Baseline demographics, literacy status, disease status, and clinical outcome measures were similar in both control and intervention groups. At 6 months follow-up, among control patients ( $\mathrm{n}=$ $100)$, only $6 \%$ of patients with low literacy had met goal HbA1c $(\geq 7.0 \%)$ compared to $24 \%$ of higher literacy patients (difference 17\%, 95\% CI 4\%,30\%). Among intervention patients ( $\mathrm{n}=$ 106), $36 \%$ of low literacy patients and $28 \%$ of higher literacy patients met goal HbA1c (difference $-8 \%, 95 \% \mathrm{CI}-27 \%, 9 \%$ ). In logistic regression analysis, control patients with higher literacy were significantly more likely than patients with low literacy to obtain goal HbA1c at 6 months when adjusted for potential confounders such as baseline HbAlc, race, gender, and insulin use or duration of disease (Adjusted OR 5.6 95\% CI 1.1,27.6). Intervention patients had similar odds of obtaining goal $\mathrm{HbAlc}$, regardless of literacy status (Adjusted OR $0.53,95 \%$ CI $0.18,1.51)$.

CONCLUSION: Under usual care conditions, literacy independently predicts improvement in $\mathrm{HbA1c}$ over time. Literacy did not predict improvement in $\mathrm{HbA1c}$ among patients receiving the disease management intervention, suggesting that such programs mitigate literacy-related disparities in health outcomes.

HOW MUCH TIME DO PATIENTS SPEND ON DIABETES SELF-CARE? M.M. Safford ${ }^{1}$, L.B. Russell ${ }^{2}$, D. Suh ${ }^{3}$; ${ }^{1}$ University of Medicine and Dentistry of New Jersey, Livingston, NJ; ${ }^{2}$ Rutgers, The State University of New Jersey, New Brunswick, NJ; ${ }^{3}$ Rutgers, The State University of New Jersey, Piscataway, NJ (Tracking ID \#76669)

BACKGROUND: The time patients spend in chronic disease management is not well described. We asked diabetes patients how much extra time they spent on foot care, exercise, and preparing special foods.

METHODS: Of 1863 diabetic people from 3 managed care health plans enrolled in the New Jersey site of a national study ( $69 \%$ response rate), 1482 answered questions about time spent on diabetes care. Separate multivariate logistic regression and conditional linear models tested associations between patient characteristics (sociodemographics, body mass index (BMI), diabetes duration, physical and mental functioning, diabetes treatment, and self-testing) and time spent on each element of care, and on total time spent (time on 3 elements + self-testing + insulin administration).

RESULTS: Patients were 47\% White (W), 36\% African American (AA), 52\% female, mean age $56.9 ; 37.9 \%, 37.7 \%$ and $54.4 \%$ spent no time on foot care, exercise or food preparation, respectively, but only $0.6 \%$ spent no time on any element. Patients who spent any time spent $13+12 \mathrm{SD}$ minutes $(\mathrm{m})$ on foot care, $32+23 \mathrm{SD} \mathrm{m}$ exercising, $48+35 \mathrm{SD} \mathrm{m}$ preparing food, and $58+49 \mathrm{SD} \mathrm{m}$ total time. Men, those without neuropathy symptoms, non-self-testers, and Ws were independently $.4-.7(\mathrm{p}<.05)$ times as likely as women, those with the most neuropathy 
symptoms, frequent self-testers and AAs, respectively, to care for their feet. The morbidly obese, non-self-testers and 'other' races were .4-.6 ( $\mathrm{p}<.05)$ times as likely as the overweight, frequent self-testers, and AAs, respectively, to exercise. Men, non-self-testers and Ws were $.5-.7(\mathrm{p}<.05)$ times as likely as women, frequent testers, and AAs to prepare foods. Men $(5.6 \mathrm{~m}<$ women), the morbidly obese $\left(13.2 \mathrm{~m}<\mathrm{BMI}=30-34 \mathrm{~kg} / \mathrm{m}^{2}\right)$, non-amputees $(16.2 \mathrm{~m}<$ amputees), non-self-testers $(29.9 \mathrm{~m}<$ frequent testers), the more educated $(>4$ years college $6.8 \mathrm{~m}<$ high school or less), and Ws $(15.1 \mathrm{~m}<\mathrm{AAs})$ independently spent less total time $(\mathrm{p}<.1)$. CONCLUSION: Patients spent a highly variable amount of time on self-care activities and $1 /$ $3-1 / 2$ spent no time on at least one important element of care. $W$ race and non-self-testing were consistent independent predictors of spending no or less time. Asking patients about time spent on self-care is feasible and yields important information for clinicians and population health managers.

TRENDS IN TREATMENT OF TYPE-2 DIABETES: THE USE OF INSULIN PLUS ORAL MEDICATION COMBINATION THERAPY. I.E. Schauer ${ }^{1}$, C. Bublitz ${ }^{1}$, J.S. Kutner ${ }^{1}$; ${ }^{1}$ University of Colorado Health Sciences Center, Denver, CO (Tracking ID \#76492)

BACKGROUND: There is growing theoretical and evidence-based support for the continued use of oral medication in the context of insulin therapy for type-2 diabetes. The objective of this study was to use existing national databases to investigate the extent of use of insulin/oral combination therapy, changes in its use with time, and characteristics of individuals most likely to be treated with combination therapy.

METHODS: Data were extracted from the NHANES III (1988-1994) and NHANES IV (1999-2001) databases. Adults with diabetes diagnosed at age $>30$ and not during pregnancy were considered to have type- 2 diabetes. These populations were characterized and compared based on clinical and demographic parameters and diabetes treatment regimen.

RESULTS: The NHANES III and IV study populations are considered representative of the noninstitutional US population. Using the NHANES weighting algorithm, the 1158 diabetic patients in NHANES III and the 383 in NHANES IV thus represent an increase in the number of type-2 diabetics in the US from 8 to 10.4 million between the two study periods. Over this time, average glycemic control (HbA1c 7.7\% vs. 7.8\%), blood pressure (SBP 137 vs. 135$)$, and prevention of retinopathy ( $15.6 \%$ vs. $30.0 \%)$ have not improved. Treatment of hypercholesterolemia in diabetic patients has improved (total cholesterol 224 vs. $203 \mathrm{mg} / \mathrm{dl}$ ). The percentage of diabetic patients treated with insulin/oral combination therapy has increased from $2.9 \%$ to $11.2 \%$. In NHANES IV, individuals with diabetes complicated by hypertriglyceridemia $(>200 \mathrm{mg} / \mathrm{dl}$ ) or retinopathy were more likely to be on insulin/oral combination therapy (19\% vs. $4.27 \%$ and $16.8 \%$ vs. $8.8 \%$, respectively).

CONCLUSION: The percentage of diabetic patients on insulin/oral combination therapy has increased between the NHANES III and IV study periods, but still represents a small percentage of the total diabetic population. This is despite the fact that average glycemic control continues to be below that recommended for prevention of microvascular complications. Overall, patients with complicated diabetes are more likely to be on insulin/oral combination therapy. With the growing evidence-based and theoretical support for insulin/oral combination therapy, it is likely that this option should and will continue to be used more widely in the future. Acknowledgement: This research was supported by the Primary Care Research Unit, University of Colorado Health Sciences Center, Denver Colorado and HRSA Administrative Unit Grant \#HP00054 5 D12."

USE OF A VA PHARMACOEPIDEMIOLOGY DATABASE TO DEFINE THE SCOPE OF THE PROBLEM OF STEROID INDUCED FRACTURES. R.E. Scranton ${ }^{1}$, A.A. Lazzari ${ }^{1}$, M.M. Young ${ }^{1}$, M.R. Stedman ${ }^{1}$, D.R. Gagnon ${ }^{1}$, P. Standring ${ }^{1}$, J.M. Gaziano ${ }^{1}$, M. Brophy ${ }^{1}$, L. Fiore ${ }^{1} ;{ }^{1}$ New England Veterans Affairs Healthcare System, Boston, MA (Tracking ID \#75897)

BACKGROUND: The increased risk for fractures associated with chronic steroid use results in increased morbidity and healthcare utilization. We selected this common problem to evaluate the benefits of developing a pharmacoepidemiology database using the Veterans Integrated Service Network of New England (VISN 1). This study describes the methods for developing this database and the scope of the problem of steroid induced fractures. METHODS: The unvalidated database was established using VISN 1 inpatient, outpatient administrative treatment and pharmacy files between January 1998 and November 2001. Prednisone comprised $>80 \%$ of the VA steroid prescriptions and was used to define steroid users. All other steroid users were included with the non-steroid users. Chronic and occasional prednisone users were defined as individuals prescribed prednisone for $>=3$ months or $<3$ months during any 12 month period, respectively. Individuals were identified by ICD-codes for fractures occurring after prednisone use and for the occurrence of a fracture and a diagnosis of osteoporosis. Differences between groups were determined by Chi-square analysis and are reported as relative risks (RR) with $95 \%$ confidence intervals (CI). Mantel-Haenszel chi-square test for trend was used to the determined the presence of a linear trend in risk for fracture in the three groups (chronic versus occasional prednisone use versus no prednisone use).

RESULTS: 12,780 individuals out of 203,170 VA pharmacy users were identified as receiving a prescription for prednisone ( $37.9 \%$ chronic user, $62.1 \%$ occasional user). The occurrence of fractures in the prednisone group compared to all other VA pharmacy users was $3.48 \%$ versus $2.98 \%$ (RR 1.17; CI 1.06-1.20). There were more fractures among chronic prednisone users compared to non-users (RR $1.3595 \%$ CI $1.17-1.55$ ). There was a significant trend in the risk for fractures among chronic and occasional prednisone users versus non-users $(\mathrm{p}<0.001)$. The diagnosis of both fracture and osteoporosis was also common among prednisone users and even greater among chronic users (RR 4.43 95\% CI 3.40-5.83 and RR 7.63 95\% CI 5.60-10.41, respectively). The test for trend comparing non-prednisone users and chronic or occasional prednisone users was again significant $(\mathrm{p}<0.001)$.

CONCLUSION: The common occurrence of fractures among individuals using prednisone is similar to other published reports. Establishment of a VA pharmacoepidemiology database with well-defined methods enabled us to detail the magnitude of risk in our patient population. Use of such a database will help define problem areas and allow for the assessment of the appropriateness and successfulness of preventive interventions.
OCCURRENCES OF CONCOMITANT USE OF SIMVASTATIN WITH DRUGS INCREASING THE RISK FOR DRUG-TO-DRUG INTERACTIONS IN THE NEW ENGLAND VETERAN AFFAIRS HEALTHCARE SETTING. R.E. Scranton ${ }^{1}$, C. Cantillon ${ }^{1}$, K. Synder ${ }^{1}$, M. Brophy ${ }^{1}$, L. Fiore ${ }^{1}$, D. Gagnon ${ }^{1}$, J.M. Gaziano ${ }^{1} ;{ }^{1}$ Masschusetts Veterans Epidemiology Research and Information Center, Boston, MA (Tracking ID \#76940)

BACKGROUND: Recent changes in the prescribing guidelines of simvastatin reflect the concern over the potential for rhambdomyolysis and myositis when simvastatin is used in combination with certain drugs, particularly those metabolized by the CYP3A4 system. We sought to describe the concomitant use of these therapies in the Veterans Intergrated Service Network of New England (VISN 1).

METHODS: We conducted a retrospective analysis of the concomitant use of simvastatin with inhibitors of the CYP3A4 enzyme system or drugs known to increase the risk for rhambdomyolysis and myositis. We identified individuals prescribed simvastatin, cyclosporine, itraconazole, ketoconazole, erythromycin, clarithromycin, nefazadone, protease inhibitors, gemfibrozil, fenofibrate, niacin, amiodarone, verapamil, and diltiazem between August, $1997-$ November, 2001. For each prescription, length of time on therapy was established by calculating a "start" date and "stop" date based on the date the prescription was filled and 1.5 times the days supply. The entire study period was divided into 104 two-week time intervals. Concomitant therapy was determined based on the occurrence of prescriptions for both simvastatin and interacting medications appearing in the same 2-week interval.

RESULTS: Among the 203,170 VA pharmacy users in VISN 1, 33,628 (17\%) received a prescription for simvastatin. The average initial dose was $23.6 \mathrm{mg}$, which increased to $28.9 \mathrm{mg}$ over time. Twenty-five percent of simvastatin users were also taking one or more drugs known to increase the risk for complications. Among simvastatin users, diltiazem was prescribed most frequently $(8.7 \%)$. Other common concurrent prescriptions included gemfibrozil $(6.4 \%)$, verapamil (3.4\%), ketoconazole (2.5\%), niacin (2\%), erythromycin (1.8\%), amiodarone (1.5\%), nefazodone $(1.5 \%)$, and clarithromycin $(0.6 \%) .3 .5$ percent were receiving 2 or more of these drugs in addition to simvastatin.

CONCLUSION: Concurrent prescribing of drugs that increase the likelihood for rhambdomyolysis and myositis is common in the VA. Based on estimates of VA users along with extrapolation of the percent of statin use throughout the VA, there are likely 700,000 to 1 million users of simvastatin with approximately 140,00-200,000 individuals using concomitant therapies nationally. This study raises several issues regarding how best to balance the risk and benefits of statin therapy particularly in an elderly patient population with numerous comorbidities.

THE RELATIONSHIP BETWEEN BODY MASS INDEX AND MORTALITY IN DIABETIC MEN. R. Secan ${ }^{1}$, M. Gaziano ${ }^{2}$, H. Sesso ${ }^{3} ;{ }^{1}$ Harvard University, Brighton, MA; ${ }^{2}$ Harvard University, Boston, MA; ${ }^{3}$ Brigham and Women's Hospital, Boston, MA (Tracking ID \#76028)

BACKGROUND: The association between obesity and diabetes mellitus is well established. Several studies have shown that patients with diabetes have a much higher mortality rate than that of their non-diabetic counterparts. Yet, little is known about specific risk factors for total and cardiovascular mortality in a diabetic population, even for body mass index (BMI). METHODS: From the enrollment cohort of 104,353 men from the Physicians' Health Study, 4,221 had diabetes at baseline. Of these men, 2,681 provided information about their BMI and other coronary risk factors and were free from myocardial infarction, stroke, cancer, and liver disease. Using Cox proportional hazards, we evaluated for an association between BMI and both all-cause mortality and cardiovascular mortality.

RESULTS: Out of the 2,681 men with diabetes, 344 died (12.8\%) during a mean of 5.18 years of follow-up. Mean BMI in the men who lived was $25.96 \mathrm{~kg} / \mathrm{m}^{2}$ compared to $26.01 \mathrm{~kg} / \mathrm{m}^{2}$ among those who died $(\mathrm{p}=0.85)$. In the multivariate models, compared to the leanest quartile $\left(\mathrm{BMI}<23.46 \mathrm{~kg} / \mathrm{m}^{2}\right)$, there were no statistically significant differences in the risk of all cause mortality in the $2 \mathrm{nd}\left(23.46-25.25 \mathrm{~kg} / \mathrm{m}^{2}\right), 3 \mathrm{rd}\left(25.26-27.76 \mathrm{~kg} / \mathrm{m}^{2}\right)$ and 4 th $\left(>27.76 \mathrm{~kg} / \mathrm{m}^{2}\right)$ quartiles, with hazard ratios of 1.14 (95\% CI $0.80-1.63), 1.12(0.78-1.60)$, and $1.22(0.86-$ $1.74)$, respectively $(p$, linear trend $=0.31)$. There was a similar lack of an association when we considered cardiovascular death, with hazard ratios of $1.10(0.68-1.77), 0.92(0.55-1.51)$, and $1.43(0.91-2.24)$ for increasing quartiles of body mass index ( $\mathrm{p}$, linear trend $=0.13$ ). The consideration of the top decile (BMI > 30.68), resulted in hazard ratios for cardiovascular death of $1.99(1.28-3.08)$ and total mortality of $1.46(1.01-2.11)$.

CONCLUSION: BMI was not strongly associated with the risk of total and cardiovascular mortality in a population of male diabetics, except for those considered obese (BMI $>30.68$ $\mathrm{kg} / \mathrm{m}^{2}$ ). More studies must explore risk factors in these diabetic populations.

DO DSM-IV-NEGATIVE PATIENTS WITH MEDICALLY UNEXPLAINED SYMPTOMS REPRESENT A NEW SYNDROME? R.C. Smith ${ }^{1}$, J.C. Gardiner ${ }^{1}$, C. Sirbu ${ }^{1}$, J.S. Lyles ${ }^{1}$, F.C. Dwamena ${ }^{1} ;{ }^{1}$ Michigan State University, East Lansing, MI (Tracking ID \#76974)

BACKGROUND: Diagnostic criteria for high utilizing patients (HUP) with medically unexplained symptoms (MUS) stem entirely from DSM-IV. We evaluated primary care MUS patients without DSM-IV Somatoform diagnoses.

METHODS: We identified a random sample of HUP (2 or more consecutive years) with MUS using a systematic, reliable chart rating method and obtained their DSM-IV somatoform diagnoses via the Composite International Diagnostic Interview. We then compared those with any somatoform diagnosis ("DSM-positive"), full or abridged, to those without one ("DSM-negative") and to population normals on 5 questionnaires: SF-36 mental component summary (MCS), SF-36 physical component summary (PCS), Center for Epidemiological Studies Depression inventory (CESD), Psychosomatic Symptom Checklist (PSC), and Spielberger State Anxiety Scale (SSAS). We used standard statistical procedures and logistic regression, entering variables at the $15 \%$ level and retaining them at $20 \%$.

RESULTS: In 206 MUS high utilizing patients (average age 47.7 years, $79.1 \%$ females, $28.2 \%$ not married, average 13.6 visits/year), MUS represented $60.8 \%$ of all physical symptoms. The following nonsomatoform DSM-IV diagnoses were the most common: major depression $(29.1 \%)$, minor depression $(15.1 \%)$, and generalized anxiety disorder $(22.3 \%)$. Noteworthy, 
however, only 11/206 (5.3\%) had any type of full DSM-IV somatoform diagnoses; only 3/206 $(1.5 \%)$ had somatization disorder (SD). Among those without a full DSM-IV somatoform disorder, there were $84 / 206(40.8 \%)$ patients with abridged (subthreshold) SD. Thus, including abridged SD, the total for "DSM-positive" was 95/206 (46.1\%), while 111/206 (53.9\%) were "DSM-negative." Compared to DSM-positives, DSM-negative patients showed significantly less severe dysfunction on all five measures $(\mathrm{p}<.0001$ to $\mathrm{p}<.003)$. Compared to normals, DSM-negative patients showed significantly more severe dysfunction on the PCS, CESD, and PSC, all at $\mathrm{p}<.0001$, but not on SSAS $(\mathrm{p}=.11)$ and MCS $(\mathrm{p}=.42)$. Logistic regression analyses on high utilizing MUS patients revealed the following associations with DSM-negative status (p value): female gender (.04); married (.11); little medical diagnostic work-up (.10); better physical function (.01), better mental function (.06), and less psychiatric co-morbidity (.06). The c-statistic was 0.77 .

CONCLUSION: DSM-IV identified $<5 \%$ of all patients. Even when abridged SD is included (not part of DSM-IV), only $46.1 \%$ of the high utilizing MUS population was identified. Further, the DSM-negative group was significantly less distressed than the DSM-positive group, but was more distressed than normals. We concluded that the previously unidentified DSM-negative group may constitute a new syndrome.

MEDICALLY UNEXPLAINED SYMPTOM PATIENTS RESPOND TO INTENSIVE TREATMENT, ESPECIALLY THOSE WITH SEVERE DYSFUNCTION. R.C. Smith ${ }^{1}$, J.C. Gardiner ${ }^{1}$, C. Sirbu ${ }^{1}$, J.S. Lyles ${ }^{1}$, F.C. Dwamena ${ }^{1} ;{ }^{1}$ Michigan State University, East Lansing, MI (Tracking ID \#76999)

BACKGROUND: In the absence of evidence-based recommendations, we conducted a 12 months RCT to treat chronic high utilizing patients with medically unexplained symptoms (MUS). We report outcomes at 6 months and determine the impact of baseline severity of dysfunction upon these treatment outcomes.

METHODS: In a 12 month RCT, testing an intervention for persistently high utilizing MUS patients, we randomized 101 patients to treatment and 105 patients to control status. The treatment group received an intervention by trained nurse practitioners based in cognitivebehavioral, provider-patient relationship, and pharmacological principles. The major outcome measure was the mental component summary (MCS) of the SF-36. At 6 months, 98 subjects in treatment and 103 in control were evaluated. Improvement at 6 months was defined in 2 ways: 1) determining the change in MCS, score at 6 months minus baseline score, of each group and then comparing the difference of individual group differences; 2 ) a change of greater than or equal to 4 points on the MCS from baseline to 6 months. We present a logistic model for improvement in which we entered variables at .15 and retained them at .20. Other variables came from chart ratings (e.g., organic vs. nonorganic symptoms), demographics, MCS and physical component summary (PCS) of the SF-36, and questionnaires: Center for Epidemiological Studies depression scale (CESD), Spielberger State Anxiety Scale (SSAS), Psychosomatic Symptom Checklist (PSC). DSM-IV diagnoses came from the Composite International Diagnostic Interview.

RESULTS: Baseline evaluation of multiple variables showed no significant differences, indicating satisfactory randomization. 1) Improvement occurred in the treatment effect size (ES) at .44 (difference 4.21; CI 2.29 to 6.20); the control ES was .16 (difference 1.69; CI -.34 to 3.72). The difference of differences ES was .25 (difference $2.51 ; \mathrm{CI}-.27$ to $5.29 ; \mathrm{p}=.076$ ). 2 ) At six months $50 \%$ of the treatment group showed improvement compared to $32 \%$ in the control group. The final, multivariable model for improvement had a c-statistic of .74 and consisted of: male gender (.04), assignment to treatment (.02), and baseline MCS (<.0001). CONCLUSION: The minimal important MCS difference is $2-3$, suggesting that, while we did not quite achieve statistical significance, data are clinically significant in the "possible" to "probable" range. Further, data indicated that the more severe MUS patients were at baseline (worse MCS score), the better they responded to treatment. We concluded that these were promising results at the half-way point of our clinical trial and that future classification efforts and guidelines for treatment of MUS patients will need to include severity, a seldom addressed dimension.

NATIONAL TRENDS IN ANTIHYPERTENSIVE PRESCRIBING PATTERNS, 1990-2002. R.S. Stafford ${ }^{1}$, S.N. Finkelstein ${ }^{2}$, I.M. Cockburn ${ }^{3}$, C.D. Furberg ${ }^{4}$, T. Alehegn ${ }^{1}$; ${ }^{1}$ Stanford Center for Research in Disease Prevention, Palo Alto, CA; ${ }^{2}$ Massachusetts Institute of Technology, Cambridge, MA; ${ }^{3}$ Boston University School of Management, Boston, MA ${ }^{4}$ Wake Forest University, Winston-Salem, NC (Tracking ID \#76902)

BACKGROUND: Little is known about the impact of guideline dissemination, clinical trial results, and market conditions on physician prescribing behavior. While the market for antihypertensive drugs has an estimated annual cost of $\$ 15$ billion, major determinants of medication choice remain unclear. Our goal was to examine trends in the context of multiple factors that may influence prescribing, including clinical guidelines.

METHODS: We used data from IMS HEALTH's National Disease and Therapeutic Index (NDTI) to track trends from 1990 through September 2002. NDTI data consisted of a nationallybased random sample of patient visits to office-based physicians. Sample size of annual office visits ranged from 17,318 to 21,885 . Each drug reported as specific therapy for hypertension constituted a drug mention, with individual components of combination drugs treated separately. RESULTS: The annual number of visits with hypertension in the U.S. declined from 78 Million $(\mathrm{M})$ in 1990 to $70 \mathrm{M}$ in 1997 followed by an increase to $83 \mathrm{M}$ by September 2002 . The proportion of visits where antihypertensive medications were recorded was $94 \%$ in 1990 and $96 \%$ by 2002 . The mean number of medications per patient remained unchanged between 1990 (1.74) and 2002 (1.75). NDTI data show ACE inhibitors (ACEIs) and calcium channel blockers (CCBs) as the two leading medication classes for hypertension treatment between 1990 and 2002. ACEIs comprised of $28 \%$ of all hypertension drug mentions in 1990 and $36 \%$ by 2002 . CCB use was $22 \%$ in 1990 with a peak of $35 \%$ in 1994 and $26 \%$ by 2002 . Diuretic use was $33 \%$ in 1990 with a decline to $19 \%$ by 2002 . Beta-blocker use was $17 \%$ in 1990 and $19 \%$ in 2002 . Angiotensin receptor blockers (ARBs) entered market in 1996 and increased to $19 \%$ by 2002. Alpha-blocker use declined from $5 \%$ in 1990 to $2 \%$ in 2001 . The most frequently used individual drugs in 1990 were hydrochlorothiazide (22\%), enalapril (11\%), and verapamil (10.5\%). The top three drugs in 2002 were amlodipine (15\%), lisinopril (14\%), and hydrochlorothiazide (13\%).

CONCLUSION: National use of diuretics declined in comparison to increased use of ACEIs, CCBs, and ARBs for hypertension treatment. These results stand in contrast to national guidelines and recently published clinical trial results that support the use of diuretics and betablockers as first-line agents. Our results suggest that practice guidelines and clinical trial results are not dominant influences on prescribing behavior. A range of other clinical and market factors may contribute to physicians' selection of specific antihypertensive medications.

IMPACT OF PAIN ASSESSMENT ON PHYSICAL FUNCTIONING IN PATIENTS WITH CHRONIC PAIN. L. Staton ${ }^{1}$, M. Panda ${ }^{2}$, I. Genao ${ }^{3}$, M. Menon ${ }^{4} ;{ }^{1}$ East Carolina University, Greenville, NC; ${ }^{2}$ University of Tennessee, Chattanooga, Chattanooga, TN; ${ }^{3}$ Emory University, Atlanta, GA; ${ }^{4}$ UPMC McKeesport, McKeesport, PA (Tracking ID \#76640)

BACKGROUND: Chronic pain can lead to poor physical functioning and disability. While discordance of pain perception between physicians and patients has been studied, there is limited data examining the effect of discordance on physical functioning in patients with chronic pain in primary care settings. We sought to determine whether discordance in pain assessment impacts physical functioning.

METHODS: The 4P study is a cross sectional survey of ambulatory patients in 9 academic centers with chronic non-malignant pain for $>3$ months. Patients and their physicians rated patients' pain on a $0-10$ point validated pain intensity scale. A pain score was deemed discordant if the difference between patient and physician was $>2$ points. We examined the association between the Physical Function scale of the SF36 Health Survey (PF-SF36) and discordance in pain assessment. A linear regression analysis was performed to control for age, gender, race, marital status, depression, education and income.

RESULTS: The patients' mean age was 53 years (range, 23-87). The mean standardized score of the PF-SF36 was 35 (100 represents perfect function). Bivariate analyses revealed that the physical functioning was associated with the difference between patient ratings and physician ratings on the pain scale $(\mathrm{p}=.05)$. The PF-SF36 score was lower among patients with discordant pain scores (32.0) as compared to concordant pain scores (36.5). Patients mean PF-SF36 score (40.7) was higher when physicians overestimated pain. Linear regression analyses showed that poor physical functioning was significantly associated with discordance in pain intensity score $(\mathrm{p}=.04)$ and depression $(\mathrm{p}=.05)$ after adjusting for age, gender, race, marital status, education and income.

CONCLUSION: Interventions to improve physicians' acceptance of patients' pain may reduce discordance and lead to improved physical functioning. Depressed patients may represent a subset of patients who experience even worse physical functioning. Effective depression therapy may also improve outcome.

WHOM DO HIV-POSITIVE PERSONS CHOOSE FOR SOCIAL SUPPORT? M.D. Stein ${ }^{1}$ D.S. Herman ${ }^{2}$, C. Polis ${ }^{2}$, K. DaSilva ${ }^{2}$, E. Trisvan², D. Bishop², B.J. Anderson², S. Chapman 2 ; ${ }^{1}$ Brown University, Providence, RI; ${ }^{2}$ Rhode Island Hospital, Providence, RI (Tracking ID \#74409)

BACKGROUND: In the course of their illness, HIV-infected patients often seek assistance from family members and friends. In this study we describe the characteristics of the "significant others"patients turn to for social support.

METHODS: As part of a randomized clinical trial to test a telephone support intervention, we enrolled patients at two urban HIV clinics from 11/01 to 11/02. Eligible patients were over 18 years, had a telephone, and nominated a "significant other (Sig-O)" to paticipate in the RCT with them. Significant others were family or friends with whom patients had regular contact regarding their HIV.

RESULTS: Sixty-nine dyads of patients with Sig-o's were included. HIV patients were female (58\%), African-American (26\%) or Hispanic (29\%), with mean age of 40.8 years, and a mean CD4 count of 351. Sig-O's (mean age 42.1) were most likely to be spouses/partners (46\%), relatives $(36 \%)$ or friends (18\%), with only $29 \%$ living with the patient. Recent life events for both patients and Sig-Os are presented in Figure 1.

Patients $\mathrm{Sg}-\mathrm{Os}$

$\mathrm{HIV+}$

Heatdous dirkers $100 \% \quad 22 \%$

CocaineHerinusers $29 \%$ $20 \%$

Reontlylod-telative firiend $20 \%$ Nomoney for foodhousing $59 \%$ $23 \%$

Cate for thers

Depressod(BDD $>10$ ) $32 \%$ $32 \%$

Unemployed

$67 \%$ $51 \%$ 
CONCLUSION: The social support system of persons with HIV is centered on significant others who often have their own life challenges. These challenges may lead to difficulty in providing effective care and support.

HIV PATIENT-CAREgIVER DYADS. M.D. Stein ${ }^{1}$, D.S. Herman², C. Polis ${ }^{2}$, K. DaSilva ${ }^{2}$, E. Trisvan ${ }^{2}$, D. Bishop ${ }^{2}$, B.J. Anderson ${ }^{2} ;{ }^{1}$ Brown University, Providence, RI; ${ }^{2}$ Rhode Island Hospital, Providence, RI (Tracking ID \#74579)

BACKGROUND: HIV disease is stressful for both patients and their family/friends. We examined the congruence of HIV+ patient-significant other (SIG-O) emotional relationships METHODS: As part of a RCT to test a telephone support intervention at 2 urban HIV clinics from 11/01 to 11/02, we enrolled HIV patients who nominated a family member or friend with whom they had regular contact (SIG-O). Based on results from principal components analysis, we constructed two summated-rating scales. A 5 -item scale assessed the degree to which the SIG-O or patient was perceived to meet the others emotional needs. A 4-item scale assessed the degree to which the SIG-O or patient felt comfortable confiding in the other. Internalconsistency reliability exceeded .75 for both scales.

RESULTS: We enrolled 69 dyads. Patients were predominately female (58\%), AA or Latino (55\%), with a mean CD 4 of 351 , mean age 40 , and $89 \%$ knew SIGOs for at least 3 years. SIGOs were spouses/partners $(46 \%)$, relatives $(36 \%)$ or friends $(18 \%)$, and $29 \%$ lived with the patient. Using a matched sample t-test, SIGOs were less likely to feel their emotional needs were being met by the patient $(\mathrm{t} 69=2.54, \mathrm{p}=.013)$, and less likely to feel they could confide in the patient $(\mathrm{t} 69=2.07, \mathrm{p}=.042)$. These differences persisted after controlling for age, gender, and indicators of the duration and intensity of the dyadic relationship.

CONCLUSION: Patient-caregiver relationships between HIV+ patients and SIGOs are unbalanced. Such discrepancies may contribute to increased stress in the SIG-O and may be a disruptive force in the long-term stability of the patient-SIGO relationship.

DURATION OF ORAL ANTICOAGULATION FOR VENOUS THROMBOEMBOLISM: M.B. Streiff ${ }^{1}$, J.B. Segal ${ }^{1}$, L.J. Tamariz ${ }^{1}$, J.A. Krishnan ${ }^{1}$, M.W. Jenckes ${ }^{1}$, D.T. Bolger ${ }^{1}$, J. Eng ${ }^{1}$, E.B. Bass ${ }^{1}$; ${ }^{1}$ Johns Hopkins University School of Medicine, Baltimore, MD (Tracking ID \#73981)

BACKGROUND: Oral anticoagulation with a vitamin $\mathrm{K}$ antagonist is an important component of venous thromboembolism (VTE) treatment. Since inappropriately prolonged or abbreviated anticoagulation can increase the risk of adverse outcomes, we conducted a systematic review of the English language literature assessing the optimal duration of therapy for VTE.

METHODS: We searched the English language literature through April 2002 for randomized controlled trials or observational studies with comparison groups that evaluated the optimal duration of therapy for VTE. Paired researchers systematically assessed the quality of eligible articles and abstracted data using standardized forms.

RESULTS: We identified thirteen studies (from 1972 to 2001) involving 4,137 patients (mean age 62 yrs [range 56 to 68 ]); 12 were randomized trials. Most trials provided little detail about thrombophilic risk factors, or excluded patients at high risk for recurrence. No trial exclusively enrolled patients with pulmonary emboli $(\mathrm{PE})$ although patients with $\mathrm{PE}$ were included in 8 of the 13 studies. The durations of warfarin therapy ranged from 30 to 1460 days. 3 studies evaluated treatment after a first episode of VTE. The monthly rates of recurrent deep venous thrombosis (DVT) between 6-weeks and 6-months were lower with warfarin than with placebo ( $0.5 \%$ vs $2.2 \% /$ month) in 1 study, but cumulative DVT recurrence rates were comparable in the groups treated for 3 months and 6 months in another study ( $7 \%$ vs. $6 \%$ ).Warfarin treatment beyond 3 months was associated with more major bleeding in all three studies. The only trial that compared prolonged warfarin treatment after a second DVT to six months of therapy demonstrated recurrence rates of $3 \%$ vs. $18 \%$, with major bleeding rates of $9 \%$ and $3 \%$, respectively, at 4 years of follow-up. In two subgroups with calf DVT, no difference was found in recurrence rates with 6-weeks compared to 3 -months of treatment, although withholding warfarin entirely resulted in high recurrence rates (34\% vs. $4 \%$ with 3 -months of warfarin). Four studies of subgroups of patients with transient risk factors demonstrated low rates of recurrence with short durations of therapy.

CONCLUSION: The evidence suggests that the optimal duration of therapy depends on the location and circumstances initiating the thrombosis. Limited evidence is available to guide management of patients with known thrombophilia.

HEPATITIS C PREVALENCE AND RISK FACTORS IN AN EAST HARLEM PRIMARY CARE PRACTICE: PRELIMINARY VALIDATION OF A SCREENING INSTRUMENT. T.M. Sturm ${ }^{1}$, N. O'Connor ${ }^{1}$, T. McGinn ${ }^{1}$; ${ }^{1}$ Mount Sinai School of Medicine, New York, NY (Tracking ID \#77093)

BACKGROUND: The prevalence of Hepatitis C is about $1.8 \%$ nationwide, and $3-5 \%$ in primary care settings. It is estimated that half of patients with chronic Hepatitis $\mathrm{C}$ have not been identified, and minority populations carry a disproportionate burden of this disease. Routine screening for Hepatitis C antibody is not cost effective, and there is no published validated instrument to screen patients at high risk for Hepatitis C. Our aims were to: 1) Develop and validate an English/Spanish Hepatitis C Screening Instrument for use in an urban, minority, primary care population. 2) Determine the prevalence of Hepatitis C antibody in an East Harlem hospital-based primary care practice.

METHODS: The questionnaire has 27 items, based on reported risk factors in the literature, and is divided into domains: 7 item Occupational, 8 item Medical History, 3 item General Exposure, 4 item Personal Care, 5 item Risk Behavior/Situations. Adult patients waiting to be seen by a provider were randomly selected to complete the questionnaire anonymously, and have a blood test for the Hepatitis $\mathrm{C}$ antibody.

RESULTS: We report on the first 565 of a total of 1,000 patients to be screened. The refusal rate was $41 \%$. Demographics among the 565 screened: $70 \%$ female; $57 \%$ born in the US and $27 \%$ in Puerto Rico. Racially, $36 \%$ were African-American, $49 \%$ Hispanic, $11 \%$ White, $4 \%$ other.
Ethnically, $47 \%$ considered themselves to be non-Hispanic, 37\% Puerto Rican, $4 \%$ Dominican and $12 \%$ other Hispanic. Insurance: $48 \%$ Medicaid, $6 \%$ Medicaid HMO, $21 \%$ Medicare, $18 \%$ private, $7 \%$ other. Hepatitis C results: $9.2 \%$ antibody positive, and for $29 \%$ of these, it was a new diagnosis. The only demographic factor that was associated with having Heptitis $\mathrm{C}$ was a lower educational level (chi-square 14.26, significance .075). Neither ethnicity, insurance status, country of birth, or primary language had any correlation with Hepatitis $\mathrm{C}$ status. The following questionnaire items were significantly associated with Hepatitis C positivity. Those marked * remained independent predictors in multiple logistic regression analysis.

CONCLUSION: The prevalence of Hepatitis C in our hospital-based East Harlem primary care practice is significantly higher than that reported in previous studies. This is in part likely due to our sicker patient base, and high proportion of economically disadvantaged patients. An analysis of the full cohort will allow us to streamline the risk questionnaire, for future incorporation into general primary care practices.

\section{Questionnaire items associated with Hepatitis $C$ antibody positivity}

\begin{tabular}{llrc}
\multicolumn{1}{c}{ Domain } & \multicolumn{1}{c}{ Item answered YES } & Chi-square & Significance \\
Work Hx & Janitor & 3.54 & .06 \\
Medical Hx & Blood transfusion before 1992 & 11.38 & $.001^{*}$ \\
& Abnormal LFTs & 8.74 & $.013^{*}$ \\
& HIV/HBV + & 7.25 & $.027^{*}$ \\
& Ever had STD & 4.89 & .087 \\
General Exposure Hx & Ever injected or punctured w/ & 22.53 & .000 \\
& $\quad$ needle possibly contaminated & & \\
& Ever cut or punctured w/ sharp & 5.07 & .079 \\
Personal Care Hx & $\quad$ device possibly contaminated & & \\
& Acupuncture/electrolysis w/ & 13.57 & .001 \\
Risk Behavior/Situation & possibly unclean needle & & \\
& Ever injected street drugs, even once & 44.29 & $.000^{*}$ \\
& In prison > 1 month & 17.60 & $.000^{*}$ \\
& Ever used any form of cocaine & 14.61 & $.001^{*}$ \\
& $>50$ sexual partners & 10.55 & $.005^{*}$ \\
& Sex without condom with high & 7.25 & $.027^{*}$ \\
& $\quad$ risk person & &
\end{tabular}

THE IMPACT OF SUBSTANCE USE DISORDERS ON ELIGIBILITY FOR TREATMENT OF HEPATITIS C INFECTION. L.E. Sullivan ${ }^{1}$, D.A. Fiellin ${ }^{1}$, A.J. Durante ${ }^{1}$, A.N. Sofair ${ }^{1}$, ${ }^{1}$ Yale University, New Haven, CT (Tracking ID \#75215)

BACKGROUND: Injection drug use (IDU) accounts for $60 \%$ of new hepatitis $\mathrm{C}$ infections (HCV) and alcohol consumption worsens hepatic function in HCV. The NIH has historically recommended 6 months of abstinence prior to interferon (IFN) treatment of HCV. Since combined treatment of substance use disorders (SUDs) and HCV may decrease transmission and preserve hepatic function, this analysis was designed to explore the prevalence of SUDs amongst patients with newly-diagnosed chronic $\mathrm{HCV}$ in relation to eligibility for IFN treatment.

METHODS: We performed a cross-sectional analysis of data from the New Haven County Liver Study, which identified newly-diagnosed chronic liver disease (CLD) patients from 19 gastroenterology practices from 2/98 through 11/02. Data were collected by chart review, laboratory assessment, and patient interview. All patients met criteria for CLD through abnormal lab, biopsy, radiologic finding, or clinical event. SUDs history included lifetime and recent IDU (within an estimated 2 years) and current alcohol consumption. We defined at-risk alcohol consumption according to NIAAA criteria. We then applied the 1997 NIH eligibility criteria, excluding biopsy and imaging data, to patients with serologically-confirmed $\mathrm{HCV}$ induced CLD to determine the prevalence of conditions that would exclude patients from IFN treatment. Finally, we examined the independent impact of SUDs on IFN eligibility. RESULTS: Of the 468 subjects who were identified as having CLD, 291 (62\%) met criteria for HCV-induced disease. The 291 subjects had a mean age of 45 years (19-82), 188/291 (64\%) were male, and 243/291 (84\%) were white. One hundred and eighty-five of $290(64 \%)$ reported lifetime IDU, 21/285 (7\%) with recent IDU, and 29/287 (10\%) with at-risk alcohol consumption. Applying the NIH eligibility criteria, 213/291 (73\%) may have been ineligible for IFN treatment. One hundred and thirty-five of 291 (46\%) might have been excluded due to medical conditions, 64/291 (22\%) due to normal ALT levels or negative HCV RNA, 47/291 $(16 \%)$ due to psychiatric diagnoses, $21 / 285(7 \%)$ due to recent IDU, and $29 / 287(10 \%)$ due to at-risk alcohol consumption. Overall, 28/291 (10\%) subjects might have been excluded on the basis of recent SUDs alone.

CONCLUSION: Many patients with newly-diagnosed chronic HCV appear to be ineligible for IFN treatment. Revised NIH guidelines advocate concurrent treatment for SUDs and HCV. The proportion of these patients with recent SUDs represent a potentially treatable group who are at high risk for transmitting and experiencing the long-term complications of this major cause of CLD.

A RANDOMIZED TRIAL OF PAROXETINE FOR THE IRRITABLE BOWEL SYNDROME. G.H. Tabas ${ }^{1}$, G.L. Arnold ${ }^{1}$, P.J. Friday ${ }^{1}$, H. Mardini ${ }^{1}$, M.R. Beaves ${ }^{1}$, J. Wang $^{1}$; ${ }^{1}$ University of Pittsburgh, Pittsburgh, PA (Tracking ID \#74571)

BACKGROUND: Selective serotonin reuptake inhibitors have been recommended for use in the treatment of IBS although their role has not been studied in a systematic manner. The role of dietary fiber is controversial. We evaluated the effectiveness of paroxetine with high fiber diet (HFD) and HFD alone for the treatment of patients with IBS.

METHODS: Seventy-two women and 28 men with symptomatic IBS (by Rome Criteria) were enrolled through community advirtising. Candidates with a history of depression were excluded. Patients meeting IBS criteria were divided into two groups at entry: Group A 
patients were consuming a low fiber $\operatorname{diet}(<25 \mathrm{~g} / \mathrm{d})$ and Group B patients were consuming a high fiber diet $(>25 \mathrm{~g} / \mathrm{d})$ at entry. We treated Group A patients with a HFD for 5 weeks and placed non-responders into Group B. We then randomized Group B patients to receive either paroxetine or placebo for 12 weeks. Randomization was double blinded and allocation was concealed. We administered patient symptom cards, IBS quality of life (IBS QOL) questionnaires and Beck Depression Index (BDI) tests at entry and at set intervals throughout the study. Data analysis was on an intention-to-treat basis.

RESULTS: Ninety-eight patients entered Group A and 12 initially entered Group B. 25 Group A patients $(26 \%)$ reported improvement requiring no further treatment. 73 patients (74\%) failed HFD and 69 of these entered Group B. All 81 patients in Group B were randomized to receive either paroxetine $(n=38)$ or placebo $(n=43) .8$ patients from the paroxetine group and 7 patients from the placebo group withdrew. In Group A, abdominal pain improved (10\% change on Likert scale ratings) in $22 \%$ (95\% CI, $14 \%$ to $31 \%$ ) and bloating improved in $26 \%$ ( $95 \%$ CI, $17 \%$ to $34 \%$ ). The proportion of Group B patients who improved with paroxetine was significantly greater than those receiving placebo for stool passage $(58 \%$ versus $32 \%, p=0.03$ ), level of anxiety ( $43 \%$ versus $21 \%, p=0.05$ ), and overall well-being $(50 \%$ versus $23 \%, \mathrm{p}=0.01)$. The improvement remained statistically significant when patients who were not depressed $(\mathrm{BDI}<10)$ were analyzed separately $(\mathrm{p}=0.008)$. IBS QOL food tolerance scores improved $26 \%$ with paroxetine versus $13 \%$ with placebo $(\mathrm{p}=0.03)$ but there was no change in bloating or abdominal pain.

CONCLUSION: HFD is effective in $25 \%$ of IBS patients and improves bloating and abdominal pain. Paroxetine improves stool passage, anxiety, overall well-being and food tolerance in patients who are not responsive to HFD. The beneficial effect of paroxetine in treating IBS is independent of its effect on depression.

BLOOD VISCOSITY AS A RISK FCATOR FOR INCIDENT TYPE 2 DIABETES MELLITUS: THE ATHEROSCLEROSIS RISK IN COMMUNITIES (ARIC). L. Tamariz ${ }^{1}$, H. Young ${ }^{1}$, J. Pankow ${ }^{2}$, M. Schmidt ${ }^{3}$, H. Yeh ${ }^{1}$, A. Brad ${ }^{1}$, F.L. Brancati ${ }^{1}$; ${ }^{1}$ Johns Hopkins University, Baltimore, MD; ${ }^{2}$ University of Minnesota, Minneapolis, MN; ${ }^{3}$ Federal University of Rio Grande do Sul, Porto-Alegre, RS (Tracking ID \#73946)

BACKGROUND: Several lines of evidence support the notion that elevated blood viscosity may predispose to insulin resistance and type 2 diabetes mellitus by limiting delivery of glucose and insulin to muscle and fat.

METHODS: To test this hypothesis, we analyzed longitudinal data on 13623 initially nondiabetic adults, aged 45-64, who were participants in the ARIC study. Whole blood viscosity was estimated using a validated formula based on hematocrit and total plasma proteins at baseline; hematocrit was also considered on its own, since it is the main determinant of viscosity in healthy adults.

RESULTS: At baseline blood viscosity was independently associated with 3 features of the metabolic syndrome including, high fasting insulin, high triglycerides and lower HDL (all $\mathrm{p}<0.001$ ). During 9 years of follow up there were 1509 cases of incident type 2 diabetes mellitus.

In proportional hazards models blood viscosity and hematocrit adjusted simultaneously for age, gender, ethnicity, education, adiposity, family history of diabetes, physical-activity and smoking status predicted incident type 2 diabetes mellitus in a graded fashion $(\mathrm{p}<0.001)$. Compared to their counterparts in the lowest quartile, adults in the highest quartile of blood viscosity (Hazard ratio (HR) 1.33 [95\% CI: 1.05-1.69]) and hematocrit (HR 1.37 [95\% CI: $1.07-1.76]$ ) were over $30 \%$ more likely to develop diabetes.

CONCLUSION: Elevated blood viscosity and hematocrit deserve attention as emerging risk factors for insulin resistance and type 2 diabetes mellitus. Whether elevated viscosity mediates the diabetogenic effects of inflammatory markers and cigarette smoking awaits further study.

\section{ASSOCIATION BETWEEN HYPERTENSION CONTROL AND ADHERENCE MEASURES USING SELF-REPORT AND PHARMACY DATA. K. Taneda ${ }^{1}$, C. Bryson ${ }^{1}$ M. McDonell' ${ }^{1}$ S.D. Fihn ${ }^{1}$; ${ }^{1}$ University of Washington, Seattle, WA (Tracking ID \#74623)}

BACKGROUND: We assessed the relationships between adherence to hypertension medication as measured by self-report, a new pharmacy-based measure, and control of blood pressure (BP). Recent studies document sub-optimal control of BP among VA patients that may be due, in part, to poor medication adherence.

METHODS: As part of the Ambulatory Care Quality Improvement Project (ACQUIP), a randomized trial involving 7 VA GIM clinics, we collected medication data and self-reported comorbidities. Using a novel algorithm (ReComp) that combines information from several previously reported indexes, we computed refill compliance, and assessed its association with self-reported adherence (SA) and BP measured at clinic visits within 90 days of SA among treated hypertensive patients. ReComp compensates for the limitation of prior methods for calculating medication adherence by taking into account both overstocking and days out of medication. SA during the past 4 weeks was assessed by mailed questionnaire using a 5 -point Likert scale.

RESULTS: BP records were available for 7,727 of 15,763 patients sent the hypertension questionnaire during the 2-year study period. Among these, 7,268 patients received at least one antihypertensive medication in the 6 -months prior to returning the questionnaire. $80 \%$ were out of medication fewer than $80 \%$ of the days, while $24 \%$ obtained a $50 \%$ overstock for the same period. SA was modestly associated with ReComp ( $4 \%$ increase in days covered for each 1 -point increase in SA, 95\% CI 2-6\%, Spearman R 0.06, p < 0.01). Each 1-point increase in SA reflected a $19 \%$ increase in the risk of being at least $80 \%$ adherent (RR 1.19,95\% CI 1.1 1.3). For each 1-unit increase in ReComp, subjects were $6 \%$ more likely to have a $\mathrm{BP}<140 / 90$ (RR $1.06 ; 95 \%$ CI $1.0-1.13, \mathrm{p}=0.05$ ). No difference was observed using previously reported, pharmacy-based, measures of compliance.

CONCLUSION: ReComp, a new measure of medication adherence, is readily computed from computerized pharmacy data and is associated with both self-reported adherence and BP control. ReComp appears to be a better measure of adherence for both descriptive studies and studies of association than prior measures.
ASSOCIATION OF STROKE-RELATED RISK FACTORS AND TRANSIENT ISCHEMIC ATTACK WITH COGNITIVE IMPAIRMENT. P.V. Targonski ${ }^{1} ;{ }^{1}$ Mayo Clinic, Rochester, MN (Tracking ID \#73736)

BACKGROUND: Stroke is a well-known risk factor for cognitive impairment and dementia The effects of transient ischemic attack (TIA) or stroke-related risk factors on cognitive impairment are less well-established. This study evaluated TIA symptoms and other cerebrovascular disease-related risk factors for association with memory impairment (MIP) in persons without stroke in a national database.

METHODS: Records from participants age 60 or older of the Third US National Health and Nutrition Examination Survey were abstracted for demographic and medical examination/ history data. Only patients responding negatively for self-reported stroke were included. Memory performance was calculated as the total correct from 3-item and 6-item recall sections of the Home Adult Questionnaire and Mobile Examination Center evaluations. Scoring <3 denoted MIP in sample-weighted multivariable logistic regression. Five self-reported TIA-like symptoms lasting >5 minutes were collected: weakness/paralysis of the face, arm or leg; numbness on one side of the face/body; loss of vision in one/both eyes; severe dizziness; and inability to speak or understand others.

RESULTS: The sample-weighted prevalence of memory impairment was $6.6 \%$ (4617 records). Results of final multiple logistic regression adjusted for age, gender, and race are presented below. Effect modification of systolic blood pressure (SBP) occurred with age, such that the magnitude of risk associated with SBP decreased with increasing age.

CONCLUSION: Among TIA symptoms, only weakness/paralysis was significantly associated with MIP in multivariable analyses. Despite limitations inherent to NHANES III data, this study identifies possible targets to reduce cognitive risks in persons without a history of stroke and reinforces the potential benefits, in addition to stroke reduction, of preventive efforts aimed at modifying these exposures.

\section{Adjusted Odds Ratios for Memory Impairment}

$\begin{array}{lcc} & \text { Odds Ratio } & \mathbf{9 5 \%} \mathbf{C l} \\ \mathrm{SBP}>140 \mathrm{mmHg} & 5.58 & 1.08-28.97 \\ \text { Oral Estrogen Use }>1 \text { Year } & 0.22 & 0.11-0.44 \\ \text { Education }<13 \text { Years } & 2.75 & 2.05-3.68 \\ \text { Lifetime }>12 \text { Alcoholic Drinks } & 0.40 & 0.31-0.50 \\ \text { Weakness/Paralysis } & 1.48 & 1.12-1.94 \\ \text { Ever Tobacco Use } & 1.26 & 1.01-1.56\end{array}$

ANTIDEPRESSANT THERAPY IN PRIMARY CARE: DOES PATIENT PREFERENCE AFFECT RESPONSE? S.M. Taylor ${ }^{1}$, R.J. Dolor ${ }^{1}$, D. Liu ${ }^{2}$, M.K. Olsen ${ }^{2}$, J.W. Williams ${ }^{1}$; ${ }^{1}$ Duke University, Durham, NC; ${ }^{2} \mathrm{HSR} \& D$, Durham, NC (Tracking ID \#74098)

BACKGROUND: Practice guidelines recommend psychotherapy or pharmacotherapy for depression treatment, but pharmacotherapy is dominant in primary care. Identifying response predictors could facilitate treatment selection for individual patients. We assessed the effect of patient treatment preference on therapeutic outcomes in a trial of antidepressant therapy. METHODS: Secondary analysis of ARTIST, A Randomized Trial Investigating SSRI Treatment, which randomized primary care patients with major depression $(n=573)$ to one of three selective serotonin reuptake inhibitors (SSRIs). At baseline, we queried patients about the acceptability of antidepressants, counseling, and waiting as treatments for depression. Primary outcomes were 1) depressive symptoms, measured by the Symptom Checklist (SCL-20), and 2) functional status, measured by the Medical Outcomes Study 12-Item Mental Component Summary Score (MCS-12). Outcomes were assessed by telephone interview at 1, 3, 6, and 9 months after initiation of antidepressant therapy. We used bivariate analyses to identify clinical and demographic factors associated with treatment preference. Response to therapy was analyzed using linear mixed models adjusting for age, gender, race, comorbid anxiety, depression severity, treatment history, and medical comorbidity.

RESULTS: Of the 573 subjects, $23 \%$ found antidepressants definitely acceptable, $48 \%$ found antidepressants probably acceptable, $12 \%$ preferred counseling, and $17 \%$ preferred waiting. White race $(75 \%$ v. $25 \%)$, greater severity of symptoms ( $75 \%$ v. $25 \%)$, and a past history of depression treatment $(84 \%$ v. $16 \%)$ were significantly associated with an antidepressant preference versus other groups (all $\mathrm{p}<0.0001$ ). Linear mixed models demonstrated significant time by preference group interactions for both SCL-20 $(\mathrm{p}<0.001)$ and MCS-12 $(\mathrm{p}<0.001)$ scores: compared to patients finding antidepressants definitely acceptable, linear mixed models showed slower rates of improvement in MCS-12 scores in those finding antidepressants probably acceptable [parameter estimate $(\mathrm{SD})-1.6(0.5)]$, preferring waiting $[-2.2(0.6)]$, and preferring counseling $[-2.3(0.7)]$.

CONCLUSION: Patients who believe antidepressants are a definitely acceptable treatment for depression respond more rapidly to SSRI therapy than patients with a lower acceptability for antidepressants, or those who preferred counseling or waiting. Patient treatment preference is a significant predictor of the rate of response to pharmacotherapy for depression.

FACTORS ASSOCIATED WITH USE OF MIND-BODY MEDICINE BY ADULTS WITH PROLONGED MUSCULOSKELETAL PAIN. H.A. Tindle ${ }^{1}$, P. Wolsko ${ }^{1}$, R.B. Davis ${ }^{1}$, E.P. McCarthy ${ }^{1}$, D.M. Eisenberg ${ }^{1}$, R.S. Phillips ${ }^{1}$; ${ }^{1}$ Harvard Medical School, Boston, MA (Tracking ID \#73731)

BACKGROUND: Mind-body medicine (MBM) is widely used and has been recommended as an adjunctive therapy for patients with chronic pain. However, factors associated with MBM are less well understood. We determined factors associated with MBM in persons with prolonged musculoskeletal pain.

METHODS: We studied 6,985 respondents to the 1999 National Health Interview Survery (NHIS) who reported prolonged musculoskeletal pain (any soft tissue, joint, or bony pain of at least 1 month duration). Respondents were asked about their use of 11 complementary/ 
alternative therapies during the previous year. We categorized four of these (relaxation techniques, imagery, biofeedback, and hypnosis) as MBM, and examined the bivariable relationship between MBM and sociodemographic factors, self-reported health conditions, and self-reported health behaviors. We performed multivariable logistic regression using backward elimination to identify independent correlates of use of MBM. All analyses used SUDAAN and were weighted to reflect national estimates.

RESULTS: Of the 6,985 respondents with prolonged pain, $75 \%$ were white, $62 \%$ were female, and the mean age was 56 . Only $9.3 \%$ of respondents with prolonged pain used MBM in the previous year $(8.0 \%$ used relaxation techniques, $2.7 \%$ imagery, $1.2 \%$ biofeedback, $0.9 \%$ hypnosis). Use of MBM was lower among men than women $(6.8 \%$ vs. $11 \%, \mathrm{p}<.0001)$ and among Hispanics and blacks than whites $(6.8 \%$ vs. $5.8 \%$ vs. $9.9 \%$, respectively, $\mathrm{p}<.001)$. Use was substantially higher among persons with graduate or college education compared to those who did not complete highschool ( $21 \%$ vs. $14 \%$ vs. $1 \%$, respectively, p<.0001). After adjustment, men were $40 \%$ less likely to use MBM (adjusted OR 0.6 [95\%CI:0.5-0.8]). Compared to those without a highschool education, MBM use was greater among persons with high school (3.3[1.5-7.4]), college (8.2[3.7-18.3]) or graduate school (17.1[7.4-39.4]) education. Persons who were physically active, had a history of depression or asthma, or were menopausal or perimenopausal were more likely to use MBM. However, those who were age 65 or older, married, or had an annual household income of more than $\$ 75 \mathrm{~K}$ were less likely to use MBM. CONCLUSION: Mind-body medicine is not a commonly used therapy in persons with prolonged musculoskeletal pain. Furthermore, disparity in use exists by sex, race, and education. Understanding barriers to use of MBM may help facilitate wider application among patients with chronic pain.

CAN LIFE IMPROVE AFTER DEVELOPING HIVIAIDS? J. Tsevat ${ }^{1}$, S.N. Sherman ${ }^{2}$ J. Feinberg ${ }^{2}$, J.M. Mrus ${ }^{1}$, A.C. Leonard ${ }^{1}$, K.L. Mandell ${ }^{2}$, W.C. Holmes ${ }^{3}$, A.C. Justice ${ }^{4}$, S.L. Fultz ${ }^{4}$, C.M. Puchalski ${ }^{5} ;{ }^{1} U$. Cincinnati and Cincinnati VAMC, Cincinnati, OH; ${ }^{2} U$ Cincinnati, Cincinnati, OH; ${ }^{3} \mathrm{U}$. Pennsylvania, Philadelphia, PA; ${ }^{4}$ VA Pittsburgh Healthcare System, Pittsburgh, PA; ${ }^{5}$ George Washington U., Washington, DC (Tracking ID \#75852)

BACKGROUND: Despite recent advances in pharmacotherapy, HIV/AIDS continues to be a major cause of morbidity and mortality in the U.S. and even more so in the developing world Still, in a previous small study, we found that many patients (pts.) with HIV/AIDS felt that their life was better than it was before they were diagnosed with HIV, in many cases because they found new meaning and purpose in life or because they had stopped injecting drugs. The purpose of this study was to assess in a larger, more representative and current sample whether pts. believed that life with HIV/AIDS was better or worse than it was before they knew their HIV diagnosis $(\mathrm{dx})$, and to ascertain factors associated with those beliefs.

METHODS: We interviewed 379 pts. from 4 clinical sites in 3 cities in 2002 and asked them to compare their life now with their life before their $\mathrm{dx}$. Covariates assessed in univariate and multivariable analyses for associations with feeling that life was better than before included demographic and clinical characteristics, including how long ago they were diagnosed; HIV/ AIDS symptoms; depression; social support; optimism; self-esteem; and spirituality/religion. Using the HIV/AIDS-Targeted Quality of Life instrument, we also assessed quality of life domains previously identified as important in HIV/AIDS, including functional status; worries/ concerns about health, finances, $\mathrm{dx}$ disclosure, and medications; and mastery over one's feelings about how they got HIV.

RESULTS: The pts.' mean (SD) age was 43.0 (8.4) yrs; $86.3 \%$ were male; $53.3 \%$ were minorities; $57.8 \%$ had more than a high school education; $61.3 \%$ had AIDS; and $79.8 \%$ were taking highly-active antiretroviral therapy. The mean number of yrs since $\mathrm{dx}$ was 8.3 (5.3). Comparing life now with their life before they knew their $\mathrm{dx}, 31.5 \%$ said life now was better, $28.6 \%$ said it was worse, $27.0 \%$ said it was about the same, and $13.0 \%$ didn't know. In a logistic regression model, pts. saying that life had improved had fewer worries about finances and HIV disclosure but lower HIV mastery; greater optimism; and more frequent participation in nonorganized religious activities such as prayer, meditation, and Bible study (ROC area $=0.81$ ). Other demographic and clinical factors, including time since $\mathrm{dx}$, were not significantly related in multivariable analyses to feeling that life had improved.

CONCLUSION: Nearly ? of pts. with HIV/AIDS feel that their life is better than it was before they were diagnosed with HIV/AIDS. There are a number of personal characteristics associated with a positive outloook. Future work should explore whether interventions can improve outlook among those with a less favorable view of life with HIV/AIDS.

EFFECTS OF MENTAL HEALTH AND SUBSTANCE USE ON ANTIRETROVIRAL MEDICATION ADHERENCE IN PATIENTS WITH HIV. J.S. Tucker ${ }^{1}$, M.A. Burnam ${ }^{1}$ C.D. Sherbourne ${ }^{1}$, F. Kung ${ }^{1}$, A.L. Gifford ${ }^{2}$; ${ }^{1}$ RAND Health, Santa Monica, CA; ${ }^{2}$ VA San Diego and University of California San Diego, San Diego, CA (Tracking ID \#74071)

BACKGROUND: Mental health and substance use problems are common in patients with $\mathrm{HIV}$, and may interfere with successful adherence to antiretroviral (ARV) regimens. We investigated whether specific psychiatric disorders, illicit drug use, and levels of alcohol use were associated with ARV adherence in a national sample of adults receiving care for HIV. METHODS: The HIV Cost and Services Utilization Study interviewed a population-based multi-stage probability sample of adults with HIV within the U.S. Data on mental health and substance use were collected using the short form composite international diagnostic interview (CIDI-SF) with supplemental substance use and alcohol use questions. Those interviewed in $1998(\mathrm{~N}=2267$, representing 181,557 in care) included $\mathrm{N}=1910$ patients taking ARVs, who answered self-report questions about medication adherence during the prior week. RESULTS: Fifty-three percent of HIV patients using ARVs reported some nonadherence to these medications. Psychiatric disorders (19\%), illicit drug use (28\%), and heavy alcohol use (14\%) were all common. In multivariable regression models adjusting for gender, ethnicity, age, education, insurance, CD4 cell count, AIDS diagnosis, and type of antiretroviral therapy, HIV patients with depression [odds ratio (OR) 1.7, 95\% Confidence Interval (CI) 1.3-2.3], generalized anxiety disorder [OR 2.4, 95\% CI 1.2-5.0], and panic disorder [OR 2.0, 95\% CI 1.4-3.0] were more likely to be nonadherent to ARVs than those without psychiatric disorders.
Use of cocaine [OR 2.2, 95\% CI 1.2-3.8], marijuana [OR 1.7, 95\% CI 1.2-2.3], or and amphetamines [OR $2.3,95 \%$ CI $1.2-4.2$ ] during the prior month were each associated with ARV nonadherence. Compared to those who did not use any alcohol, ARV nonadherence was more common among moderate [OR 1.6, 95\% CI 1.3-2.0] and heavy [OR 1.7, 95\% CI $1.3-$ $2.3]$ drinkers, and and was most common [OR 2.7, 95\% CI 1.7-4.5] among frequent-heavy drinkers of alcohol.

CONCLUSION: HIV patients with mental health problems and illicit substance use reported significantly more nonadherence to ARV medications. The likelihood of nonadherence increased with progressively higher levels of alcohol use. Clinical programs to effectively screen for and treat mental health, illicit drug use, and alcohol use problems may be needed in HIV patients to achieve good ARV adherence, and therefore optimal HIV suppression and health outcomes.

QUALITY OF LIFE IN SYNCOPAL PATIENTS. N. Van Dijk ${ }^{1}$, M. Sprangers ${ }^{2}$, W. Wieling ${ }^{1}$ M. Linzer ${ }^{3}$; ${ }^{1}$ Academic Medical Center, Department of Internal Medicine, Amsterdam, Amsterdam, Netherlands; ${ }^{2}$ Academic Medical Center, Department of Medical Psychology, Amsterdam, Amsterdam, Netherlands; ${ }^{3}$ University of Wisconsin-Madison, Madison, WI (Tracking ID \#73871)

BACKGROUND: Sudden loss of consciousness may be physically and socially incapacitating. Uncertainty about the cause and fear of an episode occurring in a crowded or dangerous situation can influence social function. Only a few small studies have assessed quality of life (QOL) in patients with syncope. We sought to determine QOL-issues in a large cohort of syncope patients from emergency departments and referral sources.

METHODS: As a part of the Dutch Fainting Assessment Trial (FAST) we asked 486 consecutive patients presenting to all departments of the Academic Medical Center with loss of consciousness to complete: 1) the SF-36, a generic quality of life questionnaire, and 2) the Syncope Dysfunction Scale (SDS), a previously validated disease specific questionnaire which assesses specific areas of impairment due to syncope, and fear and worry about syncopal spells. RESULTS: Response rate to the questionnaires was $81 \%(n=395)$. Measured by the SF-36, quality of life (QOL) in patients with syncope is moderately to seriously impaired when compared to the normal Dutch population (e.g. scored from $0-100 ; 100=$ perfect health: physical functioning 68 in syncope patients vs 85 in normals $(\mathrm{p}<.01)$; social functioning 67 in syncope patients vs 84 in normals $(\mathrm{p}<.01)$. Disease specific impairment was moderate, with an impairment frequency of $21-51 \%$ of the 11 listed activities. QOL in women with syncope was significantly more impaired than in men in the areas of physical functioning, bodily pain, general health and vitality. A clear correlation was found between the number of episodes in the last 12 months and almost all QOL domains.

CONCLUSION: QOL is seriously affected in patients with syncope, moreso in women than men. The number of episodes in the year before presentation predicts lower QOL. The impaired QOL in this group of patients justifies an intensive approach to diagnosis and treatment.

LONG QT SYNDROME AND OBSTRUCTIVE SLEEP APNEA. N. Volkova ${ }^{1}$, J.C. Luck ${ }^{1}$, E.O. Bixler ${ }^{1} ;{ }^{1}$ Penn State Milton S. Hershey Medical Center/Penn State University College of Medicine, Hershey, PA (Tracking ID \#73591)

BACKGROUND: Patients with obstructive sleep apnea (OSA) are considered to be at increased risk for sudden cardiac death. The long QT syndrome (LQTS) is associated with polymorphic ventricular tachycardia and is a known cause of sudden cardiac death. The present study was undertaken to determine if there is an association between OSA and the LQTS. METHODS: We conducted this case-control study based on a random sample of the general public and a group of patients from the Sleep disorders clinic.

A total of 633 subjects were selected for this study. The first group included 140 consecutive patients evaluated in a Sleep disorders clinic for OSA (clinic OSA) Second group included 87 patients with OSA from the random sample of the general population (general population OSA). Both of these groups required CPAP treatment. The control group included 400 subjects without OSA. They were selected from the same random sample and matched by age and body mass index (BMI) to the clinic OSA group.

The QT interval was measured manually and the corrected QT (QTc) was calculated (Bazett formula). LQTS was considered if QTc was greater than 0.45 seconds for males and 0.47 seconds for females. Odds ratio (OR), Logistic Regression and ANOVA (controlling for age and BMI) were used for statistical analysis.

RESULTS: OR of LQTS in the clinic OSA versus the control group was 7.4 (3.0,18.5), $\mathrm{P}<0.001$ and in the general population OSA versus the control group was 4.1 $(1.4,12.6), \mathrm{P}=0.02$. Using Logistic Regression to control for age and BMI for clinic and general population OSA, OR was 2.6 and $2.3, \mathrm{P}=0.11$ and 0.002 respectively. The mean QTc intervals for the clinic OSA and the general population OSA were $0.430 \pm 0.002$ and $0.412 \pm 0.003$ seconds respectively, and were significantly higher compared to the control group $0.403 \pm 0.001$ (both $\mathrm{P}<0.05$ ).

CONCLUSION: The prevalence of LQTS in patients with OSA was significantly greater than in control subjects. An increase in the sudden cardiac death in patients with OSA might be related to a LQTS leading to ventricular tachyarrythmias. In the OSA patients an electrocardiogram should be performed to screen for LQTS and special precautions need to be taken with medications that may prolong the QTc interval. This simple modality of avoiding these medications could be lifesaving for some OSA patients.

FACTORS ASSOCIATED WITH READINESS TO LOSE WEIGHT AMONG PRIMARY CARE PATIENTS. C.C. Wee ${ }^{1}$, R.B. Davis ${ }^{1}$, R.S. Phillips ${ }^{1} ;{ }^{1}$ Division of Gen. Medicine and Primary Care, Beth Israel Deaconess Medical Center, Harvard Medical School, Boston, MA (Tracking ID \#73887)

BACKGROUND: While almost two-thirds of Americans are overweight (BMI $>25 \mathrm{~kg} / \mathrm{m} 2$ ), a large proportion of patients (pts) are not "ready" for action-oriented weight (wt) loss 
interventions. We describe factors associated with being in the preparation (prep) or action stages for wt loss.

METHODS: We surveyed via telephone a random sample of primary care pts at an academic medical center. We collected demographic and clinical factors (e.g. comorbid illness, height, wt, wt history), provider (MD) counseling about wt, and pts' preceptions of health, wt, and their stage of wt change. We used bivariable and logistic regression analyses to identify correlates of being in the prep (thinking about losing wt in the next month) or action (trying to lose wt now) stages of wt change.

RESULTS: Of 216 pts surveyed to date (61\% response rate), the mean age was 49 years, $62 \%$ were white, and $35 \%$ were men; $56 \%$ were in the prep or action stages of wt change. Of all pts, $64 \%$ were overweight, but only $38 \%$ thought their wt was a health risk and only $27 \%$ were ever advised by their MD that their wt may be a health risk. Before adjustment, factors significantly associated with being in the prep or action stages included higher BMI, being black or hispanic ( $69 \%$ vs. $55 \%$ in whites), having lost wt in the past ( $73 \%$ vs. $26 \%$ ), believing one's wt was a health risk $(84 \%$ vs. $39 \%)$, and MD counseling about wt $(83 \%$ vs. $47 \%)$. After adjusting for age, sex, education and wt-related illness, being overweight [odds ratio (OR) 4.9, (95\% CI 1.9-12.6)] compared to normal wt, having lost wt in the past [OR $6.0(2.6-13.8)$ ], and believing one's wt was a health risk [OR 8.1 $(3.0-22.1)]$ were associated with being in the prep or action stages. Pts were more than 4 times as likely to believe that their wt was a health risk if they were told so by their providers in the past [RR $4.4(3.2-6.1)]$.

CONCLUSION: Pts' readiness to undergo wt control interventions is strongly associated with the belief that their weight is a health risk. This belief, in turn, is highly correlated with provider counseling about the risk of wt to health. Primary care providers should improve efforts to address the health risk of being overweight with their pts.

THE EPIDEMIOLOGY OF VENOUS THROMBOEMBOLISM IN CALIFORNIA IN 1996 R.H. White ${ }^{1}$, P.S. Romano ${ }^{1}$; ${ }^{1}$ University of California, Davis, Sacramento, CA (Tracking ID \#74532)

BACKGROUND: There have been few studies of the epidemiology of venous thromboembolism (VTE) in ethnically diverse populations.

METHODS: We used the linked California Patient Discharge Data Set to identify a cohort that was hospitalized and coded as having VTE in 1996. Cases that had a prior diagnosis of VTE at any time back to July 1, 1990 were excluded. The remaining cases were classified as probable-VTE or possible-VTE based on the strength of coding criteria. Cases coded for both deep-vein thrombosis (DVT) and pulmonary embolism (PE) were classified as having PE. Incidence rates were directly standardized to the 1996 population in California

RESULTS: There were 16,417 cases identified with probable VTE and an additional 6,063 had possible VTE. The overall annual incidence of probable VTE was 70 per 100,000 adults age 18 or over; 63 in men, 78 in women, 86 in Caucasians, 93 in African- Americans, 37 in Latinos and 19 in Asian-Pacific Islanders. The incidence rose from 7/100,000 for persons $18-24$ years old to $362 / 100,000$ among cases over age 85 . The relative proportion of PE to total VTE cases was significantly lower among Latinos (25.8\%) and Asian-Pacific Islanders $(27.9 \%)$ compared to Caucasian $(31.5 \%)$ or African Americans $(32.9 \%)(\mathrm{p}<0.001$ all comparisons). Underlying risk factors were: $18 \%$ with cancer, $2 \%$ with trauma $<2$ months, $20 \%$ with surgery $<2$ months, $2 \%$ with recent hospitalization of 4 or more days within 2 weeks, $7 \%$ concurrent medical hospitalization, and $52 \%$ had 'idiopathic' VTE.Cardiopulmonary disease was present in $40 \%$ of PE cases compared with $27 \%$ of DVT cases $(\mathrm{p}<0.001)$. Among the 8448 cases classified as idiopathic VTE, $2.1 \%$ were re-hospitalized within 6 months with a new diagnosis of cancer. The 6-month cumulative incidence of recurrent VTE was $4.8 \%$, with an incidence of $5.9 \%$ among cancer cases, $3.5 \%$ among postsurgery cases, and $5.3 \%$ among idiopathic cases. The 6-month recurrence rate after idiopathic PE and DVT were similar, 5.3\% each.

CONCLUSION: The incidence of VTE, and particularly PE, is significantly lower among Latinos and Asian-Pacific Islanders who live in California.The annual incidence rates for VTE among Caucasians and African-Americans that we calculated using administrative data are similar to findings from other population-based studies.

EFFECT OF AGE ON THE INCIDENCE OF VENOUS THROMBOEMBOLISM AFTER SURGERY. R.H. White ${ }^{1}$, B. Gage ${ }^{2}$, P.S. Romano'; ' ${ }^{1}$ University of California, Davis, Sacramento, CA; ${ }^{2}$ Washington University in St. Louis, St. Louis, MO (Tracking ID \#74628) BACKGROUND: Guidelines for use of thromboprophylaxis after major surgery use advancing age (e.g. age $<40,40$ to 60 , or age $>60$ ) to stratify the risk of post-operative venous thromboembolism (VTE). We sought to better characterize the effect of age on postsurgery VTE by analyzing the incidence of symptomatic VTE after a spectrum of surgical procedures.

METHODS: Using the California Patient Discharge Data Set, a large linked database, we determined the 3-month incidence of VTE, defined using specific ICD-9-CM codes, after selected surgeries, defined by procedure code(s). Logistic regression was use to model the effect of age (in 5-year increments), race/ethnicity, sex, presence of comorbidities, and the surgical procedure on development of VTE.

RESULTS: 1,464,452 cases underwent one of 41 different procedures (mean cases per procedure $=35,718)$. In logistic models there was a strong, statistically significant, interaction between age and the type of surgery performed $(\mathrm{p}<0.0001)$. Qualitative analysis of the effect of age on the incidence of VTE revealed three general patterns: 1) increase in the incidence of VTE with age, 2) an increase in VTE up to age $~ 65$ with no increase thereafter, and 3) no change in VTE with age.

CONCLUSION: The relationship between age and the risk of VTE after surgery is complex and depends on the specific procedure.

\section{- Partial or Complete Coledomy, no cancer; -VTE rises with age -... Total Hip A.rthroplasty -VTE rises only to mean age 65 Peripheral Vasoular Shunt or Bypass - no change (or fall) in VTE with age}

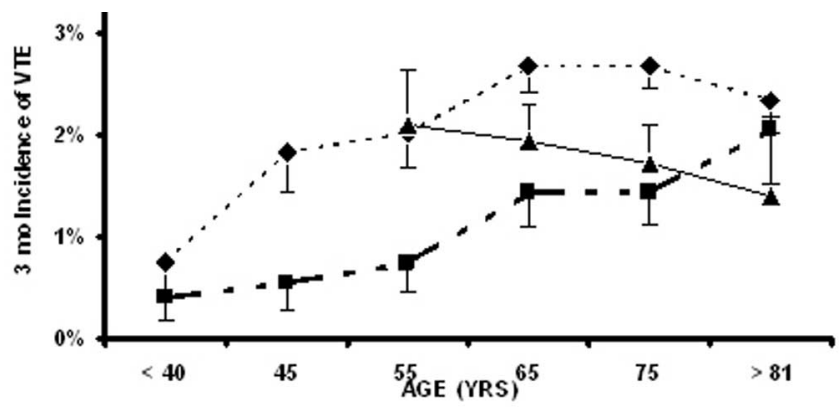

Figure 2

ANTICOAGULATION IN A RESIDENT CLINIC - NURSE OR RESIDENT MANAGED? K.C. Wilson ${ }^{1}$, S. Rusch ${ }^{1}$, T. Foster ${ }^{1}$; ${ }^{1}$ University of Illinois at Peoria, Peoria, IL (Tracking ID \#76002)

BACKGROUND: Many published studies have demonstrated the efficacy of a standardized protocol for warfarin dosing but none have compared usual resident management (URM) to nurse managed protocol (NMP) in an indigent care Internal Medicine resident clinic.

METHODS: From 11/1/01 to 1/31/02, all INRs on 67 URM patients were reviewed. During the following 5 months, all eligible patients were enrolled in the NMP. From 6/1/02 to 8/31/02, all INRs on $50 \mathrm{NMP}$ patients were reviewed. INRs were considered therapeutic (INR 2-3), near therapeutic (INR 1.8-3.3), nontherapeutic $(<1.8$ or $>3.3)$, or high risk (INR $>5$ ). RESULTS: Comparing URM patients to NMP patients, $8 / 67$ (11.9\%) vs. $1 / 50$ (2\%) were therapeutic every time measured over the three month period, $15 / 67(22.4 \%)$ vs. $5 / 50(10 \%)$ were near therapeutic each time measured. 117 patients had a total of 233 nontherapeutic INRs, out of 630 total INRs. Comparing URM to NMP, 13/365 (3.6\%) vs. 5/265 (1.9\%) were high risk.

CONCLUSION: A nurse managed anticoagulation program in an internal medicine residency clinic decreased the number of high risk INRs but did not increase the number of patients maintained at a therapeutic INR. In both scenarios, patients in this population were poorly anticoagulated.

TAI CHI AS AN ADJUNCTIVE INTERVENTION FOR PATIENTS WITH HEART FAILURE: A PILOT STUDY. G.Y. Yeh ${ }^{1}$, D.M. Eisenberg ${ }^{1}$, M.J. Wood ${ }^{2}$, P.M. Wayne ${ }^{3}$, B.H. Lorell ${ }^{4}$ L.W. Stevenson ${ }^{5}$, A.L. Goldberger ${ }^{4}$, R.B. Davis ${ }^{4}$, R.S. Phillips ${ }^{4} ;{ }^{1}$ Harvard Medical School, Boston, MA; ${ }^{2}$ Massachusetts General Hospital, Boston, MA; ${ }^{3} \mathrm{New}$ England School of Acupuncture, Boston, MA; ${ }^{4}$ Beth Israel Deaconess Medical Center, Boston, MA; ${ }^{5}$ Brigham and Women's Hospital, Boston, MA (Tracking ID \#73851)

BACKGROUND: Exercise training is an accepted component of therapy in patients with chronic heart failure. Aside from conventional cardiac rehabilitation programs, little is known about the potential benefit of mind-body movement therapies such as Tai Chi in this population. OBJECTIVE: To investigate whether Tai Chi may be a beneficial adjunct to standard treatment in patients with chronic heart failure and to determine whether a randomized controlled trial is feasible.

METHODS: We randomized nineteen patients with chronic stable heart failure, systolic dysfunction, and ejection fraction of $40 \%$ or less to receive either usual care plus twelve weeks of supervised Tai Chi training $(n=10)$ or usual care alone as a wait-list control $(n=9)$. For all patients, usual care included pharmacologic therapy and diet counseling as described in current ACC/AHA Consensus Guidelines. The Tai Chi intervention consisted of a 1-hour class twice weekly and a video for home practice. Outcomes measured at baseline and at 12 weeks included quality of life using the Minnesota Living with Heart Failure Questionnaire (MLHF, range 0$105,0=$ best $)$ and exercise capacity using the six-minute walk test.

RESULTS: The mean age of study patients was $62.8 \pm 3.7$ years, mean baseline ejection fraction was $23 \pm 1.9 \%$, median New York Heart Association Class was 2, and $58 \%$ were male. There were no significant differences between intervention and control patients at baseline, including MLHF scores and six-minute walk distance. The most common causes of heart failure were idiopathic dilated cardiomyopathy ( $42 \%)$ and ischemic cardiomyopathy $(26 \%)$. Nearly $90 \%$ of patients were taking ACE inhibitors and $84 \%$ of patients were on beta-blockers. Patients in the intervention group improved scores on the MLHF, while control patients had worsened scores at 12 weeks (change of $-16.1 \pm 4.3$ vs. $10.7 \pm 5.9$ points, $p<.01$ ). Similarly, patients in the intervention group increased their six-minute walk distance, while control patients decreased this distance (percent change of $27 \pm 4.5 \%$ vs. $-21 \pm 12.5 \%, p<.01$ ). CONCLUSION: Mind-body movement therapies such as Tai Chi may be a useful adjunct to standard care in patients with chronic heart failure and warrant further investigation.

'WHEN SICKENING GRIEF DOTH PREY': THE ASSOCIATION OF DEPRESSION AND MORTALITY IN CANCER PATIENTS. S. Zickmund ${ }^{1}$, J. Johnson ${ }^{1}$, A. Christensen ${ }^{1}$; ${ }^{1}$ University of lowa, lowa City, IA (Tracking ID \#76820)

BACKGROUND: Chronic, potentially terminal physical diseases carry a significant emotional burden. Recent studies suggest that depression is an independent predictor of poor outcome in 
diseases such as advanced heart failure. However, the literature remains inconclusive on the effect of depression on patients with malignancies. We hypothesized that depression was similarly associated with a higher likelihood of dying in patients with cancer.

METHODS: Patients with a diagnosis of cancer treated at the University of Iowa Cancer Center were recruited between October 1999 and August 2002. Participants provided demographic information and completed the Hospital Anxiety and Depression Scale (HAD). Site and stage of malignancy at the time of diagnosis were obtained. All patients were followed for survival. Predictors of patient survival were determined using a Cox regression analysis. RESULTS: Of the 491 enrolled patients $(60 \%$ women, age 56.4$)$, the main diagnoses were neoplasms of the gastrointestinal tract (15\%), lung and upper airways (10\%), breast (14\%), ovaries $(18 \%)$, lymphoma (12\%) or hematological malignancies $(14 \%)$. During the average follow up of $15.9(0.1-42)$ months, $44 \%$ of the patients died. In addition to the expected predictors age $(\mathrm{p}<0.01)$ and disease stage $(\mathrm{p}<0.0001)$, only the depression score $(\mathrm{p}<0.01$, hazard ratio 1.074 , mean 4.7, SD 3.5) was an independent predictor of death. Thus every standard deviation increase on the depression scale is associated with a $25.7 \%$ increase in the hazard of death. In contrast to depression, the anxiety score ( $\mathrm{p}<0.178$, mean 6.6, SD 3.6) of the HAD did not correlate with the likelihood of death within the time period studied.

CONCLUSION: This study found that high scores on the depression scale were an independent predictor of mortality. These results obtained in a cross-sectional study suggest that health care providers should screen their patients for depression and consider treatment. Prospective, randomized studies are needed to determine whether such interventions will affect both quality and duration of life. 